University of Nebraska - Lincoln

DigitalCommons@University of Nebraska - Lincoln

MANTER: Journal of Parasite Biodiversity

Parasitology, Harold W. Manter Laboratory of

10-15-2021

Natterer in Neotropical Nematoda: Species Described by

Rudolphi, Diesing, and Molin

Ricardo Guerrero

Universidad Central de Venezuela, Caracas, ricardo.guerero.ucv@gmail.com

Follow this and additional works at: https://digitalcommons.unl.edu/manter

Part of the Biodiversity Commons, Parasitology Commons, and the Zoology Commons

Guerrero, Ricardo, "Natterer in Neotropical Nematoda: Species Described by Rudolphi, Diesing, and Molin" (2021). MANTER: Journal of Parasite Biodiversity. 18.

https://digitalcommons.unl.edu/manter/18

This Article is brought to you for free and open access by the Parasitology, Harold W. Manter Laboratory of at DigitalCommons@University of Nebraska - Lincoln. It has been accepted for inclusion in MANTER: Journal of Parasite Biodiversity by an authorized administrator of DigitalCommons@University of Nebraska - Lincoln. 


\title{
Natterer in Neotropical Nematoda: Species Described by Rudolphi, Diesing, and Molin
}

\author{
Ricardo Guerrero
}

Laboratorio de Biología de Vectores y Parásitos, Instituto de Zoología y Ecología Tropical, Fac. de Ciencias, Universidad Central de Venezuela, Caracas, Venezuela (ricardo.guerero.ucv@gmail.com) ORCID: 0000-0003-0925-9476

\begin{abstract}
Between 1817 and 1835 Johann Natterer collected 1,729 samples of endoparasitic helminths in Brazil and southern Venezuela. Of the 323 names that were assigned to the collected nematode specimens, 163 are still valid and accepted species, 84 are still doubtful, and 76 must be rejected. In this work, each name is analyzed and correlated to the literature to clarify its taxonomic status.
\end{abstract}

\section{Introduction}

Johann Natterer was born in Laxenburg near Vienna on 9 November 1787. His father was an imperial falconer and collector of birds and insects and taught him how to hunt and preserve animals to be museum specimens. He had the opportunity to make excursions in the regions of Banato (Hungary) and Sclavonia (Croatia), whereupon he returned to Vienna in 1810. Later he worked diligently on helminthology, traveling from 1813 to 1814 at his own expense to Italy as far as Calabria and repeatedly examining the biology of the coastline on the Adriatic Sea. In 1816 he was employed by the Imperial Museum of Natural History of Vienna and in 1817 was a member of an expedition-together with J.B.E. Pohl, J.B.R. von Spix, K.F.P. Martius, and others-to Brazil to collect and study the animals and plants of the country.

From Chioggia in Italy the frigate Augusta sailed to Gibraltar on 31 May 1817 and then awaited the arrival of the Imperial Archduchess Bride on the Portuguese liner Dom Joã until 1 September, giving Natterer the opportunity to explore the southern tip of Spain. On the way to Brazil, a short expedition was launched in Funchal, the capital of the island of Madeira, but only one and a half days were spent on exploration. On 5 November the Augusta finally arrived at Ilha das Cobras in the magnificent bay of Rio de Janeiro.
From 1817 to 1835 Natterer mounted 10 separate scientific collecting expeditions into the interior of Brazil, starting from Rio de Janeiro and going to Mato Grosso and then into the Amazon basin. While in the Amazon ba$\sin$, he descended the Rio Madeira to Manaus, where he married. Later, he led an expedition upstream on the Rio Negro to the border of Colombia and then on to San Carlos de Rio Negro in Venezuela and later up the Rio Branco to Fort do São Joaquim near present-day Boa Vista. In 1835 he collected specimens in Pará to the mouth of the Amazon, where he was surprised by a revolution and lost some of his collections. Finally, Natterer boarded a British government ship to Europe on 15 September 1835 and returned to Vienna via London in 1836-an eighteen-year absence (Goeldi, 1896; Papavero, 1971; Hershkovitz, 1987; Vanzolini, 1993).

During his travels, Natterer collected many samples of animals, eggs, seeds, woods, ethnographic objects, and 1,729 vials of endoparasites, which were deposited in $\mathrm{Vi}$ enna (Fitzinger, 1833, 1868; Schröckinger-Neudenberg, 1855; Schmutzer, 2011). However, several samples collected during the first part of his expeditions in Brazil are in Berlin (Hartwich, 1964b, 1964c, 1965, 1966, 1970, 1971, 1972).

The species of Nemata or Nematoda collected by him were described by Rudolphi (1819), Diesing $(1851,1857)$, Molin (1858a, 1858b, 1858c, 1859a, 1859b, 1860a, 1860b, 
1860c, 1860d, 1860e, 1861a, 1861b), Schneider (1866), and Drasche (1882a, 1882b). The descriptions are in the main very poorly done, composed of only a few lines and usually without figures or drawings to support the study. The purpose of this review of the material collected by Natterer is to establish a complete list of all described species; their current status; and whether they are valid species, synonyms, or nomen nudum as well as to update the hosts and the original dates and localities where they were collected.

\section{Materials and Methods}

To prepare the list, I reviewed all publications of C. A. Rudolphi, K. M. Diesing, R. Molin, A. F. Schneider, and R. von Drasche in which exemplars collected by Natterer were used. In the list are the names as they appear in those publications along with the reference in which the species is first described. I also included all publications in which Natterer appears and other publications relevant to the species. To complement the data on the hosts, localities, and dates, I used the books of Pelzeln $(1871,1883)$.

\section{Results}

Filaria abbreviata Rudolphi, 1819

Filaria abbreviata, Rudolphi, 1819: 4, 210.

Filaria abbreviata, Dujardin, 1845: 52.

Filaria abbreviata, Diesing, 1851: 268.

Filaria abbreviata, Molin, 1858b: 396.

Filaria abbreviata, Stossich, 1898: 57.

Diplotriaena abbreviata (Rudolphi, 1819), Railliet \& Henry, 1909c: 547.

Material: "Specimina tria mecum communicavit circa oculum Moteicillae stapazinae (Oenanthe hispanica) Bremser" (Rudolphi). M. (Motacilla) sp. 144 (Rhynchocyclus olivaceus), in abdomine, Brasilia (Diesing); Sturnus pyrrbocephalus (sic) (Amblyramphus holocericeus) $6 \hat{\jmath} \widehat{\jmath}, 9$ qq, Caiçara, Octobri and Junio, in eorum cavo abdom.; Tanagra jacapa (Icterus cayenensis) 1 q, Rio das Pedras (Riberao das Pedras), Junio, sub cuticula ad renem dextrum; Thryothorus polyglottus (Mimus saturninus) 1 , Vendinha apud Pirapitingui, Novembri, in cavo abdom.; Furnarius rufus, 10 , 5 우, Cuyaba, Junio, in cavo abdom., same host and locality, Januario, Majo, Julio, ad renes; Furnarius leucopus, 1 d̂, 1 q, Cuyaba, Junio, in eorum cavo abdom. (Molin).

Remarks: Rudolphi (1819: 4) named Filaria abbreviata from around the eye cavity of Motacilla stapazina with a sample in MCV without any description but under "mouth simple species." In the same paper (p. 210),
Filaria abbreviata $\mathrm{n}$. sp. is described from the same host and from males and females in the nose and eyes of Falco naevii (Accipter gentilis). If collected by Bremser, the host is European.

Dujardin (1845) gave the total length of males and females of both hosts along with a short description and pointed out that in the catalogue of Vienna samples those from Falco naevii are under the name Spiroptera stereura.

Diesing (1851) included Filaria turdorum Rudolphi, 1819; Filaria motacillarum Rudolphi, 1819; and Filaria motacillae Rudolphi, 1819 as synonyms of F. abbreviata without any commentary and gathered under this name all the material studied by Rudolphi, including the material from the abdomen of M. 144 collected by Natterer in Brazil, placing the material of Motacilla melanocephala (stapazina of Rudolphi) as collected in the abdomen.

Molin (1858b) repeats Diesing's synonyms and the error of placing M. melanocephala's material as collected in the abdomen, noting that it was not found, and also includes other material collected by Natterer in Brazil. The description includes a 6-tooth armed mouth.

Stossich (1898) places the species under the group of "Forme Inquirende" and repeats the synonyms, including F. philomelae Diesing, 1851, and also repeats Molin's description that assigns all the material as being from the body's cavity.

Based on these descriptions, Railliet and Henry (1909c) include this species, with its synonyms, in the genus Diplotriaena, criterion followed by Yamaguti (1961). However, Anderson (1958) in his review of the genus points out "the species was originally described from the orbit of Motacilla stapazina, and samples probably refer to various species of Diplotriaena, but inasmuch as little, or no, description accompanies them, so too should this be regarded as nomina nuda."

In conclusion, as F. abbreviata has been assigned material of two or more host species, the original from the orbital cavity of Motacilla stapazina that could be a species of Aprocta and the material originally described as Filaria motacillae from the abdomen of Muscicapae n. 144, which appears to be a kind of Diplotriaena, but until the original material is reviewed, if this exists, both must remain as nomen dubium, or if the material of $M$. stapazina disappeared, as pointed out by Molin (1858b), Filaria abbreviata would be nomen nudum and Filaria motacillae would become Diplotriaena motacillae.

Status: Nomem nudum.

Hystrichis acanthocephalicus Molin, 1861

Hystrichis acanthocephalicus, Molin, 1861a: 608, Tab. IX.

Material: Ibis nudifrons (Phimosus infuscatus), 5 o, 16 
१, Caiçara, 11-xi-1825, inter tunicas proventriculi; $1 \AA, 1$, same host and locality, 29-v-1826.

Status: Hystrichis acantocephalicus Molin, 1861.

\section{Ascaris acuminata Schrank, 1788}

Ascaris acuminata, Schrank, 1788: 12.

Ascaris acuminata, Diesing, 1851: 152.

Aplectana acuminata, Railliet \& Henry, 1916: 249.

Material: Phrynohyas tschudii (Trachycephalus sp. ?), Januario; Calamites luteus (Hyla sp. ?), Octobri; Docidophryne agua (Rhinella icterica); and Leptodactylus sibilatrix (Leptodactylus fuscus), Novembri et Decembri, in eorum intestinis praesertim crassis.

Remarks: Aplectana acuminata is restricted to Western Europe (Baker, 1980), and several species have been recorded in South America.

Status: Until review of the exemplars used by Diesing, the species will remain as species inquirenda.

\section{Spiroptera acuminata Molin, 1860}

Spiroptera acuminata, Molin, 1860a: 930.

Spiroptera acuminata, Drasche, 1883b: 195, Taf. XII, fig.

18 und 19; Taf. XIV, fig. 18.

Oxyspirura acuminata, Stossich, 1898: 123.

Rhabdochona acuminata, Railliet, 1916: 105.

Rhabdochona (Filochona) acuminata, Saidov, 1953: 627.

Filochona acuminata (Molin, 1960), Skrjabin et al., 1967: 363.

Material: Brycon falcatus (Acestrorhynchus falcatus), 4 ôd, Matogrosso, 10-11-1826, in intestino.

Status: Rhabdochona acuminata (Molin, 1859) Railliet, 1916.

Filaria acuticauda Molin, 1858

Filaria acuticauda, Molin, 1858b: 379.

Filaria acuticauda, Stossich, 1898: 58.

Material: Dasypus loricatus (Cabassous unicinctus), 3 ôd, 6 웅, Ypanema, Novembri, inter glandulas colli; Dasypus niger (Dasypus novemcinctus), 1 , Ypanema, Februario, sub cute colli; $1 \hat{0}, 1$, same host and locality, Decembri, ad faciem externam oesophagi.

Status: At the moment, the species is still unredescribed. Probably these are two different species, one Dipetalonema and the other Achantocheilonema; if so, then it is incertae sedis.

\section{Physaloptera acuticauda Molin, 1860}

Physaloptera alata, Diesing, 1851: 234 (in partim).

Physaloptera acuticauda, Molin, 1860d: 661.

Physaloptera acuticaudal, Drasche, 1882a: 127, Taf.
VIII, fig. 4 und 5.

Physaloptera acuticauda, Ortlepp, 1922: 1050, fig. 26.

Material: Falco cachinans (Herpetotheres cachinnans),

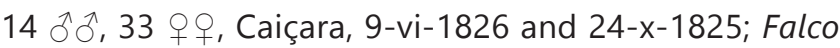
gracilis (Geranospiza coerulescens), 1 J, 7 q, , Marabita-

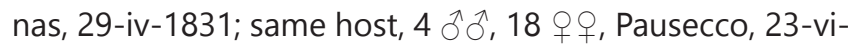
1826; Falco swainsonii (Gampsonyx swainsonii), 2 ôे $\hat{\alpha}$, Esliva, 4-vii-1826; Falco rutilans (Heterospizias meridionales), 1 , Cuyaba, 30-iv-1824; Falco ornatus (Spizaetus ornatus), 4 ถิ $\hat{\partial}$, Ypanema, 27-ix-1819; same host, 6 ô $\hat{0}, 6$ 웅, Rio Branco apud Serra Caruman, 8-vi-1832; Falco palustris (Circus buffoni), 1 0,3 + $q$, Cuyaba, 30-iv-1824; Falco coronatus (Harpyhaliaetus coronatus), 5 숭, 9 우, Rio Araguay, 31-x-1823, same host Rio Araguay, same host, 1 + , Borba, 2-viii-1830; Falco urubutinga (Buteogallus urubutinga), 1 , Egenho do Pari, 21-vi-1825; Falco dispar (Elanus leucurus), 1 $\hat{0}, 1$, Irisanga, Februario; Falco atricapillus (Spizastur melanoleucus),

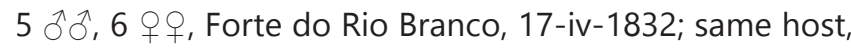
$2 \widehat{\jmath} \widehat{0}, 2$ 우우, Ypanema, 21-x-1821; Falco cayennensis (sic) (Leptodon cayanensis), 3 के $\hat{0}, 2$ + + ; Falco unicinctus (Parabuteo unicinctus), 18 ๙ึ่ 25 우우, Matogrosso, 29-vi-1827 and 6-vii-1828; Falco minutus (Buteo brachyurus), 1 ภ, 1 의, Mattogrosso, 1-vii-1828, in oesophago et ventriculo.

Status: Physaloptera acuticauda Molin, 1860.

Subulura acutissima Molin, 1860

Subulura acutissima, Molin, 1860d: 332.

Subulura acutissima, Diesing, 1861: 644.

Subulura acutissima, Drasche, 1882a: 120, Taf. VII, fig. 11-14.

Material: Strix atricapilla (Megascops atricapilla), Augusto; Cuculus melacoryphus (Coccyzus melacoryphus), in both hosts $4 \hat{0} \hat{D}^{2}, 42$ 우, in ventriculo et in intestinis.

Status: Subulura acutissima Molin, 1860.

Filaria acutiuscula Molin, 1858

Filaria acutiuscula, Molin, 1858b: 388.

Dirofilaria acutiuscula, Chitwood, 1933: 63.

Dirofilaria acutiuscula, Vicente et al., 1998: 169.

Material: Canis azarae (Cerdocyon thous), Barra do Rio Jauru, Octobri, ad costas sub musculis pectoralibus; Dicotyles albirostris (Tayassu pecari), $3 \hat{\circ} \partial^{\lambda}, 11+q$, Matogrosso, Novembri, in cistibus inter laminas paritonei et inter tunicas ventriculi; Dicotyles torquatus (Pecari tajacu), Caiçara, 29-ii-1826, in mesenterio.

Status: Dirofilaria acutiuscula (Molin, 1858) Chitwood, 1933.

Filaria aequalis Molin, 1858

Filaria aequalis, Molin, 1858b: 383.

Solenonema aequale, Diesing, 1861: 704. 
Material: Myrmecophaga jubata (Myrmecophaga tridactyla), 2 $\hat{\jmath}, 4$ 웅, in Brasilia.

Remarks: Filaria aequale was moved to the genus Solenonema by Diesing together with two other species; the genus was characterized by "penis vagina tubulosa vel subglobulosa" (Diesing, 1861: 620). These species are from three different types of hosts: Pilosa, Chiroptera, and Carnivora. The genus has gone unnoticed or considered by most of the authors: Filaria sensu lato (Baylis and Daubney, 1926), "Filarioidea insufficiently known" (Yorke \& Maplestone, 1926; Yamaguti, 1961), or close to Onchocercella (Chabaud, 1965).

Status: No other species of Filaria has subsequently been reported from that host in Brasil (Vicente et al., 1998), so until the original material is reviewed, it should be considered as species inquirenda.

Trichocephalus affinis Rudolphi, 1802

Trichocephalus ovis, Abildgaard, 1795: 37.

Trichocephalus affinis, Rudolphi, 1802: 7.

Trichocephalus cameli, Rudolphi, 1819: 18.

Trichocephalus affinis, Diesing, 1851: 290.

Trichuris ovis, Smith, 1908: 269.

Material: Cervus dichotomus (Blastocerus dichotomus), Novembri; Cervus simplicicornis (Mazama gouazoubira), Julio.

Status: Trichuris ovis (Abildgaard, 1795) Smith, 1908.

\section{Physaloptera alata Rudolphi, 1819}

Physaloptera alata, Rudolphi, 1819: 256.

Physaloptera megalostoma, Creplin, 1829: 6.

Spiroptera physalura, Dujardin, 1845: 94.

Spiroptera megalostoma, Dujardin, 1845: 96.

Physaloptera alata, Diesing, 1851: 234.

Physaloptera alata, Ortlepp, 1922: 1025.

Material: Falco cachinans (Herpetotheres cachinnans), Junio, in oesophago; same host, Octobri, in ventriculo; Falco gracilis (Geranospiza coerulescens), Aprili et Junio, in oesophago et ventriculo; Falco swainsonii (Gampsonyx swainsonii), Julio, in oesophago; Falco rutilans (Heterospizias meridionales), Aprili, in ventriculo; Falco ornatus (Spizaetus ornatus), Junio, in oesophago et ventriculo; Falco palustris (Circus buffoni), Aprili, in oesophago et ventriculo; Falco coronatus (Harpyhaliaetus coronatus), Octobri, in cavo orbitae, in regione occipitali et in faucibus; Falco urubutinga (Buteogallus urubutinga), Junio, in ventriculo; Falco dispar (Elanus leucurus), Februario, in ventriculo; Falco atricapillus (Spizastur melanoleucus), Aprili et Octobri, in oesophago et ventriculo; Falco cayennensis (sic) (Leptodon cayanensis), in ventriculo; Falco unicinctus (Parabuteo unicinctus), Junio, in oesophago et ventriculo;
Falco 773 (Buteo minumus in Pelzeln, 1871), Buteo brachiurus, Julio, in ventriculo.

Remarks: The exemplars used by Molin (1860d) to describe Physaloptera acuticauda were the same as those used to identify Physaloptera alata by Diesing (1851) of Falco from America.

Status: Physaloptera alata Rudolphi, 1819.

Filaria alcedinis Rudolphi, 1819

Filaria alcedinis, Rudolphi, 1819: 635.

Filaria physalura, Diesing, 1851: 276.

Filaria physalura, Molin, 1858: 412.

Filaria alcedinis, Rudolphi, 1819: 635.

Monopetalonema alcedinis, Freitas \& Lent, 1936: 752.

Monopetalonema alcedinis, Vicente et al., 1996: 95.

Material: Alcedinis n. 82 (Cerile amazona in Pelzeln, 1871) (Chloroceryle amazona), in abdomine.

Remarks: The species was described by Rudolphi (1819) from exemplars collected by Olfers in Alcedo n. II (Megaceryle torquatus) and by Natterer in Alcedinis n. 82. Diesing (1851) described Filaria physalura Bremser, a name not published but in the catalogue of the Museum of Vienna. Molin (1858) considered alcedinis as synonym of physalura, but it was Freitas and Lent (1936) in their review of Monopetalonema who established the priority of the name alcedinis of Rudolphi.

Status: Monopetalonema alcedinis (Rudolphi, 1819) Freitas \& Lent, 1936.

Filaria alcedinis superciliosae Molin, 1858

Filaria alcedinis superciliosae, Molin, 1858b: 426.

Filaria physalura, Stossich, 1898: 76.

Monopetalonema alcedinis, Freitas \& Lent, 1936: 752.

Monopetalonema alcedinis, Vicente et al., 1996: 95.

Material: Alcedo superciliosa (Chloroceryle aenea) 1 , Caiçara, Octobri, in cavo abdom.

Remarks: Species inquirendae (in agreement with Molin, 1858b).

Status: Synonym of Monopetalonema alcedinis.

Heteracis amblymoria Drasche, 1882

Heteracis amblymoria, Drasche, 1882b: 139, Taf. XII, fig. 1-3.

Material: Caprimulgus campestris (Podager nacunda).

Remarks: Drasche does not give a locality or date of collection. In Pelzeln (1871) there are 34 exemplars from 14 localities of the host, but it is not possible to precisely determine the original locality.

Status: Ascaridia amblymoria (Drasche, 1882) Railliet \& Henry, 1914. 
Ophiostomum amphiacanthum Diesing, 1851

Ophiostomum amphiacanthum, Diesing, 1851: 246.

Ophiostomum amphiacanthum, Diesing, 1857: 16, Taf.

II, fig. 29-36.

Rictularia amphiacanthum, Drasche, 1882a: 123.

Rictularioides amphiacanthum, Hall, 1916: 168.

Material: Lemmus dasytrichus (Oxymycterus rufus), 1 q (2 qq, in Drasche, 1882a), Ytararé, 1-ix-1820, in intestinis tenuibus.

Status: In agreement with Quentin (1969). The original description is not enough to identify the species, and probably these are young exemplars of Pterygodermatities, so this should be species inquirenda.

\section{Deletrocephalus amphisbaenae Molin, 1861}

Deletrocephalus amphisbaenae, Molin, 1861a: 577.

Material: Amphisbaena kingii (Anops kingii), 1 है, Caiçara, 5-vi-1826.

Remarks: Molin himself included the species as species inquirenda.

Status: Species inquirenda.

\section{Spiroptera anabatis Molin, 1860}

Spiroptera anabatis, Molin, 1860a: 979.

Spiroptera anabatis, Drasche, 1883b: 206.

Material: Anabates scandens (Automolus leucophthalmus), 2 o 9 , inter tunicas ventriculi, Brasilia.

Remarks: Species inquirenda in agreement with Molin (1860a). Drasche (1883b) belives Spiroptera anabatis is identical with Spiroptera erecta, but $S$. anabatis is twice as long as S. erecta, and in the original descriptions of Molin (1860b) the vulva in S. erecta is in the middle of the body and in S. anabatis is in the posterior part of the body, so the two species are different.

Status: Until the original material is reviewed, it should be considered species inquirenda.

\section{Spiroptera anacanthura Molin, 1860}

Spiroptera anacanthura, Molin, 1860b: 966.

Spiroptera anacanthura, Drasche, 1883b: 203, Taf. XIV,

fig. 15 und 16 .

Oxyspirura anacanthura, Stossich, 1898: 123.

Material: Crotophaga ani, $9 \hat{\jmath}, 2$ 우, Matogrosso, 26ii-1829; Crotophaga major, 4 ふैगे, 6 우, Cuyaba, 24-i-1825, sub eorum membrana nictitante.

Status: Oxyspirura (Barusispirura) anacanthura (Molin, 1860) n. comb.

\section{Filaria annulata Molin, 1858}

Filaria annulata, Molin, 1858b: 386, Taf. I, fig. 5-7. Filaria annulata, Stossich, 1898: 59.
Material: Cebus lagothrix (Lagothrix lagotricha ?), $4 \hat{\jmath} \hat{\jmath}$, 4 우, Brasilia.

Remarks: Molin (1858b) described the species with striated cuticula and 4 big caudal papillae. Two neotropical genera are parasites of monkeys: Dipetalonema (Bain et al., 1986) and Mansonella (Tetrapetalonema) (Bain et al., 2015).

Status: Until the original material is reviewed, the species is incertae sedis.

\section{Spiroptera anolabiata Molin, 1860}

Spiroptera anolabiata, Molin, 1860a: 981.

Spiroptera anolabiata, Drasche, 1883b: 206, Taf. XII, fig. 23.

Filaria anolabiata, Stossich, 1898: 51.

Thelazia anolabiata, Railliet \& Henry, 1910a: 216.

Thelaziella anolabiata, Skrjabin et al., 1967: 66.

Material: Crax fasciolata, 4 q, Rio Cabacalo, 7-viii1825, sub membrana nictitante; same host, 1 , Villa Maria, 16-x-1827, libere ad oculum.

Remarks: Species inquirenda in agreement with Molin (1860a) because he examined 5 females not in good condition.

Status: Thelazia (Thelaziella) anolabiata (Molin, 1860) n. comb.

Physaloptera anomala Molin, 1860

Physaloptera anomala, Molin, 1860d: 650.

Physaloptera anomala, Drasche, 1882a: 128, Taf. VIII, fig. 1-3.

Physaloptera anomala, Ortlepp, 1922: 1021.

Material: Felis onça (Panthera onca), $3 \hat{\jmath}, 1$ +, Matogrosso, in ventriculo.

Status: Physaloptera anomala Molin, 1860.

Spiroptera anterohelicina Molin, 1860

Spiroptera anterohelicina, Molin, 1860a: 975.

Material: Bradypus tridactylus (Bradypus variegatus), 16 오, Borba, 2-vii-1830, in ventriculo.

Remarks: Species inquirenda in agreement with Molin (1860a).

Status: The species has been overlooked, but undoubtedly it is a Spirocercidae; however, until the original material is reviewed the status should be incertae sedis.

Ascaris anterospiralis Molin, 1860

Ascaris anterospiralis, Molin, 1860e: 337.

Ascaris anterospiralis, Diesing, 1861: 660.

Ascaris anterospiralis, Drasche, 1882a: 130.

Material: Felis concolor (Puma concolor), $3 \hat{\delta} \lambda, 5$ 우, Caiçara, 19-xi-1825, in ventriculo.

Remarks: I agree with Drasche (1882); the species is close to Ascaris mystax. 
Status: Close to or the same as Toxocara mystax (Zeder, 1800), so species inquirenda.

Filaria anticlava Molin, 1858

Filaria anticlava, Molin, 1858b: 381.

Dipetalonema anticlava, Lent \& Freitas, 1942: 277.

Dipetalonema anticlava, Vicente et al., 1998: 167.

Material: Dasypus gilvipes (Euphractus sexcinctus), 1 h, Aldea S. Anna, Junio, ad ventriculum.

Status: Dipetalonema anticlava (Molin, 1858) Lent $\&$ Freitas, 1942.

Heteracis anulata Molin, 1860

Heteracis anulata, Molin, 1860e: 340.

Heteracis annulata (sic), Diesing, 1861: 643.

Material: Ophis saurocephalus (Xenodon severus), 1 , , 2 우, Caiçara, 20-vi-1826, in intestino.

Status: The species is still unknown but agrees with the description of a Heterakinae; incertae sedis at this time.

Spiroptera anulata Molin, 1860

Spiroptera anulata, Molin, 1860a: 983.

Spiroptera anulata, Stossich, 1898: 111.

Material: Ciconia maguari, 1 , Forte do Rio Branco, 22-iv-1831, in oesophago.

Remarks: Species inquirenda in agreement with Molin (1860a) and Stossich (1898).

Status: The species has been overlooked, and Molin (1860a) had only a female in good condition, but until the material is reviewed the species should be species inquirenda.

\section{Strongylus anulatus Molin, 1860}

Strongylus anulatus, Molin, 1860e: 350.

Strongylus anulatus, Molin, 1861a: 513.

Strongylus anulatus, Diesing, 1861: 720.

Strongylus anulatus, Stossich, 1899: 128.

Strongylus nigricinctus, Railliet, 1898: 173.

Trichostrongylus nigricinctus, Travassos, 1921: 33.

Material: Palamedea cornuta, $3 \hat{\partial} \hat{\partial}, 10+q$, Egenho do Cap Gama, 23-viii-1826, proventriculus.

Remarks: Species inquirenda in agreement with Stossich (1899). The species, briefly described by Molin, was renamed by Railliet (1898) as Strongylus nigricinctus, not Strongylus annulatus Siebold 1848, and moved to Trichostrongylus by Travassos (1921). Siebold (1848) in a footnote wrote "Strongylus annulatus mihi (aus der Luftröhre des Wolfs)." Nothing else like this species was described or named as new species; as result of this Strongylus annulatus Sielbold, 1848 is a nomen nudum, and the new name, Strongylus nigricinctus, is also nomen nudum.
Status: The species, probably an Ornithostrongylus, is still unknown and incertae sedis.

Spiroptera appendiculata Molin, 1860

Spiroptera appendiculata, Molin, 1860a: 982.

Material: Herypyga helias (Eurypyga helias), 6 + + , Praja de Cujutuba, 12-iii-1835, inter tunicas ventriculi.

Remarks: Species inquirenda in agreement with Molin (1860a).

Status: The species has been overlooked since Molin (1860a); until review of the material, it should be species inquirenda.

Kalicephalus appendiculatus Molin, 1861

Kalicephalus appendiculatus, Molin, 1861a: 544.

Material: Ophis coerulens (?), $1 \hat{\delta}, 3$ 우, Caiçara, 29v-1826; Ophis merremii (Waglerophis merremii), 3 के $\hat{0}, 2$ $ㅇ ㅜ$, same locality, Januario; Ophis saurocephalus (Xenodon severus), $1 \hat{\jmath}, 3$ 우우, Borba, 2-vii-1830; same host, $5 \hat{\partial} \hat{\partial}, 18$ 우, Ypanema, 17-viii-1822; Leptophis ahaetulla, 13 $\widehat{\partial} \widehat{\partial}, 3$ 우오, Caiçara, 11-ii-1826; $1 \hat{\jmath}, 1$ ㅇ same host, locality and date; Spilotes pullatus, 25 ふै่, 24 우 , Cuyaba, 8-iii-1824; same host, 1 ㄱ, 3 우, Jacobina, 1-iv-1828; 1 $\delta, 4$ 우의, same host, Matogrosso, 19-xii-1828; Spilotes sp., 5 해서, 11 우우, Barra do Rio Negro, Novembri; same host, $1 \hat{\delta}, 1$, Rio Hiè, 1-vi-1831; Coluber lichtensteinii (Palusophis bifossatus), 1 万人, 2 우우, Ypanema, 2-x-1821; same host and locality, 3 ổ, 3 웅, 1-xi-1821; Liophis cinerascens, 1 ㅇ, Caiçara, 5-vi1826; Liophis bivittatus, 1 +, same locality, 12-iii-1826; Liophis miliaris (Erythrolamprus miliaris), 1 $\lambda, 4$ 우, Ypanema, 25-i-1829; Liophis regius (?), 1 d, same locality, 17-xii-1821; Cloelia plumbea (Clelia plumbea), $8 \hat{0} \delta, 8$ 우, Cuyaba, 15xi-1824 and 9-xii-1824.

Status: Kalicephalus appendiculatus Molin,1861.

Filaria ardeae Molin, 1858

Filaria ardeae (sublingualis), Molin, 1858b: 428.

Material: Ardea exilis (Ixobrychus exilis), November, Rio Araguay, sub lingua.

Remarks: Species inquirendae in Molin (1858b). I agree with Molin (1858b). The name is in the Museum of Vienna catalogue, but he could not find the exemplars.

Status: It is a nomen nudum.

Spiroptera ardeae Molin, 1860

Spiroptera ardeae, Molin, 1860a: 981.

Spiroptera ardeae, Drasche, 1883b: 206, Taf. XI, fig. 28.

Material: Ardea cocoi, 2 우, Barra do Rio Jaure, 22-x1826, inter bases digitorum pedum.

Remarks: Species inquirenda in agreement with Molin (1860a) because he had two exemplars in poor condition. 
Overlooked by authors, but the figure in Drasche (1883b) seems to be an Acuariinae, although the location of the host does not correspond with it.

Status: Until review the material should be incertae sedis.

Filaria ardearum Rudolphi, 1819

Filaria ardearum, Rudolphi, 1819: 636.

Filaria ardearum, Dujardin, 1845: 56.

Filaria ardearum, Diesing, 1851: 282.

Filaria ardearum, Molin, 1858b: 434.

Material: Ardea leuce (Ardea herodias), Rio do Boraxudo, Decembri, extus ad ventriculum in ductu cartilaginoso propio; Ardea cocoi, Barra do Rio Negro, Novembri, extus ad oesophagum.

Remarks: I agree with Dujardin (1845). The specimens collected by Olfers and studied by Rudolphi (1819) are 54 $\mathrm{mm}$ and $120 \mathrm{~mm}$ long. Molin (1858b) identified some exemplars collected by Natterer with this species, but he believed that these are strongylids instead of filarids. After that, the name disappears from the literature. The size and location of the host make it doubtful that it is a strongylid.

Status: Until study of the original specimens occurs, it should be considered as nomen dubium, but in Hartwich (1966) these are not in Berlin; then very probably it will be a nomen nudum.

Spiroptera armata Molin, 1860

Spiroptera armata, Molin, 1860a: 971.

Material: Dicotyles albirostris (Tayassu pecari), 14 ठิ $\hat{\partial}$, 56 우, Caiçara, 26-iv-1826, in ventriculo.

Status: The species has been overlooked, but the original description by Molin (1860a) looks like a species of Texicospirura; however, until review the material should be incertae sedis.

Sclerostomum armatum Rudolphi, 1802

Strongylus equinus, Müller, 1780: 2.

Strongylus armatus, Rudolphi, 1802: 38.

Sclerostoma equinum, Dujardin, 1845: 257.

Sclerostomum armatum, Diesing, 1851: 303.

Material: Equus mulus, Januario, coeco.

Status: Strongylus equinus Müller, 1780.

Filaria attenuata Rudolphi, 1803

Filaria cornicis, Gmelin, 1790: 3033.

Filaria aquilae, Gmelin, 1790: 3033.

Filaria falconis, Gmelin, 1790: 3040.

Filaria strigis, Gmelin, 1790: 3040.

Filaria attenuata, Rudolphi, 1803: 3.

Filaria attenuata, Rudolphi, 1809: 58-59.
Filaria attenuata, Rudolphi, 1819: 4, 208.

Filaria attenuata, Diesing, 1851: 266.

Filaria attenuata, Molin, 1858b: 394.

Material: Falco peregrinus, Martio, Brasil; Falco swainsonii (Gampsonyx swainsonii), Martio, in cavo orbitae; Falco swainsonii (Gampsonyx swainsonii), Julio, Brasil, inter musculus maxillae inferioris; Strix torquata (Pulsatrix perspicillata) and Strix perlata (Tyto alba), Octobri, Brasil, in abdomine (Diesing). Corvus cyanomelas (Corvus cyanomelas), 6 9 오, in Brasil, in eorum abdomine; Cassicus viridis (Psarocolius viridis), 4 ổ, 3 q $q$, Barra do Rio Negro, Septembri, in cavit. et inter tunicas parietum abdom.; Cassicus yuracares (Psarocolius bifasciatus), 1 j, 1 \&, Matogrosso, Novembri, in pulminibus (Molin).

Remarks: In agreement with Anderson (1959) that samples from corvids are Diplotriaena tricuspis (Fedschenko, 1874) Henry \& O'zoux, 1909, and samples from falcons are Filaria foveolata Molin, 1858 that could be Serratospiculum tendo (Nitzsch, 1857).

Status: Nomen dubium.

Filaria attenuato-verrucosa Molin, 1858

Filaria attenuato-verrucosa, Molin, 1858b: 394.

Filaria attenuato-verrucosa, Stossich, 1898: 60.

Diplotriaena attenuato-verrucosa, Henry \& O'zoux, 1909: 547.

Diplotriaena attenuato-verrucosa, Diaz-Ungría, 1964: 201.

Diplotriaena attenuato-verrucosa, Vicente et al., 1996: 92.

Material: Thamnophilus canadensis (Sakesphorus ca-

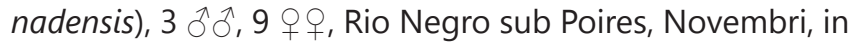
cavo abdom. et thorac.

Remarks: The species was redescribed by Diaz-Ungria (1964).

Status: Diplotriaena attenuatoverrucosa (Molin, 1858) Henry \& O'zoux, 1909.

Strongylus attenuatus Molin, 1860

Strongylus attenuatus, Molin, 1860e: 351.

Strongylus attenuatus, Molin, 1861a: 511.

Strongylus attenuatus, Diesing, 1861: 720.

Strongylus molini, Railliet, 1898: 173.

Strongylus rubidius, Hassal \& Stiles, 1892: 207.

Hyostrongylus rubidus, Hall, 1921: 544.

Material: Dicotyles albirostris (Tayassu pecari), $3 \hat{0} \widehat{0}, 34$ 우, Caiçara, 26-iv-1826, in ventriculo.

Remarks: Railliet (1898) renamed Strongylus attenuatus of Molin (1860) as Strongylus molini because the same name was used by Leidy (1858) for a monkey parasite, but both species actually are in different genera, so the name 
change was unnecessary. The species was transferred to several genera, until it became a synonym of Hyostrongylus rubidius by several authors, but attenuatus takes priority.

Status: Hyostrongylus attenuatus (Molin, 1860) n. comb.

\section{Strongylus auricularis Zeder, 1800}

Strongylus auricularis, Zeder, 1800: 77.

Strongylus subauricularis, Rudolphi, 1819: 649.

Strongylus auricularis, Molin, 1861a: 509.

Oswaldocruzia subauricularis, Travassos, 1917a: 73.

Material: Docidophryne agua (Rhinella icterica), $3 \hat{\partial} \hat{\partial}$, 6 우; same host, 3 ô $\widehat{\partial}, 14$ 웅, Ypanema, 11-xi-1821; same host and locality, 3 ôे $\delta$, 5 우, 4-xii-1819; Cystignathus pachypus (Leptodactylus latrans), 1 ô, 1 \%, Ypanema, 25-x-1821; Ceratophris varia (Ceratophris aurita), 3 + $9, \mathrm{Yp}-$ anema, 21-xii-1821; same host and locality, 1 j, 3 우, 26ix-1821; same host, 2 ô, 5 우, Paranagoa, 1-i-1821; Otolophus cinctus (Rhinella crucifer), $4 \hat{0} \partial^{\lambda}, 10$ q + , Ypanema, 9-ii-1820; same host, 11 के $\hat{\sigma}, 30$ 우, Paranagoa, 1-ii-1821.

Status: Oswaldocruzia subauricularis (Rudolphi, 1819) Travassos, 1917.

\section{Ascaris auriculata Rudolphi, 1819}

Ascaris auriculata, Rudolphi 1819: 655.

Ascaris auriculata, Diesing, 1851: 185.

Ascaris auriculata, Schneider, 1866: 46.

Ascaris auriculata, Hartwich, 1972: 192.

Material: Colubri n. 20 (Rudolphi). Opheomorphus miliaris, in intestinis (Diesing).

Status: Ascaris (s. I.) auriculata (Hartwich, 1972), species inquirenda.

\section{Filaria bacillaris Molin, 1858}

Filaria bacillaris, Molin, 1858b: 379.

Filaria bacillaris, Stossich, 1898: 60.

Oswaldofilaria bacillaris, Travassos, 1933: 160.

Oswaldofilaria bacillaris, Vicente et al., 1993: 93.

Material: Chamsa nigra (Melanosuchus niger), 1 , Matogrosso, Septembri; Champsa sclerops (Caiman crocodilus) 1 , Cuyaba, Majo. In eorum pulmonibus.

Status: Oswaldofilaria bacillaris (Molin, 1858) Travassos, 1933.

\section{Dochmius bidens Molin, 1861}

Dochmius bidens, Molin, 1861a: 493.

Ancylostoma bidens, Freitas, 1951: 191.

Dochmius bidens, Biocca, 1961: 17, Fig. 1 and 2.

Dochmius bidens, Freitas \& Rodrigues, 1964: 48.

Ancylostoma bidens, Vicente et al., 1998: 4.

Material: Procyon cancrivorus, $7 \hat{\partial} \hat{\delta}, 10$ 우, Ypanema, 30-vi-1820; Nasua narica (Nasua nasua), $6 \hat{\jmath} \hat{0}, 11$ 우의 as follows: 2 from Ypanema, 11-ii-1819; 4, same locality, 25$\mathrm{x}-1825 ; 11$ (12?), Las Flechas, 18-vii-1825.

Status: Ancylostoma bidens (Molin, 1861) Freitas, 1951.

Filaria bidentata Molin, 1858

Filaria bidentata, Molin, 1858b: 401.

Setaria bidentata, Railliet \& Henry, 1911a: 387.

Setaria bidentata, Vicente et al., 1998: 175.

Material: Cervus nambi (Mazama nana), $2 \hat{\mathrm{o}} \hat{0}, 2$ 우우, Caiçara, Martio; Villa Maria, Septembri; Cervus simplicicornis (Mazama gouazoubira), 1 \%, 6 우, Matodentro, Decembri; Cervus rufus (Mazama americana), 3 우, Borba, Junio et Decembri. In eorum cavo abdom.

Status: Setaria bidentata (Molin, 1858) Railliet \& Henry, 1911.

Filaria bifida Molin, 1858

Filaria bifida, Molin, 1858b: 411.

Dicheilonema bifidum, Diesing, 1861: 707.

Molinema bifida, Freitas \& Lent, 1939: 15.

Molinema bifida, Vicente et al., 1998: 173.

Material: Dactylomys amblyonyx (Kannabateomys amblyonyx), 1 $\hat{\text {, }} 1$ , Ypanema, Majo, in hepate.

Status: Molinema bifida (Molin, 1858) Freitas \& Lent, 1939

Filaria bifurca Molin, 1858

Filaria bifurca, Molin, 1858b: 400.

Material: Muscicapae sp. Nr. 598 (Hemitriccus margaritaceiventer), $2 \hat{\jmath} \hat{0}, 3$ 우, Cuyaba, Augusto; Muscicapae sp. Nr. 562 (Elaenia cristata), 1 ઈิ, 4 우, Cidade do Gojaz, Septembri. In eorum abdominae.

Status: The species has gone unnoticed in the literature, but by the original description it appears to be a kind of Dicheilonema Diesing,1861, but until the original material is reviewed, it is incertae sedis.

Ancyracanthus bilabiatus Molin, 1860

Ancyracanthus bilabiatus, Molin, 1860e: 343.

Ancyracanthopsis bilabiata, Diesing, 1861: 671.

Ancyracanthus bilabiatus, Drasche, 1883a: 112, Taf. III, fig. 24.

Ancyracanthopsis coronata, Wong \& Lankester, 1985: 1557

Material: Eurypyga helias, $1 \hat{\jmath}, 1$, Praja de Cujatuba, 13-iii-1835, inter tunicas ventriculi.

Remarks: In agreement with Wong \& Lankester (1985). This is a synonym of Ancyracanthopsis coronata (Molin, 1860).

Status: Ancyracanthopsis coronata (Molin, 1860) Chabaud \& Petter, 1959. 
Spiroptera bilabiata Molin, 1860

Spiroptera bilabiata, Molin, 1860a: 928.

Spiroptera bilabiata, Creplin, 1829: 7.

Physaloptera murisbrasiliensis, Diesing, 1861: 653.

Physaloptera murisbrasiliensis, Drasche, 1882a: 128.

Physaloptera murisbrasiliensis, Orplepp, 1922: 1023.

Material: Mus brasiliensis (Holochilus brasiliensis), 3 ते $0^{\lambda}$ 11 우, Ypanema, 19-ii-1819, in ventriculo.

Remarks: Diesing (1861) changed the name to Physaloptera murisbrasiliensis because the Spiroptera bilabiata of Creplin (1829) is a Physaloptera too.

Status: Physaloptera murisbrasilensis Diesing, 1861.

\section{Filaria bipapillosa Molin, 1858}

Filaria bipapillosa, Molin, 1858b: 398.

Material: Strix suinda (Asio flammeus), $8 \hat{\jmath} \delta, 9$ q $\circ$; Ytararé, Januario; same host, Marungaba, Martio; same host, Ypanema, Septembri. Sub cute colli.

Status: As for Filaria bifurca, the species has gone unnoticed in the literature, but by the original description it appears to be a kind of Dicheilonema Diesing, 1861, so incertae sedis.

\section{Filaria bispinosa Diesing, 1851}

Filaria boae constrictoris, Leidy, 1850: 118.

Filaria bispinosa, Diesing, 1851: 278.

Filaria bispinosa, Diesing, 1857: 18, Tav. II, fig. 45-50.

Filaria bispinosa, Molin, 1858b: 415.

Dicheilonema bispinosum (Molin, 1858), Diesing, 1861: 709.

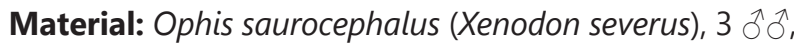
3 우, Junio, in cavo abdominis; same host, Borba, 2-vii1830, inter tunicas intestini; Thamnobius poecilostoma (Pseustes poecilonotus), 1 \&, Rio Içanna, 26-vi-1835, inter membranas oesophagi et pulmonum; Boa constrictor, 2 우의, Mattogrosso, 5-vi-1828, in cavo abdominalis.

Remarks: Walton (1927) did not find Leidy's exemplars, and Filaria boae constrictoris Leidy, 1850 places it in nomen nudum; otherwise, this name has priority for Filaria bispinosa Diesing, 1851.

Status: Must be a Hastospiculum Skrjabin, 1923.

\section{Strongylus bispinosus Molin, 1861}

Strongylus bispinosus, Molin, 1860e: 351.

Strongylus bispinosus, Molin, 1861a: 518.

Strongylus bispinosus, Diesing, 1861: 720.

Haemonchus bispinosus, (Molin, 1860) Railliet \& Henry, 1909a: 86.

Material: Cervus nambi (Mazama nana), 13 ते $\sigma^{2}, 12$ 우, Villa Maria, 16-ix-1825, in ventriculo.

Status: Haemonchus bispinosus (Molin, 1860) Railliet \& Henry, 1909.
Kalicephalus bothropis Molin, 1861

Kalicephalus bothropis, Molin, 1861a: 549.

Alicephalus i. inermis, Schad, 1962: 1091.

Material: Bothrops jararaca, 1 $\delta, 7$, Ypanema, 22-xii-1821.

Remarks: Molin himself included the species as species inquirenda.

Status: Synonym of Kalicephalus i. inermis Molin, 1861.

Ascaris brachyoptera Rudolphi, 1819

Ascaris brachyoptera, Rudolphi, 1819: 275.

Ascaris brachyoptera, Diesing, 1851: 182.

Ascaris brachyoptera, Sprent, 1968: 192.

Material: Nasua narica (Nasua nasua). Brasil.

Remarks: Sprent (1968) included this species as species inquirendae; descriptions are poor and types are lost.

Status: Species inquirendae.

Spiroptera brachystoma Molin, 1860

Ascaris leptocephala, Rudolphi, 1819: 46, 282.

Leiuris leptocephalus, Leuckart, 1850: 11, Taf II, fig. 1

und 2.

Spiroptera brachystoma, Molin, 1860a: 955.

Spiroptera brachystoma, Drasche, 1883b: 201, Taf. XI,

fig. 1-4; Taf. XIII, fig. 10 und 11.

Spiroptera leptocephala, Stossich, 1898: 94.

Acuaria brachystoma, Yamaguti, 1961: 616.

Material: Bradypus tridactylus (Bradypus veriegatus),

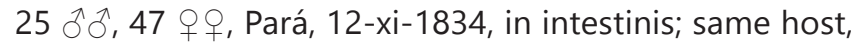

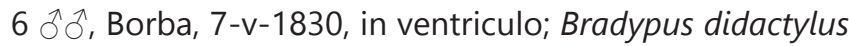
(Choloepus didactylus), 1 , Rio Hiè (Xié), 1-vi-1831, in intestino tenui.

Remarks: Drasche (1883b) suggested that this species is equal to Spiroptera leptocephala, and later Stossich (1898) did it formally.

Status: Leiuris leptocephalus (Rudolphi, 1819) Leuckart, 1850 .

Kalicephalus brevipenis Molin, 1861

Kalicephalus brevipenis, Molin, 1861a: 549.

Kalicephalus brevipenis, Schad, 1962: 1154.

Material: Ophis rhodogaster (?), 2 ô, Cuyaba, 7-vi-1825;

Dryophis fulgidus (Oxybelis fulgidus), $1 \hat{\delta}$, Forte do Pricipe da Beira, 13-viii-1829.

Status: Species inquirenda in Schad (1962).

Spiroptera brevipenis Molin, 1860

Spiroptera brevipenis, Molin, 1860a: 921.

Spiroptera brevipenis, Drasche, 1883b: 194, Taf. XIV, fig.

14.

Oxyspirura brevipenis, Stossich, 1898: 126. 
Material: Dicholophus margravi (Cariama cristata), 17 궁, 21 우오, Cuyaba, 22-iii-1824, sub membrana nictitante.

Remarks: Drasche (1883b) described the species without a buccal capsule, and no newer description exists.

Status: Oxyspirura (Cramispirura) brevipenis (Molin, 1860) n. comb.

Spiroptera brevisubulata Molin, 1860

Spiroptera brevisubulata, Molin, 1860a: 959.

Spiroptera brevisubulata, Drasche, 1883b: 201.

Oxyspirura brevisubulata, Stossich, 1898: 124.

Material: Strix atricapilla (Otus atricapillus), 2 ô $\hat{0}$, 1 , Egenho do Cap Gama, 14-ix-1826, sub membrana nictitante.

Status: Oxyspirura (Barusispirura) brevisubulata (Molin, 1860) n. comb.

Spiroptera bullosa Molin, 1860

Spiroptera bullosa, Molin, 1860a: 937.

Spiroptera bullosa, Drasche, 1883a: 198, Taf. XI, fig. 24;

Taf. XIV, fig. 7 und 8.

? Acuaria bullosa, Yamaguti, 1961: 266.

Cyrnea (Procyrnea) bullosa, Chabaud, 1958: 490.

Material: Tinamus noctivagus (Crypturellus noctivagus), $2 \widehat{\partial} \widehat{\partial}, 2$ 우, Porto do Rio Paraná, 28-v-1823; Tinamus tao, $3 \hat{\partial} \hat{0}, 1$ ㅇ, Barra do Rio Negro, 7-x-1830; Tinamus cinereus (Crypturellus cinereus), 2 ठ̊. Borba, 4-vii-1830; Tinamus variegatus (Crypturellus variegatus), $4 \hat{\partial}$, Borba, 5-vii1830. Inter eorum tunicas ventriculi.

Remarks: The species has been overlooked by several authors. Thanks to the good figures in Drasche (1883a), the species is undoubtedly a Habronematinae and belongs to Procyrnea, as proposed Chabaud (1958).

Status: Species inquirenda Chabaud, 1958.

\section{Filaria calcarata Molin, 1858}

Filaria calcarata, Molin, 1858b: 378, Taf. I, fig. 2.

Filaria calcarata, Diesing, 1861: 701.

Material: Bothrops jararacca, 1 , in cavo abdom.

Status: The species was described from a female without any particular character and has not been subsequently reported, so it is a nomen nudum.

\section{Dispharagus calcaratus Molin, 1860}

Dispharagus calcaratus, Molin, 1860b: 496.

Dispharagus calcaratus, Drasche, 1883b: 210, Taf. XII,

fig. 29 und 30; Taf. XIV, fig. 24.

Acuaria (Hamannia) calcarata, Railliet et al., 1912: 624.

Hamannia calcarata, Stiles \& Hassal, 1920: 540.

Echinuria calcarata, Cram, 1927: 249.

Syncuaria calcarata, Wong et al., 1986: 1189.
Material: Ibis guaruna (Plegadis chihi), 5 q+, Caiçara, 18-iv-1829, inter tunicas ventriculi.

Remarks: The species was studied by Wong et al. (1986), but with the absence of males, the species is placed as species inquirenda.

Status: Syncuaria calcarata (Molin, 1860) Wong et al., 1986 species inquirenda.

Filaria campanulata Molin, 1858

Filaria campanulata, Molin, 1858b: 392.

Thelazia campanulata, Railliet \& Henry, 1910a: 216.

Thelaziella campanulata, Skrjabin et al., 1967: 71.

Thelazia campanulata, Anderson \& Diaz-Ungría, 1959:

73.

Material: Falco magnirostris (Rupornis magnirostris), 1 + , Ypanema, Februario, sub membrana nictitante. 1962)

Status: Species inquirenda (Anderson \& Diaz-Ungria,

Filaria canis brachyuri Molin, 1858

Filaria canis brachyuri, Molin, 1858b: 420.

Material: Canis brachyurus (Chrysocyon brachyurus), 1 exemplar, Registo do Rio Araguay, 20/21-x-1823, sub cuticula in trachea.

Remarks: : Natterer found no complete exemplar and, according to Molin (1858b), there are only fragments, and he gave no description, so it is species inquirendae in agreement with Molin (loc. cit.). See Filaria papillicauda in Pelzeln (1883). Only one Canis brachyurus was collected in Araguay.

Status: The species must be considered nomen nudum.

Dispharagus capitatus Molin, 1860

Dispharagus capitatus, Molin, 1860e: 341.

Dispharagus capitatus, Stossich, 1891: 98.

Acuaria capitata, Railliet et al., 1912: 623.

Dispharynx capitata, Cram,1927: 240.

Material: Falco minutus (Buteo brachyurus), 1 , Matogrosso, 1-vii-1828, in ventriculo.

Remarks: Species inquirendae in agreement with Stossich (1891). In the description of Molin (1860e) the species has cordons markedly flexed, short (perhaps a retraction due to fixation), strongly recurrent, and not anastomosing.

Status: Synhimantus (Dispharynx) capitatus (Molin, 1860) n. comb.

Spiroptera caprimulgi Molin, 1860

Spiroptera caprimulgi, Molin, 1860a: 978.

Material: Caprimulgus megalurus, 1 , Ypanema, 10-x1821, inter tunicas ventriculi.

Remarks: Species inquirenda in agreement with Molin (1860a) because he had only one female in poor condition 
and didn't know whether it was Spiroptera or Dispharagus.

Status: It should be nomen nudum.

Filaria cassici atri Molin, 1858

Filaria cassici atri, Molin, 1858b: 423.

Material: Cassicus ater (Molothrus oryzivorus), 2 우, Caiçara, Martio, in cavo abdominis.

Remarks: Species inquirendae in agreement with Molin (1858b), who examined only two damaged females, but he thought these could be Filaria tridens, a Diplotriaena.

Status: Until the material is reviewed, it should be considered as nomen dubium, although it is possibly a nomen nudum.

\section{Filaria caudispina Molin, 1858}

Filaria gracilis, Rudolphi, 1809: 57 (in partim), less Ce-

bus capucinus and C. apella.

Filaria gracilis, Dujardin, 1845: 46, not Rudolphi, 1809.

Filaria gracilis, Diesing, 1851: 271 (p.p.).

Filaria caudispina, Molin, 1858b: 382, Taf. I, fig. 4.

Dipetalonema caudispina, Diesing, 1861: 703.

Dipetalonema caudispina, Vicente et al., 1998: 168.

Material: Cebus paniscus (Ateles chamek), Octobri; same host, Volta do Campo dos Veados, Julio; Facenda do Padre Battista, Majo; Cebus fatuellus (Sapajus nigritus), Mattodentro, Decembri; same host, Marabitanas, Aprili; same host, Villa Maria, Septembri; same host, Registo do Rio Araguay, Novembri; Cebus arachnoides (Brachyteles arachnoides), Ypanema, Martio; Cebus (Callithrix) sciureus (Saimiri cassiquiarensis) Marabitanas, Aprili; same host, Barra do Rio Negro, Martio, Octubri; Cebus (Callithrix) sciureus (Saimiri sciureus ?), Salto Theotonio, Octobri; Cebus lagothrix (Lagothrix lagotricha), Rio Xie Içanna et Vaupes, Februario; Cebus lagothrix (Lagothrix cana), Salto Theotonio, Octobri; Cebus ursinus (Alouatta caraya), Rio Mamoré, Julio; Ateles variegatus (Ateles paniscus), Serra Arimani; Jacchus rosalia (Saguinus niger), Pará, Novembri et Januario; Cebus ursinus (Alouatta guariba), Ypanema, Septembri. In eorum cavo abdom. Cebus hirsutus (Pithecia mittermeieri), Mamoré, Augusto, sub cute ad plantas manuum; Cebus personatus (Callicebus sp.), inter musculus.

Remarks: Confused by several authors with Dipetalonema gracilis. Stossich (1898) places it as a synonym for Gongylonema filiforme without any explanation. Perhaps more than one species.

Status: Dipetalonema caudispina (Molin, 1858) Diesing, 1861.

Filaria cebi carayae Molin, 1858

Filaria cebi carayae, Molin, 1858b: 418.

Filaria cebi carayae, Stossich, 1898: 132.
Material: Cebus caraya (Allouatta caraya), Villa Maria, Julio, in cavo abdom.

Remarks: The species was never described, and the only reference is in Stossich (1898), who named it a synonym with Gongylonema filiforme Molin, 1857 without reason. Species inquirendae in agreement with Molin (1858b). There is no description in agreement with Molin (loc. cit.); he could not achieve a precise character from this filarid.

Status: It is a nomen nudum.

Filaria cebi trivirgati Molin, 1858

Filaria cebi trivirgati, Molin, 1858b: 418.

Filaria cebi trivirgati, Stossich, 1898: 132.

Material: Cebus trivirgatus (Aotus azarae), Matogrosso, Octobri, cav. abd.

Remarks: Same as that in Filaria cebi carayae.

Status: It is a nomen nudum.

Spiroptera cephaloptera Molin, 1860

Spiroptera cephaloptera, Molin, 1860a: 956.

Cheilospirura cephaloptera, Diesing, 1861: 686.

Cheilospirura cephaloptera, Drasche, 1883b: 212, Taf.

XIV, fig. 17; Taf. XI, fig. 22.

Oxyspirura cephaloptera, Drasche in Stossich, 1898: 124.

Material: Momotus brasiliensis (Momotus momota), 3 ổ, 8 q 9 , Pará, 3-xii-1834; Icterus croconotus (Ostinops decumanus), 2 ôे, Cuyaba, 6-v-1824. Sub eorum membrana nictitante.

Status: Oxyspirura (Barusispirura) cephaloptera (Molin, 1860) n. comb.

Spiroptera cesticillus Molin, 1860

Spiroptera strongylina, Diesing, 1851: 211 (in partim).

Spiroptera cesticillus, Molin, 1860a: 926.

Spiroptera cesticillus, Drasche, 1883b: 195, Taf. XII, fig.

10 und 11.

Texicospirura cesticillus, Chabaud \& Bain, 1981: 78.

Material: Dicotyles albirostris (Tayassu pecari), 1 के, 7 우, Caiçara, 1-ii-1826, in ventriculo.

Status: Texicospirura cesticillus (Molin, 1860) Chabaud \& Bain, 1981.

Strongylus cesticillus Molin, 1861

Strongylus cesticillus, Molin, 1861a: 516.

Trichostrongylus cesticillus, Travassos, 1918a: 196.

Material: Cebus fatuellus (Sapajus apella), 28 $\widehat{\partial}, 32$ 우, Matogrosso, 11-xii-1828.

Status: Trichostrongylus cesticillus (Molin, 1861) Travassos, 1918 
Spiroptera chrisoptera Molin, 1858

Spiroptera chrisoptera, Molin, 1858a: 275, Tav. I, fig. 1-6.

Spiroptera chrisoptera, Molin, 1860a: 945.

Spiroptera chrisoptera, Diesing, 1861: 677.

Syn. of Spiroptera mediospiralis, Molin,1860,

Drasche,1883b: 200.

Syn. of Spiroptera mediospiralis, Stossich, 1898: 95.

Physocephalus nitidulans (Schneider, 1866: 97), Ciurea, 1912: 292.

Syn. of Physocephalus mediospitralis ?, Yorke \& Maplestone, 1926: 309.

Physocephalus chrysoptera (sic), Lopez-Neyra, 1951: 100.

Simondsia chrisoptera, Lopez-Neyra, 1951: 175.

Tejeraira mediospiralis, Diaz-Ungria, 1963: 901.

Material: Tapirus americanus (Tapirus terrestris), Barra do Rio Jagnaricata, Aprili, in excrescentis tuberosis membranae mucosae ventriculi.

Remarks: In Drasche (1883b) it is "identical to Sp. mediospiralis," and it was followed by several authors, but according to the original description and figures, the species is characterized by having four wings, two cephalic and two lateral, spicules very dissimilar, and vulva near the anus the same as Tejeraia.

Status: Tejeraia mediospiralis (Molin, 1858) Diaz-Ungria, 1963.

Spiroptera circularis Molin, 1860

Spiroptera circularis, Molin, 1860a: 922.

22.

Spiroptera circularis, Drasche, 1883b: 194, Taf. XIII, fig.

Spiroptera circularis, Stossich, 1898: 87.

Pelecitus circularis, Railliet \& Henry, 1910b: 251.

Material: Corvus cyanomelas (Cyanocorax cyanomelas), $6 \AA \widehat{o}, 9$ 우, Caiçara, 17-iv-1828 and 21-iv-1828, libere inter tendines ad articuletiones pedum; Corvus cristatellus (Cyanocorax cristattellus), 10 우, Matogrosso, 4-xi-1826, in cystibus ad et inter digitos pedum; Psittacus ararauna (Ara ararauna), 6 ठ人, 6 우, Tagnaral apud, Insua, 23-xi-1823; Psittacus hyacinthinus (Anodorhynchus hyacinthinus), 3 oे $\hat{\text {, }}$, 11 우오, Registo do Rio Araguay, 8-x-1823; same host, Nas Flechas, 17-vii-1825, inter tendines libere ad calcam; Psittacus macao (Ara macao), $8 \hat{\jmath} \hat{\jmath}^{2}, 13$ $q+$, Nas Flechas, 11-vii1825 , inter tendines libere ad calcum; same host, Ponte do Guaporé, 28-ix-1827, ad basim digitorum; Psittacus agilis (Amazona amazonica), 6 우, Cuyaba, 13-ii-1825, in articulations caleis; same host, 2 우, Villa Maria, 18-ix-1825, in articulationes pedis; Psittacus haemorhous (Aratinga acuticaudata), 2 q, Nas Flechas, 20-vii-1825, in articulatione calcis.
Status: Pelecitus circularis (Molin, 1860) Railliet and Henry, 1910.

Filaria circumflexa Molin, 1858

Filaria circumflexa, Molin, 1858b: 377.

Filaria circumflexa, Stossich, 1898: 64.

Material: Trogon aurantius (Trogon surrucura), 1 q, Egenho do Capitão Gama, Augusto; same host, 2 우, Cidade do Matogrosso, Octobri. In cavo abdom.

Status: The Molin (1858b) description must be an Aproctidae, although they have strong cross-sectional striation. Travassos et al. (1939) point to Aprocta sp. in this host, but until review the material is incertae sedis.

Filaria clavato-verrucosa Molin, 1858

Filaria clavato-verrucosa, Molin, 1858b: 380.

Material: Thamnophilus canadensis (Sakesphorus canadensis), 3 우, Marabitanas, Aprili, extus ad intestina.

Remarks: Molin (1858b) characterizes it from the posterior end widened.

Status: The species has been ignored in the literature and is species inquirenda until review of the material.

Filaria claeliae fasciatae Molin, 1858

Filaria claeliae fasciatae, Molin, 1858b: 431.

Filaria cloeliae (sic) fasciatae, Stossich, 1898: 64.

Material: Cloelia fasciata (Clelia clelia ?), 2 exemplars, Matogrosso, Augusto, in cistibus inter tunicas ventriculi.

Remarks: Species inquirenda in agreement with Molin (1858b). Molin points out that Natterer reports two very long filarias, but he is not sure they are filarias.

Status: Species inquirenda.

Ascaris colymborum Rudolphi, 1819

Ascaris spiculigera, Rudolphi, 1809: 168.

Ascaris colymborum, Rudolphi, 1819: 664.

Ascaris spiculigera, Diesing, 1851: 157.

Contracaecum spiculigerum, Railliet \& Henry, 1912: 256.

Material: Colymbi (Podicipedis) n. 196 (Podices dominicus); Tachybaptus dominicus, in intestinis.

Status: Synonym of Contracaecum spiculigerum (Rudolphi, 1819) Railliet \& Henry, 1912.

Filaria conica Molin, 1858

Filaria conicum, Molin, 1858b: 412.

Dicheilonema conicum, Diesing, 1861: 708.

Material: Cavia acuschy (Myoprocta acouchy), 1 , Barra do Rio Negro, Septembri, in cavo abdom.

Remarks: Diesing (1861) included the species in Dichei- 
lonema but agrees with the original description in that it looks like a Molinema.

Status: Incertae sedis.

Spiroptera conocephala Molin, 1860

Spiroptera conocephala, Molin, 1860a: 943.

Spiroptera conocephala, Drasche, 1883b: 199, Taf. XII,

fig. 12 und 13.

Spiroptera conocephala, Gendre, 1922: 92, figs. 1-7.

Torquatella conocephala, Yorke \& Maplestone, 1926:

373.

Cathematella conocephala, Yamaguti, 1961: 282.

Torquatoides conocephala, Inglis, 1965: 222.

Material: Cuculus cayanus (Piaya cayana), Barra de Rio Negro, May, inter tunicas ventriculi.

Status: Torquatoides conocephala (Molin, 1860) Inglis, 1965.

Filaria convoluta Molin, 1858

Filaria convoluta, Molin, 1858b: 390.

Foleyella convoluta, Travassos, 1929a: 968.

Ochoterenella convoluta, Esslinger, 1966: 215.

Ochoterenella convoluta, Vicente et al., 1991: 607.

Material: Cystignathus gigas (Leptodactylus pentadactylus), 6 우, Ypanema, Novembri; Leptodactylus sibilatrix (Leptodactylus fuscus), 1 exemplar, Ypanema, Novembri. In eorum cavo abdom.

Status: Ochoterenella convoluta (Molin, 1858) Esslinger, 1986.

\section{Eucyathostomum copulatum Molin, 1861}

Eucyathostomum copulatum, Molin,1861a: 462.

Material: Dasyprocta aguti, 59 couples, Caiçara, 20-

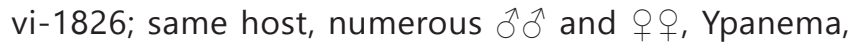
21-x-1821.

Status: Eucyathostomum copulatum Molin,1861.

\section{Spiroptera coronata Molin, 1860}

Spiroptera coronata, Molin, 1860a: 970.

Spiroptera coronata, Drasche, 1883b: 205, Taf. XII, fig. 3 und 4.

Histiocephalus coronatus, Skrjabin, 1916: 511.

Yseria coronata, Gedoelst, 1919: 903.

Skrjabinobronema coronatum, Yamaguti, 1961: 284.

Ancyracanthopsis coronata, Chabaud \& Petter, 1959: 333.

Material: Rallus cayennensis (Aramides cayanea), 1 , 1 piece, Paranagoa, 14-xii-1820; Alcedo americana (Chloroceryle americana), 1 đ̇, 2 우, Praja de Cujutuba, 1835. Inter eorum tunicas ventriculi.
Remarks: Agree with Drasche (1883b) would be Histiocephalus.

Status: Ancyracanthopsis coronata (Molin, 1860) Chabaud \& Petter, 1959.

Histiostrongylus coronatus Molin, 1861

Histiostrongylus coronatus, Molin, 1861a: 532.

Material: Phyllostoma discolor, $4 \hat{\partial} \hat{\delta}, 1+$, Cuyaba, 20-

i-1825; 2 스, 1 ㅇ, same host, Caiçara, 16-iv-1828.

Status: Histiostrongylus coronatus Molin, 1861.

Strongylus costatus Rudolphi, 1819

Strongylus costatus, Rudolphi, 1819: 647.

Sclerostoma costatum, Dujardin, 1845: 260.

Diaphanocephalus costatus, Diesing, 1851: 297.

Diaphanocephalus costatus, Diesing, 1855: 182, Tav. VI, fig. 10-16.

Diaphanocephalus costatus, Molin, 1861a: 587, Tav.

VIII, fig. 6.

Diaphanocephalus costatus, Diesing, 1861: 715.

Kalicephalus costatus costatus, Schad, 1962: 1051.

Kalicephalus costatus, Hartwich, 1971: 306.

Material: Colubri n. 15 (Rudolphi). Lachesis rhombeata (Lachesis muta), Junio; Hylophis laevicollis (Chironius laevicollis), Januario 1818; Herpetodryas bicarinata (Chironius bicarinatus), 11 ก , 3 q, , ex 2 hosts, in intestinis.

Status: Kalicephalus costatus costatus (Rudolphi, 1819) Schad, 1962.

\section{Spiroptera crassicauda Molin, 1860}

Spiroptera crassicanda (sic) (error), Molin, 1860a: 937.

Spiroptera crassicauda, Molin, 1860a: 992.

Spiroptera crassicauda, Drasche, 1883b: 197, Taf. XIII, fig. 16.

Material: Tinamus tao, $10 \hat{\partial} \hat{0}, 7$ q 9 , Barra do Rio Negro, 7-x-1830; Tinamus strigulosus (Crypturellus strigulosus), 10 đô, 5 우, Pará, 19-xii-1834. Inter eorum tunicas ventriculi.

Remarks: There are no other references to this species in the literature, and the Drasche redescription and figure are not sufficient to associate it with any known genus or species.

Status: Until review of the original material, it should be considered incertae sedis.

Dispharagus crassissimus Molin, 1860

Dispharagus crassissimus, Molin, 1860b: 492.

Dispharagus crassissimus, Drasche, 1883b: 209.

Acuaria (Dispharynx) crassisima, Railliet et al., 1912:

623. 
Dispharynx crassissima, Cram, 1927: 243.

Synhimanthus (Dispharynx) crassissima, Pinto et al., 1996: 481.

Material: Rhamphastos vitellinus, 5 q, Serra Arimani apud Rio Branco, 16-vi-1832, inter tunicas ventriculi.

Remarks: Pinto et al. (1996) redescribed the species based on specimens from four species of birds.

Status: Synhimanthus (Dispharynx) crassissima (Molin, 1860) Pinto et al., 1996.

Trichocephalus crenatus Rudolphi, 1809

Trichocephalus suis, Goeze, 1782: 122.

Trichocephalus crenatus, Rudolphi, 1809: 95.

Trichocephalus crenatus, Diesing, 1851: 292.

Trichuris suis, Smith, 1908: 268.

Material: Sus scrofa, Octobri; Dicotyles albirostris (Tayassu pecari), Rio Mamore, Augusto. In corum intestinis crassis.

Status: Trichuris suis (Goeze, 1782) Smith, 1908.

\section{Strongylus crotali Molin, 1861}

Strongylus crotali, Molin, 1861a: 529.

Material: Crotalus horridus (Crotalus durissus), 1 क, Ypanema, 6-xii-1821.

Remarks: Molin himself included the species as species inquirenda.

Status: Species inquirenda.

Trichosoma crypturi Rudolphi, 1819

Trichosoma crypturi, Rudolphi, 1819: 636.

Trichosomum crypturi, Dujardin, 1845: 20.

Trichosomum crypturi, Diesing, 1851: 262.

Capillaria crypturi, Travassos, 1915: 159.

Material: Crypturi (Tinami n. 172); Tinamus tao, intestinis.

Remarks: Species inquirenda in Diesing (1851).

Status: Capillaria crypturi (Rudolphi, 1819) Travassos, 1915.

Oxyuris curvula Rudolphi, 1803

Trichocephalus equi, Goeze, 1782: 117.

Oxyuris curvula, Rudolphi, 1803: 2.

Oxyuris equi, Blanchard, 1849: 170.

Oxyuris curvula, Diesing, 1851: 141.

Material: Equus mulus, Januario, Brasil.

Status: Oxyuris equi (Goeze, 1782) Blanchard, 1849.

\section{Strongylus cylindricephalus Molin, 1861}

Strongylus cylindricephalus, Molin, 1861a: 529.

Trichostrongylus (s.l.) cylindrocephalus, Travassos, 1918a: 191.
Material: Amphisbaena flavescens (Amphisbaena alba), 2 우, Ypanema, 14-iii-1820.

Remarks: Molin himself included the species as species inquirenda.

Status: The species described is supposed to be a Trichostrongylid, but until review of the original material it is a species inquirenda.

\section{Ascaris dactyluris Rudolphi, 1819}

Ascaris dactyluris, Rudolphi, 1819: 272.

Atractis dactylura, Dujardin, 1845: 233.

Ascaris dactyluris, Diesing, 1851: 151.

Atractis d. dactyluris, Petter, 1966: 19.

Material: Rhinemys nasuta (Mesoclemmys nasuta), Februario et Julio; Podocnemis tracaxa (Peltocephalus dumerilianus) Martio; Podocnemis expansa Majo, in eorum intestinis crassis.

Remarks: Petter (1966) restricts the species to Testudo's parasites, and some species are described as Podocnemis from Brazil.

Status: Until the original material is reviewed, it is a species inquirenda.

Filaria dendrocolaptis procurvi Molin, 1858

Filaria dendrocolaptis procurvi, Molin, 1858b: 425.

Material: Dendrocolaptes procurvus (Campylorhamphus trochilirostris), in Brasilia, extus ad oculum.

Remarks: Molin (1858b) had only a single exemplar from which he could not determine the sex, and he placed the species as species inquirendae.

Status: Molin's comment suggests a larva, so this species should be regarded as nomen nudum.

\section{Eucyathostomum dentatum Molin, 1861}

Eucyathostomum dentatum, Molin,1861a: 459.

Material: Dicotyles albirostris (Tayassu pecari), 6 ôे, 45 우, Caiçara, 28-vi-1826; Dicotyles torquatus (Pecari tajacu), 5 ठิ $\hat{0}, 25$ q + , Caiçara, 31-xii-1825. In eorum intestino coeco.

Status: Eucyathostomum dentatum Molin, 1861.

Strongylus dentatus Rudolphi, 1803

Strongylus dentatum, Rudolphi, 1803: 12.

Strongylus dentatum, Diesing 1851: 305 (in partim).

Oesophagostomum dentatum, Railliet \& Henry, 1909b: 169.

Material: Sus scrofa, July and Octobri, in Brazil.

Status: Oesophagostomum dentatus (Rudolphi, 1803) Raillet \& Henry, 1909. 
Stephanurus dentatus Diesing, 1839

Stephanurus dentatus, Diesing, 1839: 232.

Stephanurus dentatus, Diesing, 1851: 296.

Material: Sus scrofa, Barra do Rio Negro, 24-iii-1834,

in lardo, solitarie vel plura capsula inclusa.

Status: Stephanurus dentatus Diesing, 1839.

Spiroptera denudata Rudolphi, 1819

Spiroptera denudata, Rudolphi, 1819: 641.

Spiroptera denudata, Dujardin, 1845: 97.

Spiroptera denudata, Diesing, 1851: 212.

Spiroptera denudata, Molin, 1860a: 920.

Material: Tanagrae n. 124, in intestinis (Rudolphi). Tanagra olivacens (Thraupis palmarum), $3 \hat{0} \hat{0}, 4$ 우, Rio de Janeiro, Decembri, in intestinis.

Remarks: After Molin (1860a), no more references to this species appear in the literature, and the descriptions are not sufficient to associate with any known species.

Status: Until a review of the original material is made, this should be considered species inquirenda.

\section{Ascaris depressa (Zeder, 1800)}

Fusaria depressa, Zeder, 1800: 37.

Ascaris depressa, Rudolphi, 1809: 143.

Ascaris depressa, Rudolphi, 1819: 655.

Ascaris depressa, Diesing, 1851: 156.

Ascaris depressa, Molin, 1861b: 280.

Porrocaecum depressum, Baylis, 1920: 261.

Material: Falco magnirostris (Rupornis magnirostris), Falco brasiliensis (Caracara plancus), Falco degener (Milvago chimachima); Falco rutilans (Heterospizias meridionalis). In intestinis.

Status: Porrocaecum depressum (Zeder, 1800) Baylis, 1920.

\section{Filaria diacantha Molin, 1858}

Filaria diacantha, Molin, 1858b: 381.

Dipetalonema diacanthum, Yorke \& Maplestone, 1926: 427.

Acanthocheilonema diacantha, Boulenger, 1920: 494.

Molinema diacantha, Freitas \& Lent, 1939: 15.

Molinema diacantha, Vicente et al., 1998: 174.

Material: Hystrix (Cercolabes) prehensilis (Coendou prehensilis), 2 $\lesssim$, Villa Maria, 20-viii-1825, ad pulmones in cavo pleurae; same host, 1 , Caiçara, 10-iii-1826, in cavo abdom.; Loncheres rufa (Euryzygomatomys spinosus), $6 \hat{} \widehat{\lambda}$, 12 우, Matogrosso, Octobri, in cav. abdom.

Remarks: In Matogrosso, Natterer collected a specimen of Loncheres armata (Makalata didelphoides) Pelzeln (1883).

Status: Molinema diacantha (Molin, 1858) Freitas \& Lent, 1939.
Physaloptera dilatata Rudolphi, 1819

Physaloptera dilatata, Rudolphi, 1819: 644.

Spiroptera dilatata, Dujardin, 1845: 92.

Physaloptera dilatata, Diesing, 1851: 233.

Physaloptera dilatata, Molin, 1860d: 648.

Physaloptera dilatata, Ortlepp, 1922: 1076.

Material: Simia rosalia (Leontophitecus rosalia) (Rudolphi); Jacchus rosalia (Leontophitecus rosalia), 4 ఓd, 6 우, Sapitiba, 1818; Jacchus vulgaris (Callithrix sp.); Cebus lagothrix (Lagothrix cana), Octobri and Februario; Cebus satanas (Chiropotes satanas), $2 \hat{o} \hat{0}, 4$ 우, Serra Arimani; $\mathrm{Ce}$ bus (Callithrix) caligatus (Plecturocebus caligatus), close to St. Gabriel, Januario; Lagothrix humboldtii (Lagothrix cana), 7 đठ, 4 q 9 , Salto Theotonio, 21-x-1829; Lagothrix hum-

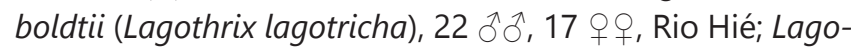
thrix humboldtii (Lagothrix lagotricha), Içanna and Naupe; Cebus apella (Sapajus apella), $3 \hat{\partial} \hat{\sigma}^{2}, 4$ 우, Registo do Rio

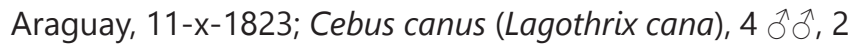
$\rightarrow$, Salto Theotonio, 31-x-1829; Cercopithecus nictitans, 1 ㄱ, 2 우, Rio de Janeiro, 8-iii-1821; Callithrix amictus (Cheracebus torquatus), $1 \hat{\partial}$, St. Barbara, 7 -i-1830. In ventriculo.

Status: Physaloptera dilatata Rudolphi, 1819.

Deletrocephalus dimidiatus Diesing, 1851

Deletrocephalus dimidiatus, Diesing, 1851: 298.

Deletrocephalus dimidiatus, Diesing, 1855: 183.

Deletrocephalus dimidiatus, Molin, 1861a: 575, Tav.

VIII, fig. 1-3.

Deletrocephalus dimidiatus, Diesing, 1861: 714.

Paradeletrocephalus minor, Freitas \& Lent, 1947: 93.

Material: Rhea americana, $186 \hat{\jmath} \widehat{0}, 255$ q $q$, Caiçara,

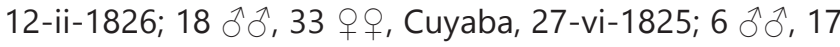
우, Ytararè, 30-viii-1820 and 26-i-1821; Matogrosso, April 1829, in intestino, caeco et recto.

Remarks: Molin described 2 varieties, minor and major; the 2 former samples were minor, the latter is major. Molin's figures referred to minor.

Status: Deletrocephalus dimidiatus Diesing, 1851 and Paradeletrocephalus minor (Molin, 1861) Freitas \& Lent, 1947, respectively.

Filaria dipetala Molin, 1858

Filaria dipetala, Molin, 1858b: 373.

Dipetalonema inflexum, Diesing, 1861: 704.

Filaria dipetala, Stossich, 1898: 67.

Dipetalonema inflexum, López-Neyra, 1956: 150.

Material: Platyrhynchus pitangua (Megarhynchus pitangua), 1 d, Januario, Cuyaba, in cavo abdom.

Remarks: The species has been overlooked in the literature except by Diesing (1861), who renamed it to inflexum without any justification, and this was followed by 
Lopez-Neyra (1956) and then Stossich, who established the synonyms.

Status: Unknown to review materials, species inquirenda.

\section{Sclerostomum dispar Diesing, 1851}

Sclerostomum dispar, Diesing, 1851: 303.

Sclerostomum dispar, Molin, 1861a: 568.

Mammomonogamus dispar, Ryzikov, 1948: 735.

Material: Felis concolor (Puma concolor), $5 \hat{\partial} \hat{\partial}, 5$ 우, Registo do Jauru, 29-vi-1826, in trachea.

Status: Mammomonogamus dispar (Diesing, 1851) Ryzikov, 1948.

Ascaris distans Rudolphi, 1809

Ascaris distans, Rudolphi, 1809: 129.

Ascaris distans, Diesing, 1851: 146.

Primasubulura distans, Inglis, 1958: 599.

Material: Cebus (Callithrix) caligatus (Plecturocebus caligatus), close to St. Gabriel, Januario; Jacchus (Hapale) chrysoleucus (Mico chrysoleuca), Borba, Julio; Jacchus melanurus (Mico melanurus), Matogrosso, Octobri; Jacchus bicolor (Saguinus bicolor), Barra do Rio Negro, Septembri. Reliquae in intestino crasso, rarius coceo.

Status: Primasubulura distans (Rudolphi, 1819) Inglis, 1958.

\section{Ascaris draschei Stossich, 1896}

Ascaris serrata, Drasche, 1883a: 116, Taf. V, fig. 3 und 4.

Ascaris draschei, Stossich, 1896: 24.

Terranova serrata, Mosgovoy, 1951: 20.

Terranova serrata, Moravec \& Justine, 2020: 11.

Material: Vastres cuvieri (Arapaima gigas), 1 1.

Remarks: The species, assumed collected by Natterer and described by Drasche (1883a), was renamed by Stossich (1896) as Ascaris serrata Wright, 1879, but it is not an Ascaris and was described originally as Ancyracanthus serrata (now Cystidicola serratus) and then renamed. The species was not justified.

Status: Species inquirenda, Moravec \& Justine (2020).

\section{Spiroptera erecta Molin, 1860}

Spiroptera erecta, Molin, 1860a: 927.

Cheilospirura erecta, Diesing, 1861: 684.

Cheilospirura erecta, Drasche, 1883b: 211.

Material: Thamnophilus guttatus (Hypoedaleus guttatus), 1 j, 3 웅.

Remarks: Drasche (1883b) makes it synonymous with Spiroptera anabatis, but apparently he had no males. After this author, no more references appear in the literature.

Status: Should be considered incertae sedis until the original material is studied.
Filaria eunectis scytalis Molin, 1858

Filaria eunectis scytalis, Molin, 1858b: 430.

Material: Eunectes scytale (Eunectes murinus), Caiçara, Januario, in pulmonibus.

Remarks: Species inquirendae in agreement with Molin (1858b) and Yamagui (1961).

Status: Molin found only 2 fragments of this species, and there isn't a description, so it must be regarded as nomen nudum.

\section{Filaria eupemphigis marmorati Molin, 1858}

Filaria eupemphigis marmorati, Molin, 1858b: 431.

Foleyella eupemphigis marmorati, Yamaguiti 1961: 116.

Material: Eupemphix marmoratus (Polychrus marmoratus), 2 q + , Caiçara, Octobri, in cavo abdom.

Remarks: Species inquirendae in agreement with Molin (1858b). Foleyella eupemphigis-marmorati (Molin,1858) in Yamaguty (1961), but Molin observed two females in poor condition and studied the genitalia structure and the anterior end. He concludes they are not filarids.

Status: There is no description, so this is a nomen nudum.

Spiroptera excisa Molin, 1860

Spiroptera excisa, Molin, 1860a: 932.

Spiroptera excisa, Drasche, 1883b: 196, Taf. XI, fig. 10

und 11; Taf. XIV, fig. 6.

Cyrnea excisa, Seurat, 1914b: 393.

Excisa excisa, Gendre, 1928: 69.

Hadjelia excisa, Chitwood \& Wehr, 1934: 319.

Material: Ciconia maguari, $52 \lambda \hat{\partial}, 64$ 우, Caiçara, 6-ii1826, in ventriculo et inter ejus tunicas; same host, 21 d $\widehat{d}$, 15 우오, Forte do Rio Branco, 22-iv-1831, in oesophago et proventriculo.

Status: Excisa excisa (Molin, 1860) Gendre, 1928.

Trichocephalus felis Diesing, 1851

Trichocephalus felis, Diesing, 1851: 295.

Trichuris felis, Diaz-Ungría, 1963: 909.

Material: Felis tigrina (Leopardus tigrinus), in intestinis.

Remarks: Species inquirenda in Diesing.

Status: Trichuris felis (Diesing, 1851) Diaz-Ungría, 1963.

Filaria felis mellivorae Molin, 1858

Filaria felis mellivorae, Molin, 1858b: 421.

Filaria felis melivorae, Stossich, 1898: 68.

Material: Felis mellivora (Herpailurus yagouaroundi), Ypanema, Septembri, in pulmonibus.

Remarks: The name Felis mellivora doesn't exist, but under this name in Molin's paper is "Filaria Felis Jaguarondi," 
and Pelzeln (1983) reported Felis jaguarondi in Ypanema in Septembri. Species inquirendae in agreement with Molin (1858b).

Status: Molin (1858b) wrote "of these filariae I found some fragments without anterior end and nor tail," and there isn't a description, so it is nomen nudum.

\section{Filaria felis oncae Molin, 1858}

Filaria felis oncae, Molin, 1858b: 421.

Material: Felis onca (Panthera onca), Septembri, Cachoeira do Robo do Ribeirao, inter musculos.

Remarks: Species inquirendae in agreement with Molin (1858b).

Status: Molin (1858b) found 3 pieces, of which 2 were anterior ends but not tails, so he could not identify the genus. There isn't a description, so it is nomen nudum.

Filaria filiformis Molin, 1858

Filaria filiformis, Molin, 1858b: 396.

Filaria filiformis, Stossich, 1898: 68.

Diplotriaena filiformis, Henry \& O'zoux, 1909: 547.

Diplotriaena filiformis, Anderson, 1959: 260.

Material: Anabates rufifrons (Phacelodomus rufifrons), 1 , Cuyaba, Julio, in cavo abdom.

Status: Diplotriaena filiformis (Molin, 1858) Henry \& O'zoux, 1909. In doubtful status (Anderson, 1959).

\section{Spiroptera filiformis Molin, 1860}

Spiroptera filiformis, Molin, 1860a: 965.

Spiroptera filiformis, Diesing, 1861: 678.

Material: Falco urubutinga (Buteogallus urubutinga), 1 đ’, 8 웅, Egenho do Pará, 21-vi-1825, in muco plicarum ventriculi.

Status: After Molin (1860a), there are no more references to this species in the literature. Until the original material is reviewed, this should be considered incertae sedis.

Ascaris foecunda Rudolphi, 1819

Ascaris foecunda, Rudolphi, 1819: 654.

Ascaris foecunda, Diesing, 1851: 153.

Aplectana foecunda, Yorke \& Maplestone, 1926: 209.

Aplectana foecunda, Travassos: 1931: 263.

Aplectana foecunda, Baker, 1987: 60.

Material: Hyla n. 4. et Rana cornuta (Ceratophris cornuta). In intestinis crassis (Rudolphi). Trachycephalus occipitalis (?), in intestinis crassis (Diesing).

Remarks: Aplectana foecunda in Yorke \& Maplestone, 1926, but Travassos (1931) points out that the description of Rudolphi and Diesing makes it difficult to make a good identification of this species.
Status: Species inquirendae in agreement with Baker (1987).

Heterakis forciparia Schneider, 1866

Ascaris forciparia (sic) forcipata (?), Rudolphi, 1819: 659.

Ascaris strongylina, Rudolphi, 1819: 651.

Heterakis forciparia, Schneider, 1866: 75.

Material: Dicholophus cristatus (Cariama cristata), Caprimulgus urutau (Nyctibius grandis), Caprimulgus nacandua (Podager nacunda), Cuculus seniculus (Cuccyzus minor), Cuculus tingazu (Piaya cayana), Cuculus navius (Tapera naevia), Tetrao uru (?), Bucco sp. Dünndarm. Coecum. Brasilien. Natterer, v. Olfers und Sello.

Status: Schneider (1866) renamed A. forcipata of Rudolphi or more probably a mistake to forcipata, so nomen nudum. See Ascaris forcipata.

Ascaris forcipata Rudolphi, 1819

Ascaris forcipata, Rudolphi, 1819: 659.

Ascaris forcipata, Diesing, 1851: 147.

Subulura forcipata, Railliet \& Henry, 1914: 680.

Material: In coniplurlum avium Brasilienslum, intestinis Natterer reperit, quas singulas recensebo, Cuculus seniculi (Cuccyzus minor); Cuculus naevius (Tapera naevia); Cuculus tingazu (Piaya cayana); Bucco n. 79 (Monasa atra); Caprimulgus urutau (Nyctibius grandis); Caprimulgi n. 94 (Chordeiles acutipennis); Caprimulgus bacaurau (Nyctidromus albicollis) (Rudolphi). Monasa torquata (Ceryle torquata); Monasa tenebrosa (Chelidoptera tenebrosa); Monasa tranquilla (Monasa atra ?), Majo et Septembri; Monasa leucops (Monasa morphoeus), Martio et Julio; Capito collaris (Bucco capensis), Majo; Capito macrorhynchus (Notharchus macrorhynchos), Septembri et Decembri; Capito melanoleucos (Campephilus melanoleucos), Junio et Decembri; Capito tamatia (?), Januario, Aprili, et Augusto; Capito rufiventris (Picumnus rufiventris), Novembri; Capito striolatus (Nystalus striolatus), Julio. In intestinis et coeco (Diesing).

Status: Subulura forcipata (Rudolphi, 1819) Railliet \& Henry, 1914.

Filaria foveolata Molin, 1858

Filaria falconis, Gmelin, 1790: 3040.

Filaria attenuata, Rudolphi, 1819: 4, 208 (p.p.).

Filaria tendo, Nitzsch in Giebel, 1857: 268.

Filaria foveolata, Molin, 1858b: 375.

Serratospiculum foveolata, Skrjabin, 1915: 132.

Serratospiculum tendo Yorke \& Maplestone, 1926: 428.

Material: Falco peregrinus Praja de Cajutuba, Martio, in abdomine; Thamnophilus stagurus (Taraba major) $1 \hat{0}, 1$ 
9. Meia Ponte, Julio, in cavo thorac. et abdom., same host, Goius, Augusto, in cavo abdom.

Remarks: Synonym of Serratospiculum tendo (Nitzsch, 1857) in Yorke and Maplestone (1926) (see Filaria attenuata), but some authors (Yamaguti 1961, Sonin 1968) included under this name some exemplars from Falconiformes, and in other species of Serratospiculum, but Filaria tendo never was described so is a nomen nudum. The status of material from (Taraba) studied by Molin is still doubtful.

Status: Incertae sedis.

\section{Filaria fusiformis Molin, 1858}

Filaria fusiformis, Molin, 1858b: 415.

Dicheilonema fusiforme, Diesing, 1861: 709.

Dicheilonema fusiforme, Vicente et al., 1996: 91.

Material: Monasa tranquilla (Monasa atra), 4 +, Barra do Rio Negro, Octobri, in cavo thorac. sub periostio sterni.

Remarks: The species was described based on females. Diesing (1861) included the species in Dicheilonema, and it is follow by Yamaguti (1961) but is ignored by other authors.

Status: Incertae sedis until posterior study of the exemplars is accomplished.

Diaphanocephalus galeatus (Rudolphi, 1819)

Strongylus galeatus, Rudolphi, 1819: 648.

Sclerostoma galeatum, Dujardin, 1845: 260.

Diaphanocephalus strongyloides, Diesing, 1851: 297.

Diaphanocephalus strongyloides, Diesing, 1855: 182.

Diaphanocephalus strongyloides, Diesing, 1861: 715.

Diaphanocephalus strongyloides, Molin, 1861a: 581.

Diaphanocephalus galeatus, Railliet \& Henry, 1909b: 171.

Material: Lacerta teguixin (Tupinambis teguixin), Novembri (Diesing, 1851). Podinema teguixin (Tupinambis teguixin), Ypanema, i-1818 and 11-xi-1821; same host, 13

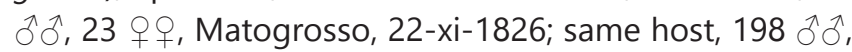
263 우우, as follows: 33 from Cidade de Matogrosso, 29-x1826; 3, Ypanema, 30-ix-1831; same host, many exemplars, Ypanema, 12-xi-1821; same host and locality, 24-xi-1821;

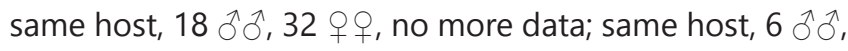
9 우, Ypanema, 26-x-1821; Podinema scripta (Tupinambis teguixin ?) $6 \hat{\jmath} \delta, 11$ 우, Matogrosso, 1-xi-1826; Thorichtis dracaena (Dracaena guianensis), $103 \hat{\jmath}, 113$ q $q$, Matogrosso, 20-v-1828 (Molin). In intestinis.

Status: Diaphanocephalus galeatus (Rudolphi, 1819) Railliet \& Henry, 1909.

Trichocephalus gibbossus Rudolphi, 1819

Trichocephalus gibbossus, Rudolphi, 1819: 639.
Trichocephalus gibbossus, Diesing, 1851: 296.

Oncophora neglecta, Diesing, 1851: 296.

Trichocephalus gibbosa, Hartwich, 1972: 186.

Onchophora gibosa, Baudin, 1970: 988.

Material: ? Thynnus sp., Brasilien; Natterer leg (Hartwich, 1972).

Remarks: Rudolphi (1819) wrote "specimina duo cl. Pohi .. vesicae felleae Scombri Thynni," but Hartwich (1972) points to Natterer as collector. Diesing (1851) renamed the species without comments. The genus was ignored until Baudin Laurencin (1970) revived it, and I agree with him one female of the species is incertae sedis as Onchophora gibossa (Rudolphi, 1819) Baudin, 1970, but in Hartwich (1972) the original material of Natterer is missing.

Status: Nomen nudum.

Eustrongylus gigas Rudolphi, 1802

Ascaris renalis, Goeze, 1782: 39.

Strongylus gigas, Rudolphi, 1802: 115.

Strongylus inflexus, Rudolphi, 1809: 237.

Stenurus inflexus, Dujardin, 1845: 266.

Eustrongylus gigas, Diesing, 1851: 326.

Eustrongylus gigas, Molin, 1861a: 613.

Dioctophyme renale, Stiles, 1901: 167.

Dioctophyma renale, Tollitt, 1987: 238.

Material: Canis jubatus (Chrysocyon brachyurus), Araguay, 20/21-x-1823, in rene dextro; Lutra solitaria (Lontra longicaudis) in rene dextro et in cavo abdominis, Junio.

Status: Dioctophyma renale (Goeze, 1782) Stiles, 1901.

\section{Cheiracanthus gracilis Diesing, 1838}

Cheiracanthus gracilis, Diesing, 1838: 189.

Cheiracanthus gracilis, Diesing, 1839: 225, Taf. XIV, fig.

8-11; Taf. XVII, fig. 1-20.

Cheiracanthus gracilis, Diesing, 1851: 249.

Cheiracanthus gracilis, Drasche, 1882a: 126, Taf. IX, fig. $1-2$.

Gnathostoma gracilis, Stossich, 1901: 1.

Material: Sudis gigas (Arapaima gigas), Borba, 4-i-1830. Im Darmkanal.

Status: Gnathostoma gracilis (Diesing, 1938) Stossich, 1901.

Filaria gracilis Rudolphi, 1809

Filaria gracilis, Rudolphi, 1809: 57.

Filaria gracilis, Diesing, 1851: 271.

Filaria gracilis, Molin, 1858b: 376, Taf. I, fig. 1.

Acanthocheilonema gracilis, Boulenger, 1920: 496, figs 5-7.

Dipetalonema gracilis, Yorke \& Maplestone, 1926: 425.

Dipetalonema gracilis, Vicente et al., 1998: 168. 
Material: Cebus paniscus (Ateles chamek), Julio et Octobri; Cebus apella (Sapajus apella), Mattogrosso, Decembri, in abdomine; Cebus sciureus (Saimiri sp.), Junio, in abdomine; same host, Octobri et Novembri, in musculis intercostalibus; Cebus personatus (Callicebus sp), Decembri, sub cute et inter musculos laterocostales; Cebus hirsutus (Pithecia hirsuta), Borba, Decembri, sub cute palmae manus et inter musculos laterocostales; Cebus variegatus (Ateles paniscus), Cocuy, Martio, in eorum abdomine; Cebus fatuellus (Sapajus nigritus), Registro de Sai, April; Cebus fatuellus (Sapajus apella), Mattogrosso, Septembri; same host, Rio Araguay, Novembri, in cavo abdominis et thoracis; Cebus lagothrix (Lagothrix lagotricha), Julio; Cebus lagothrix (Lagothrix cana), Octobri; Cebus caraya (Alouatta caraya), Villa Maria, Julio; Cebus ursinus (Alouatta sp.), Augusto; Cebus trivirgatus (Aotus sp.), in eorum abdomine; lacchus midas (Saguinus midas), Forte do Rio Branco; lacchus melanurus (Mico melanurus), Caicara, Decembri, inter musculus scapulae et intercostales; lacchus chrysopygus (Leontopithecus chrysopygus); lacchus bicolor (Saguinus bicolor), Barra do Rio Negro, Septembri, in abdomine (Diesing). Cebus apella (Sapajus apella), in autumno, in abdomine et sublingua; same host, Mattogrosso, Novembri, in cavo thorac; Cebus arachnoides (Brachyteles arachnoides), Mattogrosso, Februario, in cavo abdominis; Cebus ursinus (Alouatta guariba), Ypanema, Februario, in cavit. pleurae; Jacchus bicolor (Saguinus bicolor), Barra do Rio Negro, Septembri, in cavo abdominis; Jacchus rosalia (Leontopithecus rosalia?), Mattogrosso, Novembri, in cavo thoracis.

Remarks: Neotropical monkeys are parasitized in different body sites (abdomen, intramuscular, subcutaneous, etc.), so that in this series of samples collected by Natterer and identified as F. gracilis should be several species. A review of the material is necessary.

Status: Dipetalonema gracilis (Rudolphi, 1809) Yorke \& Maplestone, 1926.

Spiroptera gracilis Rudolphi, 1819

Spiroptera gracilis, Rudolphi, 1819: 641.

Spiroptera gracilis, Diesing, 1851: 211.

Spiroptera gracilis, Molin, 1860a: 924.

Physocephalus gracilis, Neiva et al., 1914: 187.

Leiuris gracilis, Vaz \& Pereira, 1929: 3, fig. 9-16.

Ascarops gracilis, Lopez-Neyra, 1951: 116.

Material: Bradypus tridactylus (Bradypus veriegatus), 5 đô, 13 q 9 , Sapitiba, 9-iv-1818, in intestinis.

Status: Physocephalus gracilis (Rudolphi, 1819) Neiva et al., 1914.

Trichocephalus gracilis Rudolphi, 1819

Trichocephalus gracilis, Rudolphi, 1819: 638.
Trichocephalus gracilis, Diesing, 1851: 294.

Trichuris gracilis, Hall, 1916: 23.

Material: Dasyprocta aguti, Octobri; Dasyprocta rufa (Dasyprocta azarae), April, caecum.

Remarks: Species inquirenda in Diesing (1851).

Status: Trichuris gracilis (Rudolphi, 1819) Hall, 1916.

Ascaris granulosa Schneider, 1866

Ascaris spiculigera, Rudolphi, 1809: 168 (p.p.).

Ascaris granulosa, Schneider, 1866: 46, Taf. I, fig. 16.

Contracaecum granulosus, Baylis, 1932: 497.

Contracaecum granulosa, Hartwich, 1964b: 60.

Material: Tachypetes aquilus (Fregata magnificens), oesophagus und ventriculus. Brasilien.

Status: Contracaecum granulosum (Schneider, 1866) Baylis 1932.

Ascaris gulosa Rudolphi, 1819

Ascaris gulosa, Rudolphi, 1819: 271.

Ascaris gulosa, Diesing, 1851: 154.

Labiduris gulosa, Schneider, 1866: 123, Tav. VII, fig.

15-17.

Material: Rhinemys nasuta (Batrachemys nasuta), Junio, in intestinis crassis.

Status: Labiduris gulosa (Rudolphi, 1819) Schneider, 1866.

Spiroptera hamulosa Diesing, 1851

Spiroptera hamulosa, Diesing, 1851: 217.

Spiroptera hamulosa, Molin, 1860a: 959.

Cheilospirura hamulosa, Diesing, 1861: 684.

Cheilospirura hamulosa, Drasche, 1883b: 211, Taf. XII,

fig. 24 und 31; Taf. XIV, fig. 19 und 20.

Dispharagus hamulosus, Stossich, 1891a: 56.

Acuaria (Cheilospirura) hamulosa, Railliet, Henry \& Sisoff, 1912: 623.

Material: Phasianus gallus (Gallus gallus), Septembri, in protuberantia carnosa superficici ventriculi (Diesing). Same host, $5 \partial^{\lambda} \hat{0}, 21$ 우, Cuyaba, 21-ii-1824 (Molin).

Status: Cheilospirura hamulosa (Diesing, 1851) Diesing, 1861.

Filaria helicina Molin, 1858

Filaria helicina, Molin, 1858b: 391.

Paronchocerca helicina, Chabaud \& Ball, 1964: 624.

Paronchocerca helicina, Vicente et al., 1996: 99.

Material: Plotus anhinga (Anhinga anhinga), 2 exemplars plus fragments, Registo do Rio Araguay Octobri; same host and locality, Novembri; same host, Mattogrosso; same host, Marabitanas, Septembri; same host, Caiçara, Aprili et Octobri, inter tunicas cerebri. 
Status: Paronchocerca helicina (Molin, 1858) Chabaud \& Ball, 1964.

\section{Spiroptera helicina Molin, 1860}

Spiroptera helicina, Molin, 1860a: 948.

Spiroptera helicina, Drasche, 1883b: 201, Taf. XIII, fig. 23.

Spiroptera helicina, Stossich, 1898: 87.

Pelecitus helicinus, Railliet \& Henry, 1910b: 251.

Material: Ampelis pompadora (Xipholena punicea),

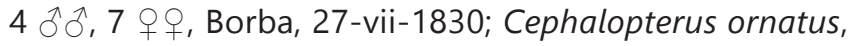

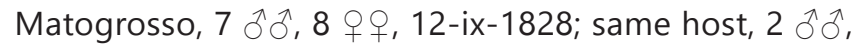
3 우, 12-x-1828; Trogon melanurus, 4 ㅊㅎㅅ, 8 우, Matogrosso, 3-x-1826; Trogon variegatus (Trogon curucui), 2 qq, Villa Maria, 3-ix-1825; same host, 1 d, 3 q $q$, Sao Vicente, 3-vi-1827; Trogon sp., 1 ô, 1 ㅇ, Borba, 12-iii1830; Perdix dentata (Odontophorus capueira), 4 ठえ 우, Pará, Septembri; Perdix rufina (Odontophorus gujanensis), 6 우, Forte do Principe, 13-viii-1829; Urax mitu (Pauxi tuberosa), 4 $\widehat{\delta} \hat{0}, 7$ 우, Matogrosso, 22-viii-1827;

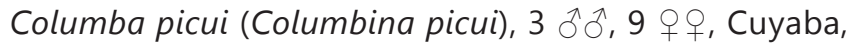
15-i-1824; Cuculus cayanus (Piaya cayana), 1 ô, 1 +, 4-ii1830; Picus comatus (Campephilus melanoleucus), 1 त, 2 우, Cuyaba, 23-iii-1824; same host, 3 우, 7-v-1824; Picus olivinus (Veniliornis passerinus), 4 के $\hat{\partial}, 4$ 우, Cuyaba, 16vi-1824; same host, 3 गेग, 3 우, 24-vii-1824; Psittacus guianensis (Psittacara leucophthalma), 1 ㄱ, 2 우우, Borba, 15-xii-1829; Psittaci hyacinthinus (Anodorhynchus hyacin-

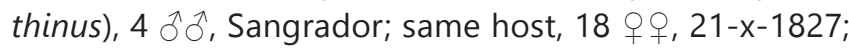
Rhamphastos erythrorhynchus (Ramphastos tucanus), 1 0, 4 우, Pará, 15-xii-1834; Tanagra episcopus (Tangara

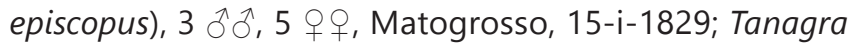
melanops (Trichothraupis melanops), 4 ôे $\hat{0}, 3$ 우, Cuyaba, 21-vi-1824; Cassicus yuracares (Psarocolius bifasciatus), 5 우, Matogrosso, 1-x-1828; Icterus croconotus, 1 औ, Cuyaba, 24-vii-1824; Corvus cyanomelas (Cyanocorax cyanomelas), 2 ôे $\hat{0}, 3$ 우, Cuyaba, 8-v-1825; Thamnophilus sulfuratus (Megarynchus pitangua), $3 \hat{\jmath} \hat{\partial}, 11$ 우우, Cidade de Goyaz, 25-viii-1823; Museicapa despotes (Tyrannus melancholicus), 2 ôे, Cuyaba; same host, 1 + , 19-vi-1825; Museicapa rixosa (Machetornis rixosa), 7 ठ해, 4 우, Cuyaba, 21-vii-1824; Myothera (Formicivora) melanaria (Cercomacra melanaria), 1 ㄱ, 4 우, Cuyaba, 17-ii-1824; Myothera ruficeps (Formicarius colma), $3 \hat{\delta} \hat{0}, 1$, Matogrosso, 2-xi-1826; Turdus fulvus (Molothrus bonariensis), 20 ठै $\widehat{\lambda}, 15$ 우, Tenente Borges, 15-vii-1823; same host, Cuyaba, 3-i1824; Culicivora dumicola (Polioptila dumicola), 5 우, Cuyaba, 6-v-1825; Dendrocolaptes procurvus (Campylorhamphus trochilirostris), $5 \hat{\jmath} \hat{\partial}^{2}$, Cuyaba, 13-vi-1824; Anabates (Annubius) anthoides (Anumbius annumbi), 1 , Borba, 12-iii-1830; Anabates cristatus (Pseudoseisura cristata), $12 \hat{\jmath} \delta^{\lambda}, 5$ 우, Cuyaba, 29-iv-1824; Anabates lusciniodes (Furnarius rufus), $3 \hat{\jmath} \widehat{\partial}, 2$ 우, Cuyaba, 10-vi-1824; same host, 1 ô, 1 +, 27-ix-1824; Alcedo americana (Chloroceryle americana), 1 d , 2 우, Matogrosso, 30-x-1826; Ramphocenus melanurus (Rhamphocenus melanurus), 1 d , 3 우오, Matogrosso, 22-x-1826. Pedis.

Status: Pelecitus helicinus (Molin, 1860) Railliet \& Henry, 1910.

Filaria hemicycla Molin, 1858

Filaria hemicycla, Molin, 1858b: 377.

Filaria hemicycla, Stossich, 1898: 69.

Filaria hemicycla, Vicente et al., 1996: 101.

Material: Psittacus menstruus (Pionus menstruus), Matogrosso, Septembri, prope aurem sub cute colli (neck subcutaneus).

Remarks: Molin (1858b) found only a female and notes that the body curved in a semicircle is characteristic, although the most notable character is the terminal anus.

Status: Incertae sedis in Yamaguti (1961) and Vicente et al. (1996).

Spiroptera heteroclita Molin, 1860

Spiroptera heteroclita, Molin,1860a: 944.

Spiroptera heteroclita, Stossich, 1898: 115.

Oxyspirura heteroclita, Ramson, 1904: 24.

Material: Crax urumutum (Nothocrax urumutum), 1 A, 1 +, Barra do Rio Negro, Aprili 1834, sub membrana nictitante.

Remarks: It's probably an Oxyspirura, but without reviewing the original material it should be considered as incertae sedis.

Status: Oxyspirura (s.l.) heteroclita (Molin, 1860) Ramsom, 1904.

Ascaris heteroptera Diesing, 1851

Ascaris heteroptera, Diesing, 1851: 175.

Ascaris heteroptera, Diesing, 1857: 12, Taf. I, fig 7-13.

Ascaris heteroptera, Drasche, 1882a: 131, Taf. IX, fig. 13-16.

Porrocaecum heteroptera, Hartwich, 1957: 216.

Material: Ibis albicollis (Theristicus caudatus), 2 exemplars, Murungaba, 2-iv-1821; same host, Ytararé, 17-viii1821; 5 exemplars, same host, Mattogrosso, 23-ii-1829; Ibis nudifrons (Phimosus infuscatus), 1 exemplar, Guarauna, 16v-1823; in intestinis tenuibus.

Status: Porrocaecum heteroptera (Diesing, 1851) Hartwich, 1957. 
Filaria horrida Diesing, 1851

Filaria rheae, Owen, 1843: 74.

Filaria horrida, Diesing 1851: 278.

Dicheilonema horrida, Diesing, 1857: 19, Tav. III, fig.

1-5; Tav. IV, fig. 1-25.

Filaria horrida, Molin, 1858b: 416.

Dicheilonema horridum, Diesing, 1861: 709.

Filaria horrida, Stossich, 1898: 48.

Contortospiculum horridum, Skrjabin, 1917: 460.

Contortospiculum rheae, Baylis \& Daubney, 1926: 430.

Dicheilonema rheae, Lent \& Freitas, 1948: 61.

Dicheilonema rheae, Vicente et al., 1996: 91.

Material: Rhea americana, 23 exemplars, Cuyaba, 26iii-1824, in cavo thoracis et abdominis; same host, 2 exemplars, Nas Lages, 4-iv-1823, in cavo thoracis; same host, 1 exemplar, Arica, 21-xii-1823, in cavo thoracis et sub cute coxae; same host, 4 exemplars, Caiçara, 11-ii-1926, in cavo thoracis in duabus cistibus aereis.

Remarks: Several authors, including Sonin (1968) and Brazilians (Vicente et al., 1996), considered Baylis and Daubney (1926) as the authors of this combination; however, these authors do not use it. Lent and Freitas (1948) were the first to use this combination.

Status: Dicheilonema rheae (Owen, 1847) Lent \& Freitas, 1948.

Ascaris hystrix Diesing, 1851

Ascaris hystrix, Diesing, 1851: 188.

Ascaris hystrix, Diesing, 1857: 13, Taf. I, fig. 24-30.

Ascaris hystrix, Drasche, 1882a: 133, Taf. X, fig. 1-4.

Paratractis hystrix, Sarmiento, 1959: 65, fig. 1-8.

Material: Podocnemis erythrocephala, some exemplars, Marabitanas, 21-iv-1831, in intestino recto.

Status: Paratractis hystrix (Diesing, 1851) Sarmiento, 1959.

Filaria hystrix Molin, 1858

Filaria hystrix, Molin, 1858b: 408.

Material: Strix flammea (Asio flammeus), $2 \hat{\partial} \widehat{\partial}, 4$ 우오, Ypanema, Octobri, in cavo abdominis.

Remarks: The species has been overlooked by almost all authors; less by Yamaguti (1961), who places it in incertae sedis. Molin (1858b) characterized the species as having four teeth (?) converged at the anterior end and a body covered with small bosses.

Status: Without reviewing the material, it is incertae sedis.

Spiroptera ibis Molin, 1860

Spiroptera ibis, Molin,1860a: 984.

Material: Ibis rubra (Endocimus ruber), 1 , Praja de
Cujatuba, 22-iii-1835, inter tunicas ventriculi.

Remarks: Species inquirenda in agreement with Molin, who examined only one female.

Status: Probably it is a nomen nudum.

Filaria icteri pyrrhopteri Molin, 1858

Filaria icteri pyrrhopteri, Molin, 1858b: 423.

Material: Icterus pyrrhopterus (Icterus cayenensis), 1 exemplar, Porto do Rio Paraná, April, in cavo abdominis regione renali.

Remarks: Species inquirendae in agreement with Molin (1858b). Molin did not think this is a filarial worm with a bilabiate mouth; also, he had only a very dark specimen for examining the genitalia.

Status: Should be nomen nudum.

Spiroptera imbricata Molin, 1860

Spiroptera imbricata, Molin, 1860a: 972.

Spiroptera imbricata, Drasche, 1883b: 205, Taf. XI, fig.

27; Taf. XIII, fig. 15.

Material: Tantalus loculatos (Mycteria americana), $2 \widehat{\partial} \widehat{\partial}$, 2 우, Caiçara, 8-iii-1821, inter tunicas ventriculi; same host, 2 exemplars, Matogrosso, 7-iv-1828.

Remarks: After Drasche (1883b) no further references are made in the literature to this species. Agrees with his figures and description, it would be a species of Acuariidae.

Status: Until a review of the original material is accomplished, it should be considered incertae sedis.

Filaria incrassata Molin, 1858

Filaria incrassata, Molin, 1858b: 389.

Dirofilaria incrassata, Sandground, 1938: 422.

Dirofilaria incrassata, Vicente et al., 1998: 170.

Material: Nasua narica (Nasua nasua), $4 \hat{\partial} \hat{\partial}, 4$ 우, Nas Trechas (sic) (must be Rio das Flechas, Matogrosso), Julio, in paniculo adiposo sub cute abdominis; Bradypus tridactylus (Bradypus variegatus), 5 우, Borba, Martio, in cavo axillari et in tunica superf. ventric. Majoris; same host, Barra do Rio Negro, Aprili, inter fibras diaphragmatis.

Status: Dirofilaria incrassata (Molin, 1858) Sandground, 1938.

Kalicephalus inermis Molin, 1861

Kalicephalus inermis, Molin, 1861a: 541.

Material: Bothrops jararaca, $3 \hat{\jmath} \delta, 5$ 우, Sao Paulo, 7-i1819, esternamente sui polmoni; Crotalus horridus (Crotalus

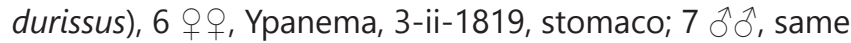
host, same locality, 6-xii-1821; same host, 1 0,1 , same locality, 30-iii-1819, polmoni.

Status: Kalicephalus inermis inermis Molin,1861. 
Physaloptera inflata Molin, 1860

Spiroptera inflata, Molin,1860a: 976.

Spiroptera inflata, Drasche,1883b: 205.

Physaloptera inflata, Stossich, 1889: 51.

Physaloptera inflata, Ortlepp, 1922: 1096.

Physaloptera inflata, Skrjabin \& Sobolev, 1964: 231.

Material: Falco unicinctus (Parabuteo unicinctus), 3 우, Matogrosso, 29-vi-1827, in oesophago et ventriculo.

Remarks: Species inquirenda in agreement with Molin (1860a). Drasche (1883b) wrote "Diese Species ist eine echte Physaloptera" (the species is a true Physaloptera) and differs from Physaloptera acuticauda, also in the falconids. Molin (1860a) got 3 females, and the size is $7.0 \mathrm{~mm}$. Perhaps they are larvae or immature; if that is the case, the species would be nomen nudum. Ortlepp (1922) considered, in his review of Physaloptera, two different species, but he was unable to review the original material, and Morgan $(1943,1946)$ ignores both. In Skrjabin and Sobolev (1964), it is a species inquirenda.

Status: Physaloptera inflata (Molin, 1860) Drasche, 1883 , or nomen nudum if the original material disappears.

\section{Strongylus inflatus Molin, 1861}

Strongylus inflatus, Molin, 1861a: 520.

Bradypostrongylus inflatus, Travassos, 1937: 257.

Material: Myrmecophaga jubata (Myrmecophaga tridactyla), many $\widehat{\delta} \delta$ and $+q$, Cuyaba, 9-ii-1824.

Status: Bradypostrongylus inflatus (Molin, 1861) Travassos, 1937.

Ascaris inflexa Rudolphi, 1809

Fusaria reflexa, Zeder, 1800: 33.

Ascaris vesicularis, Rudolphi, 1809: 129.

Ascaris inflexa, Rudolphi, 1809: 132.

Ascaris inflexa, Diesing, 1851: 169.

Ascaris inflexa, Molin 1861b: 286.

Ascaridia galli, Freeborn, 1923: 223.

Material: Phasiaunus gallus (Odontophorus gujanensis) Cuyaba, in intestini.

Status: Synonym of Ascaridia galli (Schrank, 1788) Freeborn, 1923.

Dipetalonema inflexum Diesing, 1861

Filaria dipetala, Molin, 1858b: 373.

Dipetalonema inflexum, Diesing, 1861: 704.

Material: Platyrhynchus pitangua (Megarynchus pitangua), $1 \hat{\jmath}$, Cuyaba, Januario, in cavo abdominis.

Status: Nomen nudum. See Filaria dipetala.

Peritrachelius insignis Diesing, 1851

Peritrachelius insignis, Diesing, 1851: 210.
Peritrachelius insignis, Diesing, 1855: 181, Taf. V, fig. 8-13.

Peritrachelius insignis, Drasche, 1882a: 128.

Anisakis insignis, Baylis, 1920: 260.

Material: Delphinus amazonicus (Inia geoffrensis), Borba, Januario; same host, Rio Guaporé abaixo da Laterna, 27-vii-1929, in ventriculo.

Status: Anisakis insignis (Diesing, 1851) Baylis, 1920.

Filaria intercostalis Molin, 1858

Filaria intercostalis, Molin, 1858b: 418.

Material: Cebus (Callitrix) sciureus (Saimiri ustus), 4 exemplars, Rio Mamoré, Augusto, inter musculus intercostales.

Remarks: Molin (1858b) examined the specimens but could not identify them with certainty. Species inquirenda in agreement with Molin (1858b).

Status: Until review the material must remain as species inquirenda.

\section{Sclerostomum isotricis Molin, 1861}

Sclerostomum isotricis, Molin,1861a: 560.

Material: Isothrix pachyura (Isothrix b. bistriata), $2 \hat{\partial} \sigma_{1}$ Barra do Rio Negro, 26-iv-1834.

Remarks: Molin himself included the species as species inquirenda.

Status: Until review the material must remain as species inquirenda.

Filaria jacchi chrysopygi Molin, 1858

Filaria jacchi chrysopygi, Molin, 1858b: 419.

Material: Jacchus (Hapale) chrysopygus (Leontopithecus chrysopygus), 1 exemplar, Ypanema, Martio, ad hep.

Remarks: According to Molin, he had a single exemplar that he could not identify. Species inquirenda in agreement with Molin (1858b).

Status: Species inquirenda.

Filaria labiotruncata Molin, 1858

Filaria labiotruncata, Molin, 1858b: 412.

Dicheilonema labiotruncatum, Diesing, 1861: 708.

Tetracheilonema quadrilabiatum, Yorke \& Maplestone, 1926: 439.

Material: Tinamus adspersus (Crypturellus undulatus), 6 우, Borba, May, inter cutem et musculus femoris; Tinamus variegatus (Crypturellus variegatus), $4+q+$, Borba, July, in cavo abdominis; Tinamus strigulosus (Crypturellus strigulosus), 3 웅, Pará, December, inter cutem et musculus pectoris et dorsi.

Status: Synonym of Tetracheilonema quadrilabiatum (Molin, 1858) Diesing, 1861. 
Histiocephalus laciniatus Molin, 1860

Histiocephalus laciniatus, Molin, 1860c: 513.

Histiocephalus laciniatus, Drasche, 1883b: 207, Taf. XII,

fig. 32.

Schistorophus laciniatus, Railliet, 1916: 102.

Material: Rallus cayennensis (Aramides cajanea), 2 え $\lambda$, 3 우, Paranagoa, 14-xii-1820, inter tunicas ventriculi.

Status: Schistorophus laciniatus (Molin, 1860) Railliet, 1916.

Ascaris lanceolata Molin, 1860

Ascaris lanceolata, Molin, 1860e: 334.

Ascaris lanceolata, Drasche, 1882a: 133, Taf. X, fig. 5-11.

Terranova lanceolata, Sprent, 1979b: 266.

Neoterranova lanceolata, Moravec \& Justine, 2020: 9.

Material: Champsa nigra (Melanosuchus niger), more than 400 exemplars, Borba, 27-vi-1830, in ventriculo.

Status: Neoterranova lanceolata (Molin, 1860) Moravec \& Justine, 2020.

Spiroptera lanceolata Molin, 1860

Spiroptera lanceolata, Molin, 1860a: 940.

Spiroptera lanceolata, Drasche, 1883b: 198, Taf. XIV,

fig. 9.

Material: Crotophaga major, $5 \hat{\jmath}, 9$ 우, Cuyaba, Junio, inter tunica ventriculi.

Status: In agreement with Drasche (1883b) that this is a synonym of Cyrnea semilunaris (Molin, 1860).

Ascaris laticauda Molin, 1860

Ascaris laticauda, Molin, 1860e: 335.

Ascaris laticauda, Diesing,1861: 665.

Ascaris laticauda, Drasche,1882a: 132, Taf. IX, fig

17-20.

Heterakis laticaudata, Stossich, 1888: 282, Tav. IV, fig 8; Tav. IX, fig. 42.

Material: Dicholophus marcgravi (Cariama cristata), more than 100 exemplars, Zamambaya, 1-xii-1823, in intestino tenui et coeco.

Remarks: In agreement with Drasche (1882a) "would be Heteracis [sic] better than Ascaris."

Status: Heterakis laticauda (Molin, 1860) Stossich, 1888.

Ascaris leptoptera Rudolphi, 1809

Ascaris leptoptera, Rudolphi, 1809: 137.

Ascaris crenulata, Bremser, 1824: 5, Tab. IV, fig. 24.

Ascaris leptoptera, Diesing, 1851: 179.

Toxocara leptoptera, Hartwich, 1964: 61.

Material: Felis concolor (Puma concolor), Caiçara, Junio 1826; same host, Forte do Rio Branco, May, intestine;
Felis onça (Panthera onca), Caiçara, 26-xii-25; Felis pardalis (Leopardus pardalis), Caiçara, May, intestino crasso; Felis macroura (Leopardus wiedii), intestine.

Remarks: The species has been moved to several genera or is synonymous with several species. Sprent (1959) suspects that Diesing's material is different from Rudolphi's original. Hartwich (1964) reviewed a syntype of lion and identified the species as Toxocara leptoptera.

Status: Until review of the original material collected by Naterer, the species is incertae sedis.

Spiroptera leptoptera Rudolphi, 1819

Ascaris anceps, Fröhlich, 1802: 36.

Spiroptera leptoptera, Rudolphi, 1819: 26 and 247.

Spiroptera intermedia, Creplin, 1825: 11.

Spiroptera leptoptera, Diesing, 1851: 217.

Spiroptera leptoptera, Molin, 1860a: 953.

Filaria leptoptera, Schneider, 1866: 97.

Habronema leptoptera, Seurat, 1914a: 22.

Cyrnea (Procyrnea) leptoptera, Chabaud, 1958: 489.

Material: Falco albicollis (Leucopternis albicolis), Falco aurantius (Falco deiroleucus), November; Falco cachinans (Herpetotheres cachinnans), Caiçara, Octobri; Falco magnirostris (Rupornis magnirostris), Martio; Falco xanthothorax (Microastur ruficollis), Falco bidentatus (Harpagus bidentatus), May; Falco unicinctus (Parabuteo unicinctus), Junio, in eorum ventriculo (Diesing). Falco aurantius (Falco deiroleuCus), $2 \hat{\jmath} \hat{0}, 12$ 우, Caiçara, 31-xii-1825, in eorum ventriculo; Falco cachinans (Herpetotheres cachinnans), 57 के $\hat{0}, 62$ 우의 Caiçara, 24-x-1825; Falco magnirostris (Rupornis magniros-

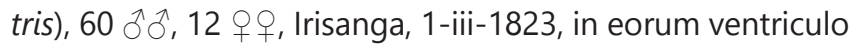
et intestino tenui; Falco xanthothorax (Microastur ruficollis),

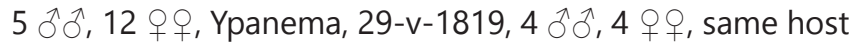
and locality, 24-vi-1819; $5 \hat{\jmath} \hat{0}, 1$ + , Barra do Rio Negro, 4-x1830, in ventriculo; Falco uncinatus (Parabuteo unicinctus), 1 d, 1 , Matogrosso, 29-vi-1827, in oesophago et ventriculo; Falco bidentatus (Harpagus bidentatus), 17 ôd 37 , 37 우, Borba, 8-v-1830, in ventriculo et intestino tenui; Falco tri-

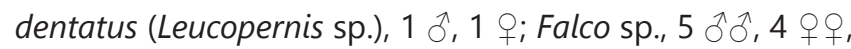
no more data (Molin).

Status: Procyrnea leptoptera (Rudolphi, 1819) Chabaud, 1958.

Dispharagus longeornatus Molin, 1860

Dispharagus longeornatus, Molin 1860b: 489.

Dispharagus longeornatus, Drasche, 1883b: 209.

Dispharagus longeornatus, Stossich, 1891: 96.

Acuaria longeornata, Railliet, Henry \& Sisoff, 1912: 624.

Hammania longeornata, Stiles \& Hassall, 1920: 540.

Echinuria longeornata, Cram, 1927: 255.

Syncuaria longeornatus, Wong et al., 1986: 1194. 
Material: Ciconia maguari, 8 우, Caiçara, 6-ii-1826, in ventriculo et proventriculo.

Remarks: Molin (1860b) had only females with cordons long and straight, briefly recurrent, and anastomose in pairs; without mention of spines on the body, then it is a Syncuaria.

Status: Syncuaria longeornatus (Molin, 1960) Wong et al., 1986.

\section{Spiroptera longestriata Molin, 1860}

Spiroptera longestriata, Molin,1860a: 958.

Cheilospirura longestriata, Diesing, 1861: 684.

Cheilospirura longestriata, Drasche,1883b: 212, Taf. XI,

fig. 30 und 31; Taf. XIII, fig. 13; Taf. XIV, fig. 26.

Habronema mansoni (p.p.), Seurat, 1914c: 427.

Habronema longistriatum (Molin, 1859) (sic), Gendre, 1923: 124.

Cyrnea (Procyrnea) longistriata (Molin,1859) (sic), Chabaud, 1958: 490.

Material: Picus campestris (Colaptes campestris), 2 ôे, 6 우, Ypanema, 25-i-22, membrane dei ventricoli; Picus grammicus (Celeus grammicus), 1 ㅅ, 5 우, Marabitanas, 6-v-1830; Picus jumana (Celeus elegans), 5 ㅊㄲ, 5 우오, Borba, 25-vii-1830, inter eorum tunicas ventriculi.

Remarks: Drasche (1883b) notes that the material from Picus grammicus is different with regard to the distribution and number of caudal papillae. The asymmetric caudal alae that led Seurat (1914c) to assign this material to his new species Habronema mansoni was repeated later by other authors. However, the length of the spicules, distribution of caudal papillae, asymmetry of the caudal alae (right is shorter in mansoni and left is shorter on the material of $P$. grammicus), and hosts and geographical distribution (European birds of prey to mansoni and South American woodpeckers to longestriata) makes the material collected by Natterer Picus grammicus, not Habronema (Procyrnea) mansoni, but probably a yet undescribed species.

Status: Procyrnea longestriata (Molin, 1860) Chabaud, 1958.

\section{Spiroptera longesubulata Molin, 1860}

Spiroptera longesubulata, Molin, 1860a: 960.

Spiroptera longesubulata, Diesing, 1861: 667.

Material: Ciconia maguari, $1 \hat{\jmath}, 2$ 우, Forte do Rio Branco, 22-iv-1831, in proventriculs.

Remarks: After Molin (1860a) and other old authors, no further references to this species are in the literature, and the descriptions are not sufficient to associate with any known species.

Status: Until the original material is reviewed, it should be considered species inquirenda.
Eucyathostomum longesubulatum Molin,1861

Eucyathostomum longesubulatum, Molin,1861a: 460.

Material: Cervus campestris (Odocoileus virginianus), $14 \widehat{\partial} \widehat{\partial}, 30$ 우, Forte do Rio Branco, not dated; Cervus rufus (Mazama rufa), $1 \hat{\delta}$, Cuyaba, 28-ii-1824, in intestino coeco.

Status: Eucyathostomum longesubulatum Molin, 1861.

Dispharagus longevaginatus Molin, 1860

Dispharagus longevaginatus, Molin, 1860b: 486.

Dispharagus longevaginatus, Drasche, 1883b: 209, Taf.

XII, fig. 28; Taf. XIV, fig. 25.

Acuaria longevaginata, Railliet et al., 1912: 624.

Desportesius longevaginatus, Wong et al., 1986: 1194.

Material: Ciconia maguari, $8 \hat{\jmath} \hat{\partial}, 26+q$, Forte do Rio Branco, 22-iv-1831, in oesophago.

Status: Desportesius longevaginatus (Molin, 1860) Wong et al., 1986.

Oesophagostomum longipene Molin, 1861

Oesophagostomum longipene, Molin 1861a: 446.

Kiluluma longipene, Travassos, 1929b: 137.

Material: Tapirus terrestris, 1 \&, Ytararè, 10-viii-1820; same host, 1, 1 \& , 2 ?, Barra do Rio Jaguaricata, 8-iii1825; same host, 1 Jे, Matogrosso, iv-1829; same host, 1 ․ Cachocira do Bananeira, 1-ix-1929; 34 exemplars, same host and locality, 4-ix-1829; same host, 1 , Forte do Rio Branco, 1835.

Status: Kiluluma longipene (Molin, 1861) Travassos, 1929.

Ascaris lumbricoides Linnaeus, 1758

Ascaris lumbricoides, Linnaeus, 1758: 648.

Ascaris suum, Goeze, 1782: 71.

Ascaris lumbricoides, Diesing, 1851: 166.

Material: Dicotyles torquatus (Pecari tajacu), no more data.

Remarks: Material from this host belongs to Ascaris suum Goeze, 1782 (Sprent, 1968; Vicente et al., 1998).

Status: Ascaris suum Goeze, 1782.

Ascaris macroptera Diesing, 1851

Ascaris macroptera, Diesing, 1851: 182.

Ascaris macroptera, Diesing, 1857: 13, Taf. I, fig. 18-23.

Ascaris macroptera, Drasche, 1882a: 132, Taf. X, fig. 16-18.

Material: Champsa nigra (Melanosuchus niger), Rabo do Bananeira, Januario 1829.

Remarks: The species has a well-developed cephalic alae like that of Toxascaris, but after Drasche (1882a), the species disappears from the literature. 
Status: Without a review, the specimens are considered to be incertae sedis.

\section{Ascaris maculosa Rudolphi, 1802}

Ascaris maculosa, Rudolphi, 1802: 22.

Ascaris maculosa, Diesing, 1851: 182.

Heterakis maculosa, Schneider, 1866: 72, Taf. III, fig. 11, vergr. 62.

Ascaridia maculosa, Railliet \& Henry, 1914: 678.

Ascaridia maculosa, Hartwich, 1965: 123.

Material: Columba talpacoti (Columbina talpacoti), Septembri; Columba picui (Columbina picui), Cuyaba, Martio; Columba speciosa (Patagioenas speciosa), Engenho do Capitão Gama, July, Guaporé Augusto.

Status: Ascaridia maculosa (Rudolphi, 1802) Railliet \& Henry, 1914.

\section{Dispharagus magnilabiatus Molin, 1860}

Dispharagus magnilabiatus, Molin, 1860b: 497.

Dispharagus magnilabiatus, Drasche, 1883b: 210, Taf.

XIII, fig. 5.

Acuaria (Cheilospirura) magnilabiata, Railliet et al., 1912: 623.

Cheilospirura magnilabiata, Stiles \& Hassall, 1920: 312. Dispharynx magnilabiata, Gendre, 1921: 38.

Material: Platalea ajaja, $4 \hat{\jmath} \widehat{\partial}, 5$ + , Villa Maria, 7-viii1825 , inter tunicas ventriculi.

Status: Synhimantus (Dispharynx) magnilabiatus (Molin, 1860) n. comb.

\section{Physaloptera magnipapilla Molin, 1860}

Physaloptera magnipapilla, Molin, 1860d: 657.

Physaloptera magnipapilla, Drasche, 1882a: 128, Taf.

VIII, fig. 6 und 7.

Physaloptera magnipapilla, Ortlepp, 1922: 1058.

Material: Myrmecophaga bivittata (Tamandua tetra-

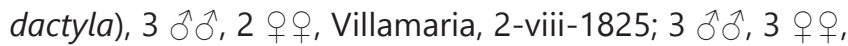
Caiçara, 11-xi-1825, in ventriculo.

Status: Physaloptera magnipapilla Molin, 1860.

\section{Dispharagus mamillaris Molin, 1860}

Dispharagus mamillaris, Molin, 1860b: 499.

Dispharagus mamillaris, Drasche, 1883b: 210, Taf. XII,

fig. 27.

Dispharagus mammillaris (sic), Stossich, 1891: 95.

Acuaria mamilaris, Railliet, Henry \& Sissoff, 1912: 623.

Material: Corvus cajanus (Cyanocorax cayanus), $11+q$, Villa do Manaos, 23-vi-1834, inter tunicas ventriculi.

Remarks: Species inquirendae in agreement with Molin (1860b), who examined only females. As described by Molin (1860b), the cephalic cordons are anastomosing
(Syncuaria), but in the description of Drasche (1883b) and later writers, these are gradually disappearing, not recurrent or anastomosing (Acuaria).

Status: Without reviewing the original material, the species must be considered incertae sedis.

Ascaris marginata Rudolphi, 1809

Ascaris marginata, Rudolphi, 1809: 138.

Ascaris marginata, Diesing, 1851: 180.

Toxocara canis, Sprent, 1958: 184.

Material: Canis familiaris (Canis lupus), Junio; Canis azarae (Cerdocyon thous), Barra do Rio Jauru, Octobri; Rio Araguay, 15-xi-1823, in eorum intestinis tenuibus.

Status: In agreement with Sprent (1958) that this is a synonym of Toxocara canis (Werner, 1782) Stiles, 1905.

\section{Ascaris mascula Rudolphi, 1819}

Ascaris mascula, Rudolphi, 1819: 653.

Ascaris mascula, Diesing, 1851: 154.

Falcaustra mascula, Freitas \& Lent, 1941: 25.

Material: In intestinis Colubri n. 16 (Rudolphi). Coluber lichtensteinii (Mastigodryas bifossatus) in intestinis; Pseudophis bivittatus (Dendrophidion dendrophis ?), Martio (Diesing).

Status: Falcaustra mascula (Rudolphi, 1819) Freitas \& Lent, 1941.

Dochmius maxillaris Molin, 1861

Dochmius maxillaris, Molin, 1861a: 494.

Uncinaria maxillaris, Stossich, 1899: 126.

Dochmius maxillaris, Biocca, 1961b: 10.

Material: Procyon cancrivorus, 6 ก, 8 +, Ypanema, 30vi-1820, in intestino.

Remarks: Biocca (1961b) proposed this species as a synonym of Docmius bidens.

Status: Uncinaria maxillaris (Molin, 1861) Stossich 1899.

\section{Physaloptera maxillaris Molin, 1860}

Physaloptera maxillaris, Molin, 1860d: 645.

Physaloptera maxillaris, Drasche, 1882a: 128, Taf. VIII,

fig. 24 und 25.

Physaloptera maxillaris, Ortlepp, 1922: 1019.

Material: Mephitis chinche (Conepatus chinga), 51 ठे $\partial^{\lambda}$, 38 우, Borba, 9-v-1823, in ventriculo.

Status: Physaloptera maxillaris Molin, 1860.

Spiroptera mediospiralis Molin, 1860

Spiroptera mediospiralis, Molin, 1860a: 968.

Spiroptera mediospiralis, Drasche, 1883b: 203, Taf. XII, fig. 16.

Physocephalus mediospiralis, Hall, 1916: 219. 
Tejeraia mediospiralis, Diaz-Ungría, 1963: 901.

Material: Tapirus americanus (Tapirus terrestris), $15 \hat{\partial} \hat{\partial}$, 19 우오, Matogrosso, 21-iii-1829, in ventriculo; Dasyprocta aguti, several hundred $\hat{\partial} \sigma^{\lambda}$ and $q+$, Caiçara, Januario, in ventriculo.

Status: Tejeraia mediospiralis (Molin, 1860) Diaz-Ungría, 1963.

Cucullanus microcephalus Rudolphi, 1819

Cucullanus testudinis, Rudolphi, 1819: 21.

Cucullanus microcephalus, Dujardin, 1845: 246.

Cucullanus microcephalus, Diesing, 1851: 242.

Camallanus amazonicus, Ribeiro, 1941: 724.

Material: Rhinemys depressa (Phrynops sp.), Decembri, in ventriculo et intestinis tenuibus; Podocnemis tracaxa (Peltocephalus dumerilianus), Octobri; Podocnemis expansa Martio, in intestinis tenuibus.

Status: Camallanus amazonicus Ribeiro, 1941 (Cucullanus microcephalus Diesing, 1851, not C. microcephalus Dujardin, 1845.)

Ascaris microlabium Molin, 1860

Ascaris microlabium, Molin, 1860e: 336.

Ascaris microlabium, Diesing, 1861: 658.

Ascaris microlabium, Drasche,1882a: 134, Taf. IX, fig. 10-12.

Material: Falco coronatus (Harpyhaliaetus coronatus), 10

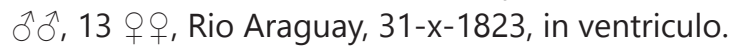

Remarks: After Drasche (1882a), the species disappears from the literature, although by the figures it looks like a Contracaecum.

Status: Until the original material is reviewed, it should be considered as incertae sedis.

\section{Trichocephalus minutus Rudolphi, 1819}

Trichocephalus minutus, Rudolphi, 1819: 638.

Trichocephalus minutus, Diesing, 1851: 293.

Trichuris minutus, Chandler, 1930: 203.

Material: Didelphis myosurus (Metachirus nudicaudatus), Majo; Didelphis domestica (Monodelphis domestica), Cuyaba, 4-iii-1824; Didelphis cancrivora (Didelphis marsupialis), Decembri; Didelphis azarae (Didelphis aurita), Borba, 28-vii-1830, in carum intestino coeco.

Status: Trichuris minuta (Rudolphi, 1819) Chandler, 1930.

Ascaris molini Stossich, 1896

Ascaris spiralis, Rudolphi, 1801: 25.

Ascaris spiralis, Molin, 1860e: 339.

Ascaris depressa, Rudolphi, 1819: 42.

Ascaris spiralis, Diesing, 1861: 667.
Ascaris molini, Stossich, 1896: 75.

Material: Picus comatus (Campephilus melanoleucus), 6 exemplars, Barra do Rio Janeiro, 8-x-1825.

Remarks: Stossich renames Ascaris spiralis of Molin (1860) because Rudolphi (1801) describes a species with the same name, but this same author synonymizes his species with Zeder's Ascaris depressa, and then homonymia does not exist and the new name is not justified.

Status: Nomen nudum.

Spiroptera molini Stossich, 1898

Spiroptera crassicauda, Creplin, 1829: 3.

Spiroptera crassicauda, Molin, 1860a: 937.

Spiroptera crassicauda, Drasche, 1883b: 197, Taf. XIII, fig. 16.

Spiroptera molini, Stossich, 1898: 100.

Material: Tinamus tao, Barra do Rio Negro, Octobri; Tinamus strigulosus (Crypturellus strigulosus), Pará, Decembri. Inter eorum tunicas ventriculi.

Remarks: Stossich (1898), without comments, renames the species because the name exists as Spiroptera crassicauda Creplin, 1829, but actually the species of Creplin is Streptocara crassicauda. Then the homonymy doesn't exist and the name molini is not necessary if the species Spiroptera crassicauda Molin, 1860 is not a Streptocara.

Status: Nomen nudum.

\section{Physaloptera monodens Molin, 1860}

Physaloptera monodens, Molin, 1860d: 658.

Physaloptera monodens, Drasche, 1882a: 127, Taf. VIII, fig. 8-10.

Physaloptera monodens, Ortlepp, 1922: 1030.

Material: Boa constrictor, $20 \hat{\partial} \delta, 52$ + $q$, Ypanema, 17ii-1820; 3 우, same host and locality, 6-ii-1819; $27 \hat{\jmath} \widehat{\delta}, 58$ 우오, same host and locality, 6-iii-1819; and 23-ii-1822, in ventriculo et intestino.

Status: Physaloptera monodens Molin, 1860.

Oesophagostomum monostichum (Diesing, 1851)

Sclerostomum monostichum, Diesing, 1851: 306.

Oesophagostomum monostichum, Molin 1861a: 448.

Neomurshidia monostichum, Chabaud, 1957: 116.

Material: Tapirus americanus (Tapirus terrestris), in intestino coeco, Aprili and Septembri (Diesing). Tapirus terrestris, several hundred $\hat{\partial} \delta^{\hat{~}}$ and $q+q_{1}$, Ytararè, 10-viii-1820; Barra do Rio Jaguaricata, 8-iii-1825; Matogrosso, iv-1829; Cachoeira do Bananeira, 1-ix-1929; same locality, 4-ix-1829; Forte do Rio Branco, 1935.

Status: Neomurshidia monosticha (Diesing, 1851) Chabaud, 1957. 
Filaria motacillae Rudolphi, 1819

Filaria motacillarum, Rudolphi, 1819: 9.

Filaria motacillae, Rudolphi, 1819: 635.

Diplotriaena motacillarum, Lopez-Neyra, 1956: 24.

Diplotriaena motacillae, López-Neyra, 1956: 24.

Material: In Muscicapae n. 144 (Rhynchocyclus olivaceus), Registro do Sai, Aprili, abdomine.

Status: Probably a Diplotriaena. Incertae sedis. See Filaria abbreviata.

Physaloptera mucronata Diesing, 1851

Physaloptera mucronata, Diesing, 1851: 235.

Physaloptera mucronata, Diesing, 1857: 16, Taf. II, fig. 17-28.

Physaloptera mucronata, Drasche, 1882a: 128.

Physaloptera mucronata, Ortlepp, 1922: 1103.

Material: Chamsa nigra (Melanosuchus niger), 400 exemplars, Borba, 27-vi-1830, in ventriculo.

Remarks: In agreement with Ortlepp (1922); this is not Physaloptera. Drasche points out that it is a synonym of Ascaris lanceolata Molin, 1860 (see this species), but figures in Diesing (1857) show 2 labia and strong caudal alae.

Status: Incertae sedis.

Diploodon mucronatum Molin, 1861

Diploodon mucronatum, Molin, 1861a: 474.

Diploodon mucronatum, Biocca, 1961a: 3, fig. 1-4.

Material: Dasypus gilvipes (Euphractus sexcinctus), 10 $\widehat{\jmath} \widehat{\jmath}, 3$ q 9 , Pouso dos Dois Irmãos, 23-vii-1825, in intestino tenui.

Status: Ancylostoma mucronatum (Molin, 1861) Biocca, 1961.

Kalicephalus mucronatus Molin, 1861

Kalicephalus mucronatus, Molin, 1861a: 547.

Kalicephalus mucronatus, Schad, 1962: 1052.

Material: Crotalus horridus (Crotalus durissus), 1 , , Cuyaba, 3-vi-1825; 7 गे ô, 20 우, Ypanema, 30-v-1819; 1 गे, 3 우오, Matogrosso, 29-v-1828, in intestino.

Status: Synonym of Kalicephalus c. costatus Molin, 1861 in Schad (1962).

Ascaris multilobata Drasche, 1882

Ascaris multilobata, Drasche, 1882b: 139, Taf. XII, fig. 4-6.

Material: Dicholophus marcgrafi (Cariama cristata), no more data.

Remarks: The species, described by Drasche (1882b) of material supposedly collected by Natterer, has not subsequently been cited. In his figure 4 there are interlabia that would be Contracaecum.
Status: Until the material is reviewed the species should be considered incertae sedis.

Filaria multipapilla Molin, 1858

Filaria multipapilla, Molin, 1858b: 385.

Breinlia brevicaudata Rodhain \& Vuylsteke, 1937: 225.

Oswaldofilaria brevicaudata, Freitas \& Lent, 1937: 440.

Filaria multipapilla (p.p.), Freitas \& Lent, 1937: 440.

Material: Thorictis dracaena (Dracaena guianensis), 10

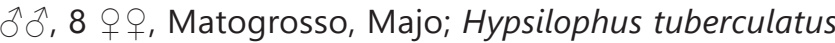
(Iguana iguana), 1 , Cuyaba, Augusto, in cavo abdominis.

Remarks: Rodhain and Vuylsteke (1937) described Breinlia brevicaudata of Iguana tuberculata from South America in the same year Freitas and Lent (1937) described some exemplars of the same host from Brazil and named them Oswaldofilaria brevicaudata (Rodhain \& Vuylsteke, 1937) n. comb. and allocated the $q$ of Molin from Iguana iguana in this species as Filaria multipapillata (p.p.).

Status: Until the material is reviewed the species should be considered species inquirenda.

Ascaris multipapillata Drasche, 1882

Ascaris multipapillata, Drasche, 1882b: 140, Taf. XII, fig. 7 und 8.

Contracaecum multipapillatum, Baylis 1920: 262.

Material: Tantalus loculator (Mycteria americana), no more data.

Status: Contracaecum multipapillatum (Drasche, 1882) Baylis, 1920.

Physaloptera murisbrasiliensis Diesing, 1861

Spiroptera bilabiata, Molin, 1860a: 928.

Physaloptera Muris brasiliensis, Diesing, 1861: 653.

Physaloptera murisbrasiliensis, Drasche, 1882a: 128,

Taf. VIII, fig. 11 und 12.

Physaloptera murisbrasiliensis, Ortlepp, 1922: 1023.

Material: Mus brasiliensis (Holochilus brasiliensis), 3 तิ $\widehat{0}$, 11 우, Ypanema, 19-ii-1819, in ventriculo.

Remarks: Diesing (1861) species inquirenda.

Status: Physaloptera murisbrasiliensis Diesing, 1861.

Filaria muscicapae coronatae Molin, 1858

Filaria muscicapae coronatae, Molin, 1858b: 434.

Filaria muscicapae coronatae, Yamaguti, 1961: 330.

Material: Muscicapa coronata (Onychorhynchus coronatus), $3 \hat{\partial} \hat{\jmath}$, Matogrosso, Julio, in cavo abdominis.

Remarks: Must be Strongyloidea in agreement with Molin (1858b), and to Yamaguti (1961) is incertae sedis.

Status: Should be considered species inquirenda until review of the material. 
Filaria myotherae campanisinae Molin, 1858

Filaria myotherae campanisinae, Molin, 1858b: 425.

Filaria myotherae campanisonae, Stossich, 1898: 74.

Material: Myothera campanisona (Chamaeza campanisona), 1 \&, Ypanema, Martio, ad oculum.

Remarks: Species inquirenda in agreement with Molin (1858b), who studied only one female.

Status: It should be considered species inquirenda until review of the material.

\section{Filaria myotherae chrysopygae Molin, 1858}

Filaria myotherae chrysopygae, Molin, 1858b: 425.

Diplotriaena myotherae chrysopygae, López-Neyra, 1956: 24.

Material: Myothera chrysopyga (Drymophila malura), 1 $\lambda, 2$ 우, Ypanema, Aprili, sub cute prope oculum.

Remarks: Molin (1858b) studied 3 exemplars that seemed to be Filaria abbreviata but decided they were too opaque to see the internal morphology and included them in species inquirenda.

Status: Based on this similarity noted by Molin (1858b) with Filaria abbreviata, some authors consider it as Diplotriaena but the localization, the eye, makes it unlikely. Until reviewed the material must remain species inquirenda or nomen dubium.

Filaria myotherae regis Molin, 1858

Filaria myotherae regis, Molin, 1858b: 424.

Material: Myothera (Turdus) rex (Grallaria varia), 1 , Marabitanas, Majo, ad renes.

Remarks: In agreement with Molin (1858b), who studied only one female.

Status: This species has been ignored by authors and should be species inquirenda until review of the exemplar is done.

Filaria myotherae ruficipitis Molin, 1858

Filaria myotherae ruficipitis, Molin, 1858b: 424.

Filaria myotherae ruficipitis, Stossich, 1898: 74.

Material: Myothera ruficeps (Formicarius colma), 3 우, Marabitanas, Majo, in cavo abdominis.

Remarks: Species inquirenda in agreement with Molin (1858b).

Status: This species has been ignored by authors and should be species inquirenda.

Filaria nodosa Molin, 1858

Filaria nodosa, Molin, 1858b: 380, Taf. I, fig. 3.

Filaria nodosa, Webber, 1955: 239.

Material: Many females in Jacchus (Hapale) melanurus (Mico melanurus), Matogrosso, Decembri, sub cute ad scapulas et inter musculus pectorales; Cebus personatus (Callicebus personatus), Irisanga, Decembri, inter cutem et musculus ad costas.

Status: The species has been overlooked by other authors and could be a species of Mansonella (Tetrapetalonema), but until the material is studied, it should be considered incertae sedis.

Trichocephalus nodosus Rudolphi, 1809

Trichocephalus muris, Goeze, 1782: 119.

Trichocephalus nodosus, Rudolphi, 1809: 96.

Trichocephalus nodosus, Diesing, 1851: 293.

Trichuris muris, Hall, 1916: 26.

Material: Holochilus brasiliensis, Septembri; Hesperomys leucogaster (Holochilus brasiliensis), Julio; Isothrix pachyura (Isothrix b. bistriata), Junio; Isothrix bistriata, Julio. In eorum intestino coeco.

Status: Synonym of Trichuris muris (Schrank, 1788) Hall, 1916.

Spiroptera nuda Molin, 1860

Spiroptera nuda, Molin, 1860a: 919.

Spiroptera nuda, Drasche, 1883b: 193.

Spiroptera nuda, Stossich, 1898: 89.

Material: Rallus mangle (Aramides mangle), $1 \hat{\delta}$, Sapitiba, Februario, in intestinis.

Remarks: The species was described from two species of Old World Falconidae and a Rallidae from Brazil. Possibly there are two different species.

Status: The material collected by Natterer in Brazil was a single male. Until the species is revised, it should be considered incertae sedis.

\section{Oxyuris obesa Diesing, 1851}

Oxyuris obesa, Diesing, 1851: 141.

Oxyuris obesa, Diesing, 1857: 12, Taf. I, fig. 1-6.

Oxyuris obesa, Drasche, 1882a: 118.

Protozoophaga obesa, Travassos, 1923: 36.

Material: Hydrochoerus capybara (Hydrochoerus hydrochaeris), many exemplars, Ypanema, 16-ii-1819, in intestino coeco.

Status: Protozoophaga obesa (Diesing, 1851) Travassos, 1923.

Filaria obtusa Rudolphi, 1802

Filaria obtusa, Rudolphi, 1802: 3.

Filaria obtusa, Diesing, 1851: 267.

Filaria obtusa, Molin, 1858b: 397.

Filaria obtusa, Stossich, 1898: 27.

Diplotriaena obtusa (Rudolphi, 1802), Henry \& O'zoux, 1909: 547. 
Material: Hirundo purpurea (Progne subis), Barra do Rio Negro, December, in eorum cavo abdominis; Hirundo versicolor (Tachycineta leucorrhoa), $7 \hat{0} \hat{0}, 7$ 우, Matogrosso, Junio; Myothera caudacuta (Culicivora caudacuta), 1 , Marabitanas, April; Xenops sp. (Cercomacra nigrescens), 2

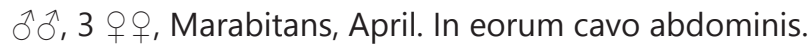

Status: Diplotriaena obtusa (Rudolphi, 1802) Henry \& O'zoux, 1909.

Spiroptera obtusa Rudolphi, 1819

Ascaris teres, Goeze, 1782: 86 (in partim). Lumbricus muris, Werner, 1782: 10.

Ascaris muris, Gmelin, 1790: 3032.

Ascaris obtusa, Frölich, 1791: 88.

Fusaria muris, Zeder, 1803: 106.

Spiroptera obtusa, Rudolphi, 1819: 27, 249.

Spiroptera obtusa, Diesing, 1851: 214.

Spiroptera obtusa, Molin, 1860a: 934.

Protospirura muris, Seurat, 1915: 565.

Material: Mus decumanus (Mus musculus), in Brasilia (probably Rio de Janeiro).

Status: Protospirura muris (Goeze,1790) Seurat, 1915.

Physaloptera obtusissima Molin, 1860

Physaloptera obtusissima, Molin, 1860d: 654.

Physaloptera obtusissima, Drasche, 1882a: 127, Taf.

VIII, fig. 13 und 14.

Physaloptera obtusisima, Ortlepp, 1922: 1032.

Material: Ophis coeruleus (?), $16 \widehat{\partial} \hat{\partial}, 17 q q+$, Cuyaba,

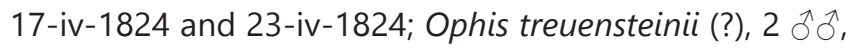
5 우우, Ypanema, 18-viii-1822; same host, 6 d $\delta$ d, 13 우우, Caiçara, 16-i-1826; Ophis saurocephalus (Xenodon severus), 3 సर 1 , 1 q, Matogrosso, 23-i-1829; same host, Caiçara, 20vi-1826; same host, 11 के ठै, 28 우우, Cuyaba, 27-ii-1825; Ophis rhodogaster (?), 7 ๙ิ่, 18 우, Cuyaba, 7-vi-1825; Pseudophis cinerascens (?), 25 $\widehat{\partial}, 57$ 우우, Cuyaba, 11-iii1824, 26-iv-1824, and 15-xi-1824; Lygophis regius (?), 1 ô,

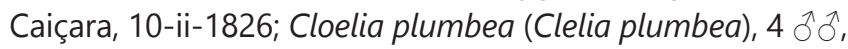
8 우우, Cuyaba, 15-iv-1824; same host and locality, 28 수의, 45 우, 15-xi-1824 and 8-v-1824; Cloelia fasciata (Anilus scitale ?), 1 q, Cuyaba, 23-iv-1825; Erythrolamprus venustissimus (Erythrolamprus aesculapii), $2 \stackrel{\AA}{\delta}$, Cuyaba, 17-iv-1825; Spilotes pullatus, 3 ồ $\hat{0}, 9$ 우, Forte do Rio Branco, 7-iv1832; Bothrops jararacca (Bothrops jararaca), 2 ठ $\delta$, Cuyaba, 22-iv-1824, in ventriculo et in intestinis.

Status: Physaloptera obtusissima Molin, 1860.

Filaria obtuso-caudata Rudolphi, 1819

Filaria obtuso-caudata, Rudolphi, 1819: 634.

Filaria obtuso-caudata, Dujardin, 1845: 55.

Filaria obtuso-caudata, Diesing, 1851: 277.
Filaria obtuso-caudata, Molin, 1858b: 413.

Monopetalonema obtuse-caudatum, Diesing, 1861: 710.

Filaria obtusocaudata, Stossich, 1898: 37.

Diplotriaena obtusocaudata, Walton, 1927: 120.

Diplotriaena obtusocaudata, Anderson, 1959: 261.

Diplotriaena obtusocaudata, Hartwich, 1966: 149.

Material: Picus lineatus (Dryocopus lineatus), in cavo thoracis (Rudolphi) (lost Hartwich, 1966); Picus flavescens (Celeus flavescens), $6 \hat{o} \hat{d}, 10$ q $q$, Ypanema, Majo, sub cute colli; same host, Rio Muria, Februario, inter tunicas oesophagi; same host, Borba, Majo, sub cute colli et inter tunicas oesophagi; same host, Ypanema, Aprili, sub cute in regione superorbitali; Picus robustus (Campephilus robustus) (lost) inter musculus colli; Picus passerinus (Veniliornis passerinus), Rio Negro apud Thomas, Decembri, ad gutturem sub cute; Picus aurulentus (Piculus aurulentus), Ypanema, Junio, sub cute et inter musculos colli; Picus leucolaemus (Piculus leucolaemus), 1 d, 1 \&, Egenho do Gama, Julio, sub tela cellulosa; Picus jumana (Celeus elegans), Rio Negro apud Thomas, Decembri, ad gutturem sub cute; same host, Borba, Februario, in tumore colli; Picus rubricollis (Campepephilus rubricollis), Pará, Decembri, inter musculos colli; Perdix leucostricta (Odontophorus stellatus), $2 \hat{\jmath} \hat{0}, 4$ q + , Junio, Borba, inter musculos alarum.

Status: Uncertain. Walton (1927) identified fragments of a filarioid found as Diplotriaena obtusocaudata, but Anderson (1959) recommended that the name be removed from the list of known species of Diplotriaena; thus, it is incertae sedis.

\section{Elaphocephalus octocornutus Molin, 1860}

Elaphocephalus octocornutus, Molin, 1860e: 344.

Elaphocephalus octocornutus, Diesing, 1861: 670.

Elaphocephalus octocornutus, Drasche, 1883a: 113, Taf. III, fig. 21-23.

Material: Psittacus macao (Ara macao), 1 q, Ponte do Guaporé, 28-ix-1827, ad origenem digitorum pedis.

Remarks: The species was described based on a female (Molin, 1860e) in very good condition; it looks like a Schistophorinae or close to Sobolevicephalus (= ? Hadjelia), though its location has been considered a filaria (Yorke \& Maplestone, 1926). It has not been found subsequently.

Status: Without reviewing the original material, the species must be considered incertae sedis.

Ascaris obvelata Rudolphi, 1802

Ascaris obvelata, Rudolphi, 1802: 2.

Oxyuris obvelata, Bremser in Rudolphi, 1819: 19.

Ascaris oxyura, Nitzsch, 1821: 48.

Ascaris oxyura, Diesing, 1851: 145.

Syphacia obvelata, Seurat, 1916: 65. 
Material: Mus musculus, Septembri; Holochilus brasiliensis, Ypanema, Septembri; Hesperomys leucogaster (Holochilus brasiliensis), Ypanema, Junio; Oxymycterus nasutus, Ypanema, Januario.

Remarks: The species is a parasite of Mus musculus; other samples would be another species of Syphacia.

Status: Syphacia obvelata (Rudolphi, 1802) Seurat, 1916.

\section{Ascaris pacheia Drasche, 1882}

Ascaris pacheia, Drasche, 1882b: 140, Taf. XII, fig. 9.

Ascaris pacheia, Stossich, 1896: 27.

Material: Sarcorhamphus papa, no more data.

Status: The species disappears from the literature after Stossich's publication, so until there is a review of the material, it should be considered species inquirenda or nome oblitum.

\section{Oesophagostomum pachycephalum Molin, 1861}

Oesophagostomum pachycephalum, Molin 1861a: 450.

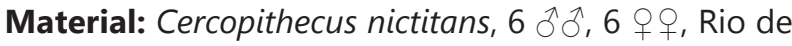
janeiro, 8-iii-1821.

Remarks: It is the only species of Oesophagostomum described in the New World; the host should have been a pet.

Status: Oesophagostomum pachycephalum Molin, 1861.

\section{Filaria papillicauda Molin, 1858}

Filaria papillicauda, Molin, 1858b: 380.

Filaria immitis, Stossich, 1898: 34.

Dirofilaria immitis, Railliet \& Henry, 1911a: 386.

Material: Canis brachyuris (Chrysocyon brachyuris), Rio Araguay, $20-x-1823$, inter fibras musculares cordis; Rio Araguay, $21-x-1823$, in cavo abdominis.

Remarks: Molin (1858b) had only females to describe the species, but see $F$. canis brachyuri.

Status: Dirofilaria immitis (Leidy, 1856) Railliet \& Henry, 1911.

\section{Ascaris papillosa Molin, 1860}

Ascaris papillosa, Molin, 1860e: 338.

Ascaris papillosa, Diesing, 1861: 658.

Ascaris papillosa, Drasche, 1882a: 129, Taf. IX, fig. 24.

Heterakis papillosa, Stossich, 1888: 291, Tav. VII, fig. 24.

Subulura (?) papillosa, Railliet \& Henry, 1914: 680.

Ascaris papillosa, Inglis, 1958: 600.

Subulura papillosa, Vicente et al., 1996: 6.

Material: Corvus cajanus (Cyanocorax cayanus), 1 है, Barra do Rio Negro, 2-x-1830, in intestino.

Status: Subulura papillosa (Molin, 1860) Railliet \& Henry, 1914 or incertae sedis, Inglis (1958).
Spiroptera papillosa Molin, 1860

Spiroptera papillosa, Molin, 1860a: 929.

Spiroptera papillosa, Drasche, 1883b: 195, Taf. XII, fig.

20; Taf. XIII, fig. 7.

Oxyspirura (?) papillosa, Ramson, 1904: 27.

Thelazia papillosa, Railliet \& Henry, 1910a: 216.

Thelazia (Thelazia) papillosa, Travassos, 1918b: 222.

Thelazia papillosa, Anderson \& Diaz-Ungría, 1959: 70.

Material: Falco destructor (Harpia harpyja), 6 q+, Barra do Rio Negro, 22-iii-1834; 5 오, same host and locality, 18v-1833; Falco gracilis (Geranospiza caerulescens), 2 ôे 1 +, Pausecco, 2-vi-1826. Sub eorum membrana nictitante.

Remarks: Travassos believed it is probably identical to Thelazia campanula, but Anderson \& Diaz-Ungría (1959) disagree.

Status: Thelazia papillosa (Molin, 1860) Railliet \& Henry, 1910.

Filaria papilloso-annulata Molin, 1858

Filaria papilloso-annulata, Molin, 1858b: 399.

Material: Falco swainsonii (Gampsonyx swainsonii), 2 우, Caiçara, May, in regione postica cavi orbitae inter musculus. There are only two females of this species.

Status: This species has been ignored by authors and should be species inquirenda until review of the material.

Eustrongylus papillosus (Rudolphi, 1802)

Strongylus papillosus, Rudolphi, 1802: 42.

Eustrongylus papillosus, Diesing, 1851: 326.

Hystrichis papillosus, Molin, 1861a: 607.

Eustrongyloides papillosus, Jägerskiöld, 1909: 17.

Eustrongyloides ignotus, Jägerskiöld, 1909: 20.

Eustrongyloides perpapillatus, Jägerskiöld, 1909: 26.

Material: Ardea leuce (Ardea herodias), Februario; Ardea cocoi, Octobri et Decembri; Plotus anhinga (Anhinga

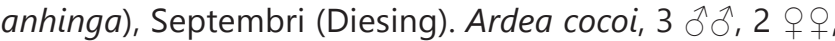
Matogrosso, 19-xii-1828; same host, 1 j, 3 q + , Caiçara, 25-x-1825, inter tunicas ventriculi; Ardea pinnata (Botaurus pinnatus), 1 , Araguay, Registo do Rio Grande, 30-x-1823, ad ventriculum capsula inclusus; Plotus melanogaster ( $A n-$ hinga anhinga), 1 ภ, 2 우, Matogrosso, 20-viii-1828; Tantalus loculator (Mycteria americana), 11 q + , Porto do Rio Parana, 22-v-1823, inter eorum tunicas ventriculi (Molin).

Remarks: Jägerskiöld (1909) restricts Eustrongyloides papillosus to Europe and described exemplars from Ardea cocoi, Anhinga anhinga, and Botaurus pinnatus as Eustrongyloides ignotus and exemplars from Ardea herodias as Eustrongyloides perpapillatus.

Status: Eustrongylides ignotus Jägerskiöld, 1909 and Eustrongylides perpapillatus Jägerskiöld, 1909 as Eustrongylides papillosus (p.p.) (Rudolphi, 1802) Jägerskiöld, 1909. 
Physaloptera papillotruncata Molin, 1860

Physaloptera papillotruncata, Molin, 1860d: 647.

Physaloptera papillotruncata, Drasche, 1882a: 127, Taf.

VIII, fig. 21-23.

Physaloptera papillotruncata, Ortlepp, 1922: 1016.

Material: Myrmecophaga jubata (Myrmecophaga tridactyla), 640 of $\hat{d}$ and $+9+$, Matogrosso, 3-vii-1828; same host, 296 수 $\hat{o}$ and 우우, Caiçara, 13-x-1825; same host, 3 exemplars, Ytararé, 21-i-1821; same host, 3 exemplars, Rio des Pedras, 18-iv-1823; same host, 19 exemplars, Cuyaba, 1-ii1824; same host, 63 exemplars, Barra do Rio Jauru, 10-x1825; same host, many exemplars, Egenho do Cap Gama, 9-ix-1826; Myrmecophaga didactyla (Cyclopes didactylus),

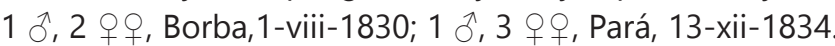
In eorum ventriculo.

Status: Physaloptera papillotruncata Molin, 1860

\section{Tropidocerca paradoxa (Diesing, 1835)}

Tropisurus paradoxus, Diesing, 1835: 94, Tab. I und II.

Tropidocerca paradoxa, Diesing, 1851: 207.

Tropidocerca paradoxa, Diesing, 1861: 673.

Tropidocerca paradoxa, Drasche, 1883a: 114, Taf. III, fig. 25 und 26.

Tetrameres paradoxa, Travassos, 1914b: 151.

Material: Cathartes urubu (Cathartes burrovianus), Irisanga, Decembri; Forte do Rio Branco, Aprili, 1832, inter musculos et tunicas ventriculi; Strix torquata (Pulsatrix perspicillata), Para, Novembri, inter tunicas proventriculi.

Status: Tetrameres paradoxus (Diesing, 1835) Travassos, 1914

\section{Spiroptera penihamata Molin, 1860}

Spiroptera penihamata, Molin, 1860a: 938

Spiroptera penihamata, Drasche, 1883b: 198, Taf. XIII, fig. 6. Spiroptera penihamata, Stossich, 1898: 100.

Spiroptera penihamata, Cram, 1926: 205.

Streptocara penihamata, Yamaguti, 1961: 264.

Streptocara penihamata, Vicente et al., 1996: 62.

Material: Strix atricapilla (Megascops atricapilla), 4

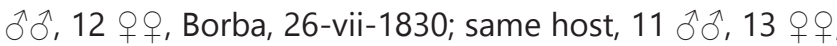
Egenho do Cap Gama, 28-ix-1826, inter tunicas ventriculi; Strix flammea (Asio flammeus), 5 के $\hat{0}, 10$ 우, Ypanema, 13x-1821 and 17-viii-1822, inter plicas ventriculi; Strix griseata (Lophostrix cristata), 8 $\precsim$, 16 우, Borba, 22-vii-1830; Strix albomarginata (Strix huhula), 1 , Borba, 2-viii-1830, inter earum tunicas ventriculi.

Remarks: The species has been identified as Streptocara, but Gibson (1968) in his review wrote: "This species does not belong in Streptocara, but so little information about it is available that it cannot be transferred readily to a known genus."
Status: Until review of the original material is done, this should be considered incertae sedis.

Filaria perdicis dentatae Molin, 1858

Filaria perdicis dentatae, Molin, 1858b: 427.

Material: Perdix dentata (Odontophorus capueira), in cavo abdominis.

Remarks: Species inquirenda in agreement with Molin (1858b), who wrote: "I had the opportunity to examine only one but a very opaque specimen of this kind, but I believe that it is a strongylid and not a filaria."

Status: Until review of the original material is done, this should be considered nomen nudum.

Filaria perforans Molin, 1858

Filaria martis, Gmelin, 1790: 3040.

Filaria perforans, Molin, 1858b: 387.

Filaria perforans, Hall, 1916: 180.

Material: Gulo barbatus (Eira barbara), Ypanema, Decembri, in cavo thoracis.

Status: Synonym of Filaria martis Gmelin, 1790 in Hall (1916)

Monopetalonema physalura Bremser in Diesing, 1851

Filaria alcedinis, Rudolphi, 1819: 635.

Filaria physalura, Bremser in Diesing, 1851: 276.

Filaria physalura, Molin, 1858b: 412.

Monopetalonema physalurum, Diesing, 1861: 710.

Filaria physalura, Stossich, 1898: 76.

Monopetalonema alcedinis, Freitas \& Lent, 1936: 752.

Monopetalonema alcedinis, Vicente et al., 1996: 95.

Material: Alcedinis n. 82 (Megaceryle torquatus), Brasilia (Rudolphi). Alcedinis superciliosa (Chloroceryle aenea), Octobri, cavo thoracis et abdomen.; Alcedinis torquata (Megaceryle torquatus), Curytiba, Octobri, in cavo thoracis ad cor; in cavo abdominis. Inter intestinis; same host, Ypanema, Junio, in regione coccigea inter musculus et cutem; Alcedinis amazona (Chloroceryle amazona), Ytararé, Martio; same host, Irisanga, Martio; same host, Mato dentro (sic), Decembri, in eorum cavo abdominis (Molin).

Status: Monopetalonema alcedinis (Rudolphi, 1819) Freitas \& Lent (1936).

Ancyracanthus pinnatifidus Diesing, 1838

Ancyracanthus pectinatus, Diesing, 1838: 189.

Ancyracanthus pinnatifidus, Diesing, 1839: 227, Taf.

XVIII, fig. 1-20.

Ancyracanthus pinnatifidus, Diesing, 1851: 208.

Ancyracanthus pinnatifidus, Drasche, 1883a: 111, Taf. IV, fig. 6-11.

Material: Podocnemis expansa, Borba, Junio, Augusto, 
in intestinis tenuibus; same host, Forte do Rio Branco, Majo, Augusto; Podocnemis tracaxa (Peltocephalus dumerilianus), Barra do Rio Negro, Octobri, in ventriculo et processibus ventriculi.

Status: Ancyracanthus pinnatifidus Diesing, 1839.

Filaria piprae caudatae Molin, 1858

Filaria piprae caudatae, Molin, 1858b: 424.

Filaria piprae caudatae, Stossich, 1898: 76.

Material: Pipra caudata (Chiroxiphia caudata), in cavo abdominis, externe ad intestina.

Remarks: In the Natterer collection, Molin (1858b) found only one very opaque examplar, and he could not determine the sex or other characteristic (a larva?). He placed it as species inquirendae.

Status: Must be regarded as nomen nudum.

Filaria pistillaris Molin, 1858

Filaria pistillaris, Molin, 1858b: 381.

Filaria pistillaris, Stossich, 1898: 76.

Filaria pistillaris, Yamaguti, 1961: 640.

Material: Sciurus igniventris, $4 q q$, Marabitans, Januario, sub cute abdominis.

Remarks: Tentatively assignated to Filaria by Yamaguti (1961), but according to the original description and the subcutaneous location may be Mansonella (Tetrapetalonema).

Status: Until reviewed, the material must remain incertae sedis.

\section{Spiroptera pistillaris Molin, 1860}

Spiroptera pistillaris, Molin, 1860a: 955.

Spiroptera pistillaris, Drasche, 1883b: 201, Taf. XIII, fig.

17.

Pelecitus circularis, Railliet and Henry, 1910b: 251.

"Spiroptera" pistillaris, Yamaguti, 1961: 330.

Material: Strix magallanica (Bubo virginianus), $13 \hat{\jmath}$, 14 q, , Matogrosso, 25-x-1828, in cystide ad tendinem Achillis.

Remarks: In agreement with Drasche (1883b) that it is identical to Spiroptera circularis, and it was followed by subsequent authors.

Status: Synonym of Pelecitus circularis (Molin, 1860) Railliet \& Henry, 1910.

\section{Filaria podinemae sciptae Molin, 1858}

Filaria podinemae sciptae, Molin, 1858b: 430.

Material: Podinema scipta (sic) (?), Matogrosso, November, in adipe cavi abdom.

Remarks: Species inquirendae in agreement with Molin (1858b), who had the opportunity to examine only one, but it was a too poorly preserved specimen.

Status: Must be considered as nomen nudum.

Filaria podoae Molin, 1858

Filaria podae, Molin, 1858b: 428.

Material: Podoa surinamensis (Heliornis fulica), Matogrosso, Septembri, sub cute eolli.

Remarks: Molin (1858b) had a single exemplar in very poor condition from which he could not determine any characteristic, so the species was assigned as inquirendae.

Status: Must be regarded as nomen nudum.

Spiroptera posthelica Molin, 1860

Spiroptera posthelica, Molin, 1860a: 926.

Cheilospirura posthelica, Diesing, 1861: 684.

Cheilospirura posthelica, Drasche, 1883b: 210, Taf. XII,

fig. 26; Taf. XIII, fig. 20.

Spiroptera posthelica, Stossich, 1898: 86.

Acuaria posthelica, Yamaguti, 1961: 268.

Material: Timamous tao, $3 \hat{\jmath} \widehat{\jmath}$, Barra do Rio Negro, 7-x1830, inter tunicas ventriculi.

Remarks: Drasche (1883b) points out the similarity of this species with Spiroptera helicina (Pelecitus helicina), which could be as the caudal end of male; however, Yamaguti (1961) includes it among the Acuaria species, possibly by having the spicules short and equal, and its location in the crop. Both cases are indexed as possible, but without reviewing the original material, it should be considered as incertae sedis.

Status: Species incertae sedis.

Physaloptera praeputialis von Linstow, 1888

Physaloptera terdentata, Molin, 1860: 651 (in partim).

Physaloptera praeputialis, von Linstow, 1888: 238, fig.

9 and 10.

Physaloptera praeputialis, Ortlepp, 1922: 1043.

Material: Felis tigrina (Leopardus tigrinus), 2 우, no more data, in corum ventriculo.

Remarks: See Physaloptera terdentata.

Status: Physaloptera praeputialis von Linstow, 1888.

Spiroptera pulchella Molin, 1860

Spiroptera pulchella, Molin, 1860a: 985.

Spiroptera pulchella, Drasche, 1883b: 207.

Spiroptera pulchella, Stossich, 1898: 109.

Material: Rallus cayennensis (Aramides cajaneus), 18 오, Paranagoa, 14-xii-1820, inter tunicas ventticuli.

Remarks: Molin (1860a) placed the species as inquirenda; he had only females in good condition but was not able to observe a male.

Status: May be regarded as incertae sedis. 
Spiroptera quadrialata Molin, 1860

Spiroptera quadrialata, Molin,1860a: 965.

Spiroptera quadrialata, Drasche,1883b: 203.

Spiroptera quadrialata, Stossich, 1898: 89.

Spiroptera quadrialata, Hall, 1916: 221.

Spiroptera quadrialata, Yamaguti, 1960: 679.

Material: Mus musculus, 2 우, Rio de Janeiro, in ventriculo.

Remarks: Hall (1916) points out "the structure of the tail and position of the anus suggest Oxyuris and, in view of the alae, this worm is probably Oxyurls tetraptera, erroneously reported from the stomach of its customary host. The record as to the position of the vulva is probably due to an error in observation." I agree with the original description: the vulva has two lips, surrounded by papillae, and is in the posterior end. This description certainly matches a male of Aspiculuris.

Status: Aspiculuris quadrialata (Molin, 1860) n. comb.

Spiroptera quadricostata Molin, 1860

Spiroptera quadricostata, Molin, 1860a: 927.

Cheilospirura quadricostata, Diesing, 1861: 684.

Spiroptera quadricostata, Stossich, 1898: 118.

"Spiroptera" quadricostata, Yamaguti, 1961: 330.

Material: Psittacus aestivus (Amazona aestiva), $6 \hat{\partial} \hat{\delta}, 1$ †, Murungaba, April, inter musculos tibiae.

Remarks: The most outstanding characteristics are the cuadal alae supported by four papillae and the vulva near the apical extreme.

Status: Incertae sedis, perhaps a Pelecitus.

\section{Spiroptera quadridentata Molin, 1860}

Spiroptera quadridentata, Molin, 1860a: 941.

Spiroptera quadridentata, Drasche, 1883b: 199.

Spiroptera quadridentata, Stossich, 1898: 107.

Material: Alcedo americana (Chloroceryle americana), 1 ¡, 2 우, Praja de Cujutuba, inter tunicas ventriculi.

Status: The species has been overlooked by modern authors. Until the original material is reviewed, this should be considered incertae sedis.

\section{Diploodon quadridentatum Molin, 1861}

Diploodon quadridentatum, Molin, 1861a: 475.

Ancylostoma mycetis, Yorke \& Maplestone, 1926: 93.

Material: Mycetes coraya (Alouatta coraya), 1 , Cuyaba, 8-ii-1825

Remarks: Yorke and Maplestone (1926) renamed the species as Ancylostoma mycetis (homonym) with Strongylus quadridentatus Siebol, 1851 as a synonym of Ancylostoma duodenale, but the homonym does not exist.
Status: Ancylostoma quadridentatum (Molin, 1861) n. comb.

Filaria quadrilabiata Molin, 1858

Filaria quadrilabiata, Molin, 1858b: 417.

Tetracheilonema quadrilabiatum, Diesing, 1861: 711.

Filaria quadrilabiata, Stossich, 1898: 77.

Tetracheilonema quadrilabiatum, Vicente et al., 1996: 89.

Material: Tinamus rufescens (Rhychotus rufescens), 3 oj 12 오, Caiçara, Novembri, in cavo abdominis; Tinamus maculosus (Nothura maculosa), no more data, sub cute colli.

Status: Tetracheilonema quadrilabiatum (Molin, 1858) Diesing, 1861.

\section{Spiroptera quadrilabiata Drasche, 1883}

Spiroptera quadrilabiata, Drasche, 1883b: 214, Taf. XI, fig. 7 und 17; Taf. XIII, fig. 8.

Material: Dicotyles labiatus (Tayassu pecari), Brycon falcatus (Acestrorhynchus falcatus), and Trogon collaris, no more data.

Remarks: Drasche (1883b) wrote " $2 \hat{\partial} \bar{\alpha}$ and 1 a and 1 $\checkmark$ were in the bottles of Spiroptera strongylina and Spiroptera cesticillus from Dicotyles labiatus, 1 q with Spiroptera acuminata from Brycon falcatus and $1 \hat{\delta}$ with Spiroptera semilunaris from Trogon collaris." Spiroptera strongylina was collected in Dicotyles labiatus in April in Brasilia (Drasche (1833b). The only Dicotyles collected in April was in Registro do Sai (Pelzeln 1883) in 1818 (Vanzolini, 1993) along with Spiroptera cesticillus in the same host in Caiçara, Februari 11, 1826; Spiroptera acuminata in Brycon falcatus from Matogrosso, 10-xi-1826; and Spiroptera semilunaris in Trogon collaris in Villa Maria, 28-viii-1825 (Molin, 1860b). It means the hosts are mammals, a fish, and a bird, respectively. Localities are several too. So, the status of this species is very doubtful, but the figures show a Habronematidae.

Status: Nomen nudum.

\section{Spiroptera quadripapillosa Molin, 1860}

Spiroptera quadripapillosa, Molin, 1860a: 964.

Spiroptera quadripapillosa, Drasche, 1883b: 202.

Pelecitus quadripapillosus, Railliet \& Henry, 1910b: 251.

Material: Platalea ajaja, 3 우, Villa Maria, 7-viii-1825, inter tendines digiti pedis.

Status: Pelecitus quadripapillosus (Molin, 1860) Railliet \& Henry, 1910.

Spiroptera quadrispinosa Molin, 1860

Spiroptera quadrispinosa, Molin, 1860a: 987. 
Material: Anas dominica (Oxyura dominica), 7 우 Caiçara, 15-i-1826, inter tunicas ventriculi.

Remarks: Species inquirenda in agreement with Molin. He believed that "the armor of the head and shape of the eggs is not proper of the genus Spiroptera but is probably a Heteracis." The species is known only from the original description and perhaps is a Schistorophinae.

Status: Until the original material is reviewed, this should be considered incertae sedis.

Filaria quadriverrucosa Molin, 1858

Filaria quadriverrucosa, Molin, 1858b: 398.

Diplotriaena quadriverrucosa, Henry \& O'zoux, 1909: 547.

Material: Dendrocolaptes sp. (Xiphorhynchus elegans), $9 \hat{\partial} \hat{D}^{2}, 11$ 우, Egenho do Cap Gama, Julio; same host, Barra do Rio Negro, Octobri; Dendrocolaptes picus (Dendroplex picus), 1 q, Egenho do Cap Gama, Julio; Dendrocolaptes rufirostris (Campylorhamphus trochilirostris), Cuyaba, Augusto. In eorum cavo abdominis.

Status: Diplotriaena quadriverrucosa (Molin, 1858) Henry \& O'zoux, 1909, but in doubtful status (Anderson, 1959).

Spiroptera ralli Rudolphi, 1819

Spiroptera ralli, Rudolphi, 1819: 642.

Spiroptera ralli, Diesing, 1851: 228.

Spiroptera ralli, Molin, 1860a: 986.

Spiroptera ralli, Stossich, 1898: 118.

Material: Ralli $\mathrm{n} .180$ (Aramides mangle), inter tunicas ventriculi in Brasilia (Rudolphi). Rallus mangle (Aramides mangle) and Rallus saracura (Aramides saracura), inter tunicas ventriculi (Diesing). Rallus saracura (Aramides saracura), 1 , inter tunicas ventriculi (Molin).

Remarks: Species inquirenda in agreement with Diesing and Molin, who could see only a female in poor condition. Ralli n. 180 is Aramides mangle collected in Sapitiba in February 1818 (Pelzeln, 1871).

Status: If there was only a poor female in an unknown locality, this must be a nomen nudum.

Filaria ranae Molin, 1858

Filaria ranae, Molin, 1858b: 431.

Filaria ranae, Stossich, 1898: 78.

Foleyella ranae, Travassos, 1929a: 957.

Ochoterenella ranae, Esslinger, 1986: 215.

Material: Hypsiboas faber (?), 1 exemplar, Ypanema, Martio, in intestino.

Remarks: Species inquirendae in agreement with Molin (1858b).
Status: Molin (1858b) was able to examine only a poorly preserved specimen and doubted that it was really a filarid, but the species has been synonymized with Foleyella convoluta (Travassos, 1929a) and with Ochoterenella convoluta (Esslinger, 1986). After Molin's comments, it should be regarded as nomen nudum.

Ascaris reclinata Rudolphi, 1819

Ascaris reclinata, Rudolphi, 1819: 657.

Ascaris reclinata, Diesing, 1851: 174.

Subulura reclinata, Barreto, 1919: 11.

Subulura reclinata, Hartwich, 1965: 126.

Material: Crotophaga major, in intestinis (Rudolphi). Crotophaga major, Crotophaga ani, Januario, Februario, Julio, Novembri, in intestino coeco (Diesing).

Status: Subulura reclinata (Rudolphi, 1819) Barreto, 1919.

Spiroptera recticauda Molin, 1860

Spiroptera recticauda, Molin, 1860e: 346.

Spiroptera recticauda, Stossich, 1898: 119.

Material: Falco coronatus (Harpyhaliaetus coronatus), 3 우오, Rio Araguay, 31-x-1823, in ventriculo.

Remarks: Molin had only females.

Status: The species is known only from the original description. Until the original material is reviewed, it should be considered species inquirenda.

Dispharagus rectus Molin, 1860

Dispharagus rectus, Molin, 1860b: 494.

Dispharagus rectus, Drasche, 1883b: 209, Taf. XIV, fig. 22.

Acuaria (Cheilospirura) recta, Railliet et al., 1912: 623.

Dispharagus (Synhimanthus) rectus, Gendre, 1921: 39.

Material: Falco unicinctus (Parabuteo unicinctus), 1 , Matogrosso, 29-vi-1827; Falco femoralis, 2 ô $\hat{0}, 1$ q, Caiçara, 2-vi-1826, in eorum ventriculo.

Status: Synhimanthus (Synhimanthus) rectus (Molin, 1860) Gendre, 1921.

Ascaris retusa Rudolphi, 1819

Ascaris retusa, Rudolphi, 1819: 656.

Ascaris retusa, Diesing, 1851: 146.

Schneideria retusa, Travassos, 1926: 88 (pre. oc.).

Schneidernema retusa, Travassos, 1927: 217.

Material: In Dasypus novemcinctus, tuberculis intestinorum crassum (Rudolphi). Dasypus peba (Dasypus novemcinctus), in intestini crassi (Diesing).

Status: Schneidernema retusa (Rudolphi, 1819) Travassos, 1927. 


\section{Physaloptera retusa Rudolphi, 1819}

Physaloptera retusa, Rudolphi, 1819: 258.

Spiroptera retusa, Dujardin, 1845: 104.

Physaloptera retusa, Diesing, 1851: 236.

Physaloptera retusa, Molin, 1860d: 652.

Physaloptera retusa, Ortlepp, 1922: 1027.

Material: (Tupinambis teguixin) in intestinis. (Tupinambis teguixin), $48 \widehat{\jmath}, 93$ + , , Cuyaba, 1-iii-1824 and

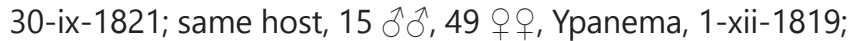
Podinema scripta (?), $20 \hat{\jmath} \widehat{\partial}, 40$ 우, Matogrosso, 20-vii1827 and 27-x-1826; $16 \hat{\jmath} \widehat{\partial}, 39$ oq, same host and locality, 25-xi-1828; Ctenodon nigropunctatus (Tupinambis teguixin), $53 \widehat{\jmath} \hat{\jmath}, 74$ o 9, Ypanema, 19-i-1820 and 26-x-1821; same host, 1 Ĵ, 3 o o, Ytararé, 7-viii-1820; Cnemidophorus lateris-

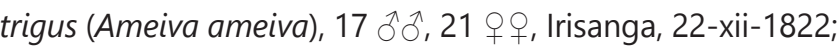
Scleroporus undulatus (Anisolepis undulatus), 1 $\hat{\sigma}, 1$, Cuyaba, 10-iii-1824; $1 \hat{\jmath}$, same host and locality, 8-iii-1824; Amphisbaena flavescens (Amphisbaena alba), $2 \hat{\jmath} \hat{\jmath}$, Ypanema, 26-ii-1819; 1 त, same host and locality, 12-v-1822; Euprepis spixii, 1 ก, 4 q , Cuyaba, 22-iv-1824; Pygodactylus gronovii (Ophiodes striatus), 1 ô, 2 o o, Ypanema, 17-ii-1819; Ophiodes striatus, 2 qo , in eorum ventriculo (Molin). Podinema teguixin (Tupinambis teguexin), Martio, Septembri and Octobri; Podinema graphica (Ameiva ameiva ?), July and November; Tropidurus torquatus (Diesing).

Remarks: Perhaps more than one species is among the exemplars collected by Natterer.

Status: Physaloptera retusa Rudolphi, 1819.

\section{Cheiracanthus robustus Diesing, 1838}

Gnathostoma spinigerum, Owen, 1836: 125.

Cheiracanthus robustus, Diesing, 1838: 189.

Cheiracanthus robustus, Diesing, 1839: 222, Taf. XIV,

fig. 1-7; Taf. XVI, fig. 1-24.

Cheiracanthus robustus, Diesing, 1851: 249.

Cheiracanthus robustus, Drasche, 1882a: 125, Taf. IX,

fig. 3-7.

Material: Felis concolor (Puma concolor), Registo do Jauru, Junio 1826; Forte do Rio Branco, May 1832.

Status: Gnathostoma spinigerum Owen, 1836.

\section{Physaloptera saginata Rudolphi, 1819}

Physaloptera saginata, Rudolphi, 1819: 647.

Spiroptera saginata, Dujardin, 1845: 96.

Physaloptera saginata, Diesing, 1851: 236.

Spiroptera saginata, Molin, 1860e: 347.

Physaloptera saginata, Ortlepp, 1922: 1103.

Spiroptera saginata, Cram, 1926: 207.

Physaloptera saginata, Yamaguti, 1961: 263.

Physaloptera saginata, Hartwich, 1970: 193.
Physaloptera saginata, Vicente et al., 1996: 78.

Material: Strix intestinis Natterer in Brasilia (Rudolphi). Strix atricapilla (Megascops atricapillus), 5 exemplars, Matogrosso, 13-xi-1826, intestino; Strix torquata (Pulsatrix perspicillata), 6 q, , no more data; Falco furcatus (Elanoides forficatus), 2 exemplars, Pará, 30-xi-1830, budello; Crotophaga ani, 1 exemplar, Villa do Manaos, 16-vii-1934; 1 exemplar, same host, Matogrosso, 19-i-1829, and 5 exemplars, same host and locality, 26-ii-1829; Caprimulgus gujanensis (Nyctidromus albicolis), 2 우, Villa do Manaos, 23-vi1834; Caprimulgus leucopygius (Nyctiprogne leucopyga), 1 +, Matogrosso, 4-ix-1826; Icterus cristatus (Psarocolius decumanus), 1 , San Vicente, 20-xii-1826; Thamnophilus funebris (Percnostola rufifrons), 3 오, Barra do Rio Negro, 12 x-1830; Cuculus tinguacu (Piaya cayana), 2 q, , Barra do Rio Negro, 2-v-1833; 1 q, Matogrosso, 30-vii-1827, stomaco; Corvus cajanus (Cyanocorax cayanus), Septembri, and 1 , Barra do Rio Negro, 20-x-1830, 5 q , same locality, 24-ix1830 , in eorum intestinis (Molin).

Remarks: Species inquirendae in Diesing (1851). The species was originally described as a Physaloptera by Rudolphi; later Dujardin (1845) reviewed the material of Rudolphi, passed it to Spiroptera, and gave a size of 34-45 mm. Posteriorly, Molin (1860e) indicated that there is a Spiroptera, not Physaloptera, assigned a size of $30-80 \mathrm{~mm}$. Ortlepp (1922) studied the material of Molin and confirmed that it is not Physaloptera. Cram (1927) placed it as Spiroptera but doubts the size given by Dujardin and Molin; a spirurid of this size in the intestine of birds is doubtful. This viewpoint has led subsequent authors, such as Yamaguti (1961) and Vicente et al. (1996), possibly based on the size, to consider it as Physaloptera, despite the work of Ortlepp (op. cit.).

Status: Until the original material is reviewed, it should be considered incertae sedis.

Aspidocephalus scoleciformis Diesing, 1851

Aspidocephalus scoleciformis, Diesing, 1851: 208.

Aspidocephalus scoleciformis, Diesing, 1855: 180.

Aspidocephalus scoleciformis, Diesing, 1861: 671.

Aspidodera scoleciformis, Railliet \& Henry, 1912: 257.

Material: Dasypus unicinctus (Cabassous tatovay), Dasypus gilvipes (Euphractus sexcinctus), Octobri and Augusto; Dasypus tricinctus (Tylopeutes tricinctus), Novembri; Didelphis murina (Marmosa murina), Didelphis domestica (Monodelphis domestica), Novembri, in intestinis.

Remarks: Probably there is more than one species, depending on the host.

Status: Aspidodera scoleciformis (Diesing,1851) Railliet \& Henry, 1912. 
Monodontus semicircularis Molin, 1861

Monodontus semicircularis, Molin, 1861a: 469.

Material: Dicotyles torquatus (Pecari tajacu), $17 \hat{\jmath} \sigma^{2}, 27$

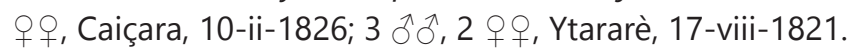

Status: Monodontus semicircularis Molin, 1861.

Physaloptera semilanceolata Molin, 1860

Physaloptera semilanceolata, Molin, 1860d: 659.

Physaloptera semilanceolata, Drasche, 1882a: 127, Taf.

VIII, fig. 15 und 16.

Physaloptera semilanceolata, Ortlepp, 1922: 1017.

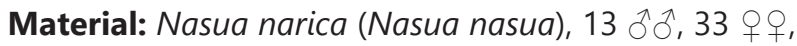

Nas Lages, 3-iv-1833, in ventriculo.

Status: Physaloptera semilanceolata Molin, 1860.

\section{Spiroptera semilunaris Molin, 1860}

Spiroptera semilunaris, Molin, 1860a: 936.

Spiroptera semilunaris, Drasche, 1883b: 197, Taf. XI, fig.

23; Taf. XIV, fig. 10.

Cyrnea semilunaris, Seurat, 1914d: 153.

Material: Trogon collaris, $2 \hat{\delta} \bar{\delta}, 2$ q, , Villa Maria, 28viii-1825; Trogon melanurus, 3 우, Barra do Rio Negro, 16-vii-1833; 14 우, Villa Maria, 20-ix-1825. Inter tunicas ventriculi.

Status: Cyrnea semilunaris (Molin, 1860) Seurat, 1914.

\section{Filaria serotina Molin, 1858}

Filaria serotina, Molin, 1858b: 374.

Material: Lichenops perspicillata (Hymenops perspicillatus), 1 , Pausecco, Junio, in cavo abdominis.

Status: The species has been overlooked by authors. Until review of the sample, it must be incertae sedis.

\section{Ascaris serpentulus Rudolphi, 1809}

Ascaris ardeae, Frölich, 1802: 44.

Ascaris serpentulus, Rudolphi, 1809: 191.

Ascaris serpentulus, Rudolphi, 1819: 663.

Ascaris serpentulus, Diesing, 1851: 156.

Porrocaecum serpentulus, Baylis, 1920: 261.

Porrocaecum serpentulus, Hartwich, 1964b: 64.

Material: Ardea nycticorax (Nycticorax nycticorax), in intestinis (Rudolphi). Ardea nycticorax (Nycticorax nycticorax), Februario, in intestinis; Ardea pileata (Philerodius pileatus), Julio, in oesophagus; Ardea caerulea (Egretta caerulea), Aprili, in intestinis; Ardea agami (Agamia agami), Octobri, in diverticulum (Diesing).

Status: Porrocaecum serpentulus (Rudolphi, 1809) Baylis, 1920.

Spiroptera serpentulus Diesing, 1851

Spiroptera falconis, Rudolphi, 1819: 254.
Spiroptera serpentulus, Diesing, 1851: 221.

Spiroptera serpentulus, Diesing, 1857: 14.

Spiroptera serpentulus, Molin, 1860a: 961.

Spiroptera serpentulus, Drasche, 1883b: 202, Taf. XIII,

fig. 18 und 19.

Pelecitus falconis, Baylis, 1944: 800.

Material: Falco cachinans (Herpetotheres cachinnans), 1 , Caiçara, 24-x-1825, in articulationibus calcium; Falco aquilinus (Ibycter americanus), 15 9 , Mattogrosso, 10-x1826, in articulatione calcis; Falco gracilis (Geranospiza caerulescens), $1 \hat{\jmath}, 1$, Rio Araguay, in articulatione calcis; same host, 4 우, Cuyaba, 2-xi-1823 and 21-i-1825; Falco milvoides (Busarellus nigricollis), 1 o, 1 +, Rio Araguay, 28$\mathrm{x}$-1823; Falco magnirostris (Rupornis magnirostris), 1 ô, 3 9 , Caiçara, 11-i-1826, inter tendines articulationis calcis; Falco urubutinga (Buteogallus urubutinga), $9 \hat{\jmath}, 23$ q, Caiçara, 19-iv-1826, in articulatione calcis et inter tendines digitorum pedum; Falco melanops (Leucopternis melanops), 52 exemplars, Pará, 1-i-1835; Falco unicinctus (Parabuteo unicinctus), 5 ふึ, 11 우, Mattogrosso, 16-xii-1828, inter tendines ad articulationem ulnae; Falco nitidus (Buteo nitidus), 2 ふैग, 2 우, Rio Araguay, 31-x-1823, libere inter tendines cubiti alae; Falco destructor (Harpia harpyja), 1 q, Barra do Rio Negro, 15-v-1833, inter tendines digiti pedis; Picus lineatus (Dryocopus lineatus), 2 q, Irisanga, 10-iii1823, in articulatione calcis, Martio; Picus robustus (Phloeoceastes robustus), $5 \hat{\jmath} \hat{\jmath}, 7$ q, Caiçara, 24-x-1825, in articulatione calcis.

Remarks: Diesing (1851) without justification renamed falconis to serpentulus.

Status: Pelecitus falconis (Rudolphi, 1819) Baylis, 1944.

Filaria serpicula Molin, 1858

Filaria serpicula, Molin, 1858b: 385.

Solenonema serpicula, Diesing, 1861: 705.

Solenonema serpiculum, Yorke \& Maplestone, 1926: 439.

Litomosoides serpicula, Guerrero et al., 2002: 184.

Material: Phyllostoma brevicaudatum (Carollia brevi-

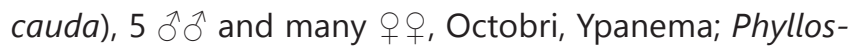
toma spiculatum (nomen nudum), Januario, Ypanema; Phyllostoma sp., Februario, Rio Muria. In eorum cavo abdominis.

Remarks: The only collected bat in Ypanema in January is Sturnira lilium.

Status: Litomosoides serpicula (Molin,1858) Guerrero et al., 2002.

Ascaris serrata Drasche, 1883

Ascaris serrata, Drasche, 1883a: 116, Taf. V, fig. 3 und 4. Ascaris draschei, Stossich, 1896: 24.

Porrocaecum draschei, Baylis, 1927: 36. 
Terranova serrata, Mosgovoi, 1951: 542.

Terranova (s.l.) serrata, Moravec \& Justine, 2020: 11.

Material: Vastres cuvieri (Arapaima gigas), $1 \hat{\sigma}$, no more data.

Remarks: Drasche (1883a) pointed out "in a bottle labeled Vastres Cuvieri containing exemplars of Lecanocephalus was a male of Ascaris." The sample would be the same Lecanocephalus spinulosus (Drasche, I.c., p. 107) labeled "Lecanocephalus denticulatus D. Sudis gigantis" used by Diesing to describe $L$. spinulosus. Unfortunately, the type material of this species is Sudis gigas from Borba, iv-1830, and Forte do Rio Branco, iv-1832 (Diesing, 1839: 227), so the type locality of $C$. tridentatus is uncertain. Stossich (1896) renamed this species as Ascaris draschei, not Ascaris serrata Wright, 1879, and later as a Spirurid, so the renamed $A$. serrata of Drasche was unnecessary.

Status: Species inquirenda in agreement with Moravec \& Justine (2020).

\section{Spiroptera sexalata Molin, 1860}

Spiroptera strongylina, Rudolphi, 1819: 237.

Spiroptera strongylina, Diesing, 1851: 211 (in partim).

Spiroptera sexalata, Molin, 1860a: 957.

Physocephalus sexalatus, Diesing, 1861: 686.

Physocephalus sexalatus, Drasche, 1883b: 213, Taf. XII,

fig. 25; Taf. XIV, fig. 3 und 4.

Spiroptera sexalata, Stossich, 1898: 105.

Material: Dicotyles albirostris (Tayassu pecari), $6 \hat{\jmath} \widehat{\jmath}, 77$ qo, Caiçara, 24-iv-1826, in ventriculo.

Status: Physocephalus sexalatus (Molin, 1860) Diesing, 1861.

Filaria simplicissima Molin, 1858

Filaria simplicissima, Molin, 1858b: 372.

Filaria simplicissima, Vicente et al., 1996: 102.

Material: Psittacus makaonanna (Orthopsittaca manilata), 2 q, Egenho do Cap Gama, Julio, sub cute ad aurem dextrum.

Status: Molin (1858b) found only females. Until review of the material, it must remain as incertae sedis. Same in Vicente et al. (1996).

\section{Spiroptera singularis Molin, 1860}

Spiroptera singularis, Molin, 1860a: 941.

Spiroptera singularis, Drasche, 1883b: 199, Taf. XI, fig. 12-14.

Cyrnea singularis, Chabaud, 1958: 490.

Material: Cathartes urubu (Cathartes burrobianus), 8 qo, Ypanema, 31-xii-1821, inter tunicas ventriculi.

Remarks: Molin (1860a) described the species based on females, and it has not been found subsequently. According to figures of Drasche (1883b) and Chabaud (1958), it was included within the genus Cyrnea as "insufficiently known," but according to the position of the vulva it is Procyrnea.

Status: Procyrnea singularis (Molin, 1860) n. comb.

\section{Filaria sphaerophora Molin, 1858}

Filaria sphaerophora, Molin, 1858b: 401, Taf. II, fig. 8.

Material: Anabates (Anumbis) anthoides (Anumbius annumbi), 4 우, Ytararé, Augusto 1831; Fazenda do Rio Verde, Augusto 1820; Muscicapa lophotes (Knipolegus lophotes), in hepate.

Remarks: As described by Molin (1858b) and his figures, the species is very peculiar but has been overlooked by the authors.

Status: Until the material is reviewed, it should be considered as incertae sedis.

\section{Ascaris spiculigera Rudolphi, 1809}

Ascaris spiculigera, Rudolphi, 1809: 168.

Ascaris variegata, Rudolphi, 1809: 169.

Ascaris spiculigera, Rudolphi, 1819: 662.

Ascaris spiculigera, Diesing, 1851: 157.

Contracaecum spiculigera, Hartwich, 1964a: 26.

Material: Pelecanus aquila (Fregata aquila) (Rudolphi). Podiceps dominicensis (Tachybaptus dominicus), Carbo brasiliensis (Tigrisoma lineatum), Octobri; Tachypetes aquila (Fregata magnificens), Martio (Diesing). Oesophagus et ventriculo.

Status: Synonym of Contracaecum microcephalum (Rudolphi, 1809) in Hartwich (1964c).

Ascaris spinicauda Olfers, 1819

Ascaris spinicauda, Olfers in Rudolphi, 1819: 272.

Ascaris spinicauda, Rudolphi, 1819: 652.

Ascaris spinicauda, Diesing, 1851: 150.

Spinicauda spinicauda, Travassos, 1920a: 43.

Material: Scinci sp. n. 9. (Euprepidis spixii), Gymnodactylus geckoides (?), in palpebris (Rudolphi). Podinema teguixin (Tupinambis teguixin), Septembri et Octobri; Ctenodon nigropunctatus (Tupinambis teguixin), intestino crasso (Diesing).

Status: Spinicauda spinicauda (Olfers in Rudolphi, 1819) Travassos, 1920.

Lecanocephalus spinulosus Diesing, 1839

Lecanocephalus spinulosus, Diesing, 1839: 227, Taf. XIV, fig. 12-20.

Lecanocephalus spinulosus, Diesing, 1851: 250. Lecanocephalus spinulosus, Drassche, 1883a: 107, Taf. III, fig. 10-16.

Goezia spinulosa, Railliet \& Henry, 1915a: 274. 
Material: Sudis gigas (Arapaima gigas), Borba, Januario et Aprili, 1830; Forte do Rio Branco, April 1832, im magen (stomach).

Status: Goezia spinulosa (Diesing, 1839) Railliet \& Henry, 1915.

\section{Ascaris spiralis Molin, 1860}

Ascaris spiralis, Molin, 1860e: 339.

Ascaris spiralis, Diesing, 1861: 667.

Dispharynx nasuta, Goble \& Kutz, 1945: 329.

Material: Picus comatus (Campephilus melanoleucus), 6 exemplars, Barra do Rio de Janeiro, 8-x-1825.

Status: Synonym of Dispharynx nasuta (Rudolphi, 1819) in Goble \& Kutz, 1945.

\section{Spiroptera spiralis Molin, 1860}

Spiroptera spiralis, Molin, 1860a: 947.

Filaria spiralis, von Linstow, 1879: 326, Tav. V, fig. 10. 23.

Spiroptera spiralis, Drasche, 1883b: 200, Taf. XIV, fig.

Oxyspirura spiralis, Stossich, 1898: 125.

? Onchocerca spiralis, Yorke \& Maplestone, 1926: 414.

Dipetalonema spiralis, Sandground, 1938: 423.

Dipetalonema spiralis, Lent \& Freitas, 1942: 383.

Deraiophoronema spiralis, Yeh, 1957: 199.

Bostrichodera spiralis, Chabaud \& Bain, 1976: 368.

Material: Bradypus didactylus (Choloepus didactylus), 1 j, 13 우, Rio Hié, 31-v-1831; same host, 3 우, Marabitanas, 22-i-1831, sub tendinibus digitorum pedum posteriorum.

Status: Bostrichodera spiralis (Molin, 1860) Chabaud \& Bain, 1976.

Filaria striata Molin, 1858

Filaria striata, Molin, 1858b: 388.

Solenonema striatum, Diesing, 1861: 705.

Dirofilaria striata (Molin, 1858), Railliet \& Henry,

1911b: 487.

Dirofilaria striata, Vicente et al., 1998: 170.

Material: Felis concolor (Puma concolor), many $\hat{\sigma} \hat{\partial}$ and 우, Caiçara, 18-xi-1825, sub cute abdominis inter fibras musculares; Felis macrura (Leopardus wiedii), Quartel do Poste, 14-vi-1819, inter cutem et musculus antibrachii.

Remarks: Yorke and Maplestone (1926) included Dirofilaria striata (p. 394) and Solenonema striata (p. 439) as different species; the same mistake is committed by Yamaguti (1961). However, López Neyra (1956) and Sonin (1975) corrected the error.

Status: Dirofilaria striata (Molin, 1858) Railliet \& Henry, 1911.
Filaria strigis torquatae Molin, 1858

Filaria strigis torquatae, Molin, 1858b: 422.

Filaria attenuatta, Stossich, 1898: 55.

Material: Strix torquatua (Pulsatrix perspicillata), Octobri, in abdomine.

Remarks: Molin (1858b) had a single opaque exemplar from which he could not determine the sex and assigned it to Filaria attenuata. He believed the material from Strix (s.l.) named by Diesing as Filaria attenuata is a different species; in fact, under the name of Filaria attenuata there are several species, so Molin (loc. cit.) concluded it is species inquirendae.

Status: In any case, the species should be regarded as nomen nudum.

Ascaris strongylina Rudolphi, 1819

Ascaris strongylina, Rudolphi, 1819: 651.

Ascaris strongylina, Diesing, 1851: 150.

Subulura strongylina, Railliet \& Henry, 1912: 258.

Subulura strongylina, Hartwich, 1965: 127.

Material: Crypturus (Tinamus n. 171) (Crypturus tautapa) and Tetraonis uru (n. 173) (Odontophorus capieira), in intestinis (Rudolphi). Perdix dentata (Odontophorus capieira), Tinamus tutaupa (Crypturus tautapa), caecum (Diesing).

Status: Subulura strongylina (Rudolphi, 1819) Railliet \& Henry, 1912.

Physaloptera strongylina Rudolphi, 1819

Physaloptera strongylina, Rudolphi, 1819: 646.

Spiroptera affinis, Dujardin, 1845: 98.

Physaloptera strongylina, Diesing, 1851: 235.

Physaloptera strongylina, Stossich, 1889: 53.

Physaloptera strongylina, Ortlepp, 1922: 1106.

Physaloptera strongylina, Hartwich, 1970: 193.

Material: Cuculus seniculus (Coccyzus minor), in ventriculo et intestinis.

Remarks: Species inquirenda in Sossich (1889). Both Molin (1860) and Ortlepp (1922) ruled this species out of Physaloptera.

Status: Incertae sedis.

Spiroptera strongylina Rudolphi, 1819

Spiroptera strongylina, Rudolphi, 1819: 237.

Spiroptera strongylina, Diesing, 1851: 211.

Spiroptera strongylina, Stossich, 1898: 92.

Ascarops strongylina, Alicata \& McIntosh, 1933: 62.

Material: Dicotyles albirostris (Tayassu pecari), Registro do Say, 18-iv-1818, in ventriculo.

Status: Ascarops strongylina (Rudolphi, 1819) Alicata \& McIntosh, 1933. 
Diaphanocephalus strongyloides Diesing, 1851

Diaphanocephalus strongyloides, Diesing, 1851: 297.

Diaphanocephalus strongyloides, Diesing, 1855: 182.

Diaphanocephalus strongyloides, Diesing, 1861: 715.

Diaphanocephalus strongyloides, Molin, 1861a: 581.

Diaphanocephalus galeatus, Railliet \& Henry, 1909b: 171.

Material: Podinema teguixin (Tupinambis teguixin), Novembri, Brasil, in intestinis (Diesing, 1851). Podinema te-

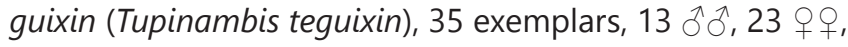
Mattogrosso, 29-x-1826 and 22-xi-1826; same host, 198 ô $\hat{\jmath}, 263$ q + , Ypanema, 12-xi-1821, 24-xi-21, and 30-ix1831; Ctenodon nigropunctatus (Tupinambis nigropunc-

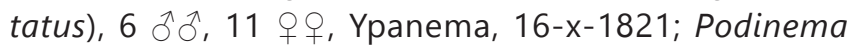
scripta (?), 6 ô, 11 우, Mattogrosso, 1-xi-1826; Thorichtis Dracaena (Dracaena guianensis), $103 \hat{\jmath} \widehat{\partial}, 113$ 우, Mattogrosso, 20-v-1828. In intestini tenue.

Status: Synonym of Diaphanocephalus galeatus (Rudolphi, 1819) Railliet \& Henry, 1909.

\section{Kalicephalus strumosus Molin, 1861}

Kalicephalus strumosus, Molin, 1861a: 542.

Kalicephalus costatus costatus, Schad, 1962: 1051.

Material: Coluber Lichtensteinii (Mastigodryas bifossatus), $4 \hat{\partial} \hat{\partial}, 7$ q $q$, Mattogrosso, 13-xii-1828, 27-i-1829, and 4-ii-1829. In intestino.

Status: Synomym of Kalicephalus costatus costatus (Rudolphi, 1819) Schad, 1962.

\section{Spiroptera subaequalis Molin, 1860}

Spiroptera subaequalis, Molin, 1860a: 921.

Spiroptera subaequalis, Drasche, 1883b: 194, Taf. XI,

fig. 5; Taf. XIII, fig. 9.

Cylicospirura subaequalis, Vevers, 1922: 910.

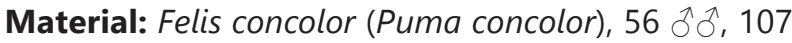
q+ , Forte do Rio Branco, 9-v-1832, in intestino tenui; 11

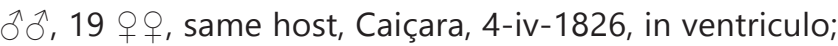
Felis mellivora (Herpailurus yagouaroundi), $29 \hat{\partial} \partial^{\lambda}, 17$ 우, Caiçara, 21-iv-1826, in ventriculo.

Status: Cylicospirura subaequalis (Molin, 1860) Vevers, 1922.

Strongylus subauricularis Rudolphi, 1819

Strongylus subauricularis, Rudolphi, 1819: 649.

Strongylus subauricularis, Diesing, 1851: 315.

Oswaldocruzia subauricularis, Travassos, 1917a: 73.

Material: Rana musica (Rhinella icterica), in intestinis (Rudolphi). Docidophryne agua (Rhinella icterica) (Diesing).

Remarks: See Strongylus auricularis.

Status: Oswaldocruzia subauricularis (Rudolphi, 1819) Travassos, 1917.
Filaria subspiralis Diesing, 1851

Filaria subspiralis, Diesing, 1851: 268.

Filaria subspiralis, Molin, 1858b: 391.

Lemdana subspiralis (Diesing, 1851), López-Neyra, 1956: 188.

Material: Ardea leucogaster (Egretta tricolor), several $\widehat{\partial} \widehat{\partial}$ and $q$ 우, Praja de Cajutuba, inter tendonis digitorum.

Status: Lemdana subspiralis (Diesing, 1851) LopezNeyra, 1956.

Trichocephalus subspiralis Diesing, 1851

Trichocephalus subspiralis, Diesing, 1851: 294.

Trichocephalus subspiralis, Diesing, 1857: 17, Tab. II, fig. 37-44.

Material: Dasypus tricinctus (Tolypeutes matacus), $2 \widehat{\partial} \partial^{\lambda}$, 2 우, Mattogrosso, 26-xi-1826; Dasypus unicinctus (Cabassous tatouay), Ypanema, 16-ii-1819, same host, Caiçara, 13x-1825; Dasypus loricatus (Cabassous tatouay), November, in eorum intestino coeco.

Remarks: The species has not been mentioned since 1857, but it is certainly a Trichuris.

Status: Trichuris subspiralis n. comb.

Ascaris subulata Rudolphi, 1819

Ascaris subulata, Rudolphi, 1819: 269.

Ascaris caprimulgi, Rudolphi, 1819: 55.

Ascaris forcipata, Rudolphi, 1819: 659 (Caprimulgi et

Cuculi).

Ascaris subulata, Diesing, 1851: 173.

Travassallodapa subulata, Lopez-Neyra, 1945: 300.

Ascaris subulata, Inglis, 1958: 601.

Subulura spp., Vicente et al., 1996: 46, 47.

Material: Caprimulgus cortopan (Antrostomus rufus), Engenho do Capitão Gama, Augusto, and locality ?, Octobri; Caprimulgus mercurius (Eleothreptus anomalus), Januario; Caprimulgus candicans (Eleothreptus candicans), Irisanga, Januario; Caprimulgus diurnus (Podager nacunda), Novembri, Aprili et Junio; Caprimulgus nattereri (Lurocalis semitorquatus), Martio, Septembri et Octobri; Caprimulgus trifurcus (Hydropsalis climacocerca), Augusto; Caprimulgus scaphiurus, Octobri; Caprimulgus semitorquatus (Lurocalis semitorquatus); Caprimulgus guianensis (Nyctidromus albicollis); Nyctibius aethereus; Nyctibius grandis, Mattogrosso, Septembri, and Caiçara, Januario; Cuculus cayanus (Piaya cayana), Februario; Cuculus tinguazu (Piaya cayana); Cuculus naevius (Tapera naevia); Cuculus melacoryphus (Coccyzus melacoryphus), in intestino coeco.

Status: Subulura forcipata (Rudolphi, 1819) Railliet \& Henry, 1912 (p.p.); Subulura strongylina (Rudolphi, 1819) Railliet \& Henry, 1912 (p.p.); Subulura suctoria (Molin, 1860) Railliet \& Henry, 1912 (р.p.). 
Oesophagostomum subulatum Molin, 1861

Strongylus dentatus, Rudolphi, 1803: 12.

Sclerostomum dentatum, Diesing, 1851: 305.

Oesophagostomum subulatum, Molin 1861a: 445.

Oesophagostomum dentatus, Railliet, 1895: 451.

Material: Sus scrofa, $8 \hat{\jmath} \hat{\jmath}, 14+q$, Cuyaba, 8-vii-1824;

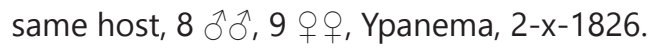

Status: Synonym of Oesophagostomum dentatum (Rudolphi, 1803) in Railliet (1895).

\section{Histiocephalus subulatus Molin, 1860}

Histiocephalus subulatus, Molin, 1860c: 513.

Histiocephalus subulatus, Drasche, 1883b: 208, Taf. XII, fig. 5-7; Taf. XIV, fig. 12.

Aspidodera subulata, Railliet \& Henry, 1912: 257.

Material: Didelphis myosurus (Metachirus nudicaudatus), 1 đ., Ypanema, 25-i-1822, in ventriculo.

Remarks: Species inquirenda in agreement with Molin (1860c) because he had only one male and appeared to belong to this genus; however, it was in perfect condition and was described and figured by Drasche (1883b), who reassigned it to Aspidocephalus, a synonym of Aspidodera.

Status: Aspidodera subulata (Molin,1860) Railliet \& Henry, 1912.

\section{Kalicephalus subulatus Molin, 1861}

Kalicephalus subulatus, Molin,1861a: 543.

Material: Lachesis rhombeata (Lachesis muta), $15 \hat{\jmath}$, 36 우, Borba, vii-1830; $12 \hat{\jmath} \hat{\jmath}, 16$ $q$, same host and locality, 28-vi-1830; Bothrops jararaca, 1 $\hat{\text {, }} 1$ q, Ypanema, 22-vii-

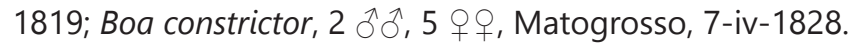

Status: Kalicephalus subulatus Molin, 1861.

\section{Heteracis suctoria Molin, 1860}

Heteracis suctoria, Molin, 1860e: 341.

Heteracis suctoria, Diesing, 1861: 643.

Heteracis suctoria, Drasche, 1882a: 119, Taf. VII, fig.

5-10.

Subulura suctoria, Railliet \& Henry, 1912: 257.

Material: Caprimulgus campestris (Podager nacunda), 1 ふ., Manaos, 19-vi-1834, inter tunicas ventriculi.

Status: Subulura suctoria (Molin, 1860) Railliet \& Henry, 1912.

\section{Dispharagus sygmoideus Molin, 1860}

Dispharagus sygmoideus, Molin, 1860b: 483.

Dispharagus sygmoideus, Stossich, 1891: 100.

Acuaria sygmoidea, Railliet et al., 1912: 624.

Synhimantus sigmoideus, Cram, 1927: 282.

Material: Falco tridentatus (Leucopernis sp.), $1 \hat{\sigma}$, in Brasilia (no more data).
Remarks: Species inquirenda in Stossich (1891) but not in Molin (1860b).

Status: Synhimantus (Synhimantus) sygmoideus (Molin, 1860) Cram 1927.

Filaria tantali cayennensis Molin, 1858

Filaria tantali cayennensis, Molin, 1858b: 435.

Material: Ibis cayennensis (Mesembrinis cayennensis), Aprili, Cuyaba, inter tunicas proventriculi.

Remarks: There is not a description. In agreement with Molin (1858b) that it must be Strongyloidea.

Status: It is a nomen nudum.

Ascaris tentaculata Rudolphi, 1819

Ascaris tentaculata, Rudolphi, 1819: 658.

Ascaris tentaculata, Diesing, 1851: 147.

Cruzia tentaculata, Travassos, 1917b: 100.

Cruzia tentaculata, Hartwich, 1964c: 256.

Material: Didelphis ocuaiquiqua (Nomen nudum) (forsan ejusdem speciei ?), in illo (Rudolphi). Didelphis philander (Caluromys philander), Januario, Septembri, Martio, Majo; Didelphis quica (Philander opossum); Didelphis domestica (Monodelphis domestica), Martio; Didelphis opossum (Didelphis marsupialis), Decembri; Didelphis virginiana (Didelphis albiventris), Julio; Didelphis cancrivora (Didelphis marsupialis); Didelphis lanigera (Caluromys lanatus), Novembri; in intestinis coecis (Diesing).

Status: Cruzia tentaculata (Rudolphi, 1819) Travassos, 1917.

Spiroptera tenuicauda Molin, 1860

Spiroptera tenuicauda, Molin, 1860a: 983.

Spiroptera tenuicauda, Drasche, 1883b: 207, Taf. XII, fig. 17.

Material: Ciconia maguari, $2 q q$, Forte do Rio Branco, 22-iv-1831, in proventriculo; Crax tomentosa (Pauxi tomentosa), 2 우, Marabitanas, 22-i-1831, sub membrana nictitante.

Remarks: Species inquirenda in agreement with Molin (1860a), who examined 2 females from each host. Molin doubted that both groups are of the same species but having no male could not decide. No doubt both species should be different. Drasche gives an apical view with a hexagonal mouth opening and two cephalic alae but does not specify from which host it comes. If Pauxi, it could be a kind of Oxyspirura (s.l.); also, in his description, the oesophagus includes a terminal bulb and could be a female and an oxyurid with the material from Ciconia.

Status: Until review of the material, it should keep the name incertae sedis. 
Ascaris tenuicolis Rudolphi, 1819

Ascaris tenuicolis, Rudolphi, 1819: 286.

Ascaris tenuicolis, Diesing, 1851: 160.

Multicaecum tenuicolis, Hartwich, 1964b: 65.

Brevimulticaecum brevicolle, Sprent, 1979a: 109.

Material: Champsa sclerops (Caiman crocodilus), Augusto et Novembri; Champsa nigra (Melanosuchus niger), Septembri.

Status: Brevimulticaecum tenuicolle (Rudolphi, 1819) Sprent, 1979.

\section{Spiroptera tercostata Molin}

Spiroptera tercostata, Molin, 1860a: 947.

Pelecitus tercostatus, Railliet \& Henry, 1910b: 251.

Material: Psittacus aestivus (Amazona aestiva), in two hosts, $11 \hat{\jmath} \hat{\jmath}, 27$ oq, Murumgaba, 4-iv-1821, and Rio das Flechas, 21-vii-1825, in junctura calcis; Psittacus Maximiliani (Pionus maximiliani), 1 o, 5 우, Ypanema, 25-ii-1825, inter musculos juncturae calcis.

Status: Pelecitus tercostatus (Molin, 1860) Railliet \& Henry, 1910.

\section{Physaloptera terdentata Molin, 1860}

Physaloptera terdentata, Molin, 1860d: 651.

Physaloptera terdentata, Drasche, 1882a: 127, Taf. VIII,

fig. 17 und 18.

Physaloptera terdentata, Ortlepp, 1922: 1048.

Material: Felis concolor (Puma concolor), $1 \hat{\delta}$, Forte do Rio Branco; Felis tigrina (Leopardus tigrinus), 2 + $q$, in oorum ventriculo.

Remarks: Ortlepp pointed out "three bottles of material, Y 1074 from Felis concolor, 4511 from Felis tigrina, and 4513 from Felis sp. labelled Ph. dentata and deposited in the Vienna Museum, were examined, . . . bottles 4511 and 4513 proved to be not $P$ h. terdentata but $P$. praeputialis ... the material from Felis concolor proved to be Ph. terdentata."

Status: Physaloptera terdentata Molin, 1860.

\section{Spiroptera terdentata Molin, 1860}

Spiroptera terdentata, Molin, 1860a: 944.

Spiroptera terdentata, Drasche, 1883b: 199, Taf. XI, fig.

25; Taf. XIII, fig. 24.

Spiroptera terdentata, Stossich, 1898: 102.

Material: Tinamus variegatus (Crypturellus variegatus), $2 \widehat{\jmath}$, Borba, 5-vii-1830, inter tunicas ventriculi.

Remarks: I agree with the description and figures of Drasche (1883b) that it must be Procyrnea.

Status: Procyrnea terdentata (Molin, 1860) n. comb.
Cucullanus tridentatus Drasche, 1883

Cucullanus tridentatus, Drasche, 1883a: 116, Taf. V, fig. 1 und 2.

Camallanus tridentatus, Railliet \& Henry, 1915b: 449.

Material: Vastres cuvieri (Arapaima gigas), 1 q.

Remarks: Drasche (1883a) pointed out "in a bottle labeled Vastres Cuvieri containing exemplars of Lecanocephalus was a female of Cucullanus." The sample would be same Lecanocephalus spinulosus (Drasche, I.c., p. 107) labeled "Lecanocephalus denticulatus D. Sudis gigantis" used by Diesing to describe $L$. spinulosus. Unfortunately the type material of this species is: Sudis gigas from Borba, iv-1830, and Forte do Rio Branco, iv-1832 (Diesing, 1839: 227), so the type locality of $C$. tridentatus is uncertain.

Status: Camallanus tridentatus (Brasche,1883) Railliet \& Henry, 1915.

Filaria terebra Diesing, 1851

Filaria cervi, Rudolphi, 1819: 8.

Filaria cervina, Dujardin, 1845: 49.

Filaria terebra, Diesing, 1851: 274.

Artionema bidentata, Rego, 1960: 149.

Setaria bidentata, Vicente et al., 1998: 175.

Material: Cervus nambi (Mazama gouazoubira), Caiçara, 12-iii-1826, Villa Maria, 25-ix-1825; Cervus simplicicornis (Mazama gouazoubira), Cervus rufus (Mazama rufa), Junio und Decembri, in eorum cavo abdominali.

Remarks: Diesing based his species on material from Cervus elaphus, a palaearctic species, and apparently the same sample of Rudolphi (1819) and Dujardin (1845), but he also included material collected by Natterer from Brazil. Later, Molin (1858b) considered only the material from Cervus elaphus, and samples from Brazil are in Filaria bidentata. Rego (1960) synonymized it with Artionema bidentata.

Status: Synonymous with Setaria bidentata in Vicente et al. (1998).

Cyathostomum tetracanthum (Mehlis, 1831)

Strongyli armati proles, Rudolphi, 1808: 323.

Strongylus tetracanthus, Mehlis, 1831: 79.

Sclerostoma quadridentatum, Dujardin, 1845: 258.

Sclerostomum tetracanthum, Diesing, 1851: 305.

Sclerostoma hexacanthum, Wedl, 1856: 53, pl. 3, fig. 31-33.

Cyathostomum tetracanthum, Molin, 1861a: 453.

Material: Equus mulus, Januario, in intestino coeco (Diesing). Equus mulus, many $\widehat{\partial} \widehat{\partial}$ and $q+{ }_{+}$, Irisanga, 4-i1823 (Molin).

Status: Cyathostomum tetracanthum (Mehlis, 1831). 
Spiroptera thamnophili Molin, 1860

Spiroptera thamnophili, Molin, 1860a: 980.

Material: Thamnophilus tigrinus (Mackenziaena leachi),

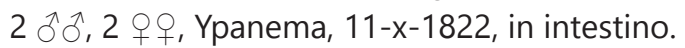

Remarks: Species inquirenda in agreement with Molin; the exemplars were in poor condition. No mention of this species is in Drasche's papers-perhaps the material was lost.

Status: Until the original material is reviewed, it should be considered incertae sedis or nomen nudum.

\section{Filaria tinami variegati Molin, 1858}

Filaria tinami variegati (palpebralis), Molin, 1858b: 427.

Filaria tinami, Yorke \& Maplestone, 1926: 439.

Tetracheilonema quadrilabiata, Yorke \& Maplestone, 1926: 439.

Tetracheilonema quadrilabiata, Yamaguti, 1961: 315.

Material: Tinamus variegatus (Crypturellus variegatus), 1 exemplar, Barra do Rio Negro, Octobri, sub membrana nictitante.

Remarks: Species inquirenda in agreement with Molin (1858b), who examined a single specimen collected by Natterer in a poor state of preservation, which seemed to be a female belonging to Filaria labiotruncata, but he separated it from the latter on account of the difference in habitat. Yorke and Maplestone (1926) included the species under Tetracheilonema quadrilabiata together with Filaria labiotruncata, same as Yamaguti (1961).

Status: Tetracheilonema is not an eye worm, so the species must be species inquirenda or nomen nudum.

\section{Filaria torta Molin, 1858}

Filaria torta, Molin, 1858b: 390.

Filaria torta, Stossich, 1898: 83.

Filaria torta, Webber, 1955: 239.

Material: Cebus lagothrix (Lagothrix lagotricha ?), no more data.

Remarks: The species has been overlooked by all authors less Webber (1955), who only quotes it. Molin (1858b) had numerous males and females, but after the original description, it cannot be recognized as one of the more than 20 species of known filariae from neotropical monkeys. The described species from Lagothrix are Dipetalonema filiformis and Dipetalonema caudispina, both much larger than Filaria torta, so it must be some sort of Dipetalonema or Mansonella (Tetrapetalonema).

Status: Until review of the original material, it should be considered incertae sedis.
Filaria tricoronata Molin, 1858

Filaria tricoronata, Molin, 1858b: 400.

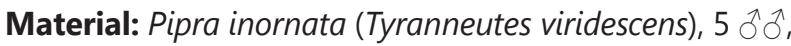
7 우, Borba, Majo, in cavo abdominis.

Status: The species is well characterized by a double circle of cephalic papillae, but until the original material is reviewed, it should be considered incertae sedis.

Filaria tridens Molin, 1858

Filaria tridens, Molin, 1858b: 393.

Diplotriaena tridens, Boulenger, 1928: 49.

Material: Icterus cristatus (Psarocolius decumanus), Barra do Rio Negro, Octobri, inter tunicas parietis abdominis et in cavo abdominis; Icterus haemorrhous (Cacicus haemorrhous), Tybaya, Novembri; Icterus (Psarocolius) icterocephalus (Chrysomus icterocephalus), Rio Amazonas, Septembri; Icterus chopi (Gnorimopsar chopi), Ypanema, Aprili, in eorum cavo abdominis; same host, Ypanema, Augusto, in regione renum; Icterus sericeus (Molothrus bonariensis), Joanna Leite, Novembri, in cavo abdominis et in regione renum; Cassicus ater (Molothrus oryzivorus), Ypanema, Septembri, in cavo and thorac. et abd., nec non plures sub renibus; same host, Pará, Decembri, in cavo abdominis; Cassicus viridis (Psarocolius viridis), Barra do Rio Negro, Septembri, in cavo et inter tunicas parietum abdominis.

Status: Diplotriaena tridens (Molin, 1858) Boulenger, 1928.

Dochmius trigonocephalus Rudolphi, 1809

Strongylus vulpis, Zeder, 1800: 73.

Strongylus trigonocephalus, Rudolphi, 1809: 231.

Strongylus tetragonocephalus, Rudolphi, 1809: 232.

Dochmius trigonocephalus, Dujardin, 1845: 277.

Dochmius trigonocephalus, Diesing, 1851: 300.

Dochmius trigonocephalus, Molin, 1861a: 67.

Bunostomum trigonocephalus, Railliet, 1902: 108.

Material: Canis jubatus (Chrysocyon brachyurus), Octobri; Canis azarae (Cerdocyon thous), Novembri (Diesing). Canis familiaris venaticus (Speothos venaticus), $50 \hat{\partial} \partial^{\lambda}, 64$ 우오, Caiçara, 16-iv-1826; Canis azarae (Cerdocyon thous), 30 के, 86 क , as follows: 110 from Registo do Rio Araguay, 15-xi; 2, Caiçara, 17-xi-1825; 4, same locality, 21-xi-1825; same host, 3 ôd, 9 우, Ypanema, 29-ix-1819; same host,

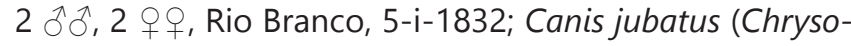
cyon brachyurus), 90 $\widehat{\partial}, 187$ 우, Registo de Rio Araguay, 21-x-1823 (Molin).

Status: Bunostomum trigonocephalus (Rudolphi, 1808) Railliet, 1902. 
Filaria trochili amethystini Molin, 1858

Filaria trochili amethystini, Molin, 1858b: 426.

Material: Trochilus amethystinus (Calliphlox amethystina), ad ventriculum, in Brasil.

Remarks: Species inquirendae in agreement with Molin (1858b); he found a single exemplar in very poor condition.

Status: The species should be considered nomen nudum.

\section{Ascaris truncata Zeder, 1800}

Ascaris hermaphrodita, Frölich, 1789: 151.

Fusaria truncata, Zeder, 1800: 105.

Ascaris truncata, Rudolphi, 1809: 193.

Ascaris truncata, Rudolphi, 1819: 657.

Ascaris (Ascaridia) truncata, Dujardin, 1845: 214.

Ascaris truncata, Diesing, 1851: 183.

Ascaridia hermaphrodita, Yorke \& Maplestone, 1926: 266.

Material: Psittaci n. 35 (Pyrrhura leucotis), Sapitiba, Februari 1818, in intestinis (Rudolphi). Psittacus guianensis (Aratinga leucophtalma), Aprili; Psittacus ararauna (Ara ararauna), Rio de Janeiro, Martio; Psittacus aracanga (Ara macao), Februari; Psittacus pulverulentus (Amazona farinosa), Januario; Psittacus purpureus (Touit purpuratus), Junio; Psittacus vinaceus (Amazona vinacea); Psittacus leucotis (Pyrrhura leucotis), Octobri; Psittacus phoenicurus (Pyrrhura molinae), Junio; Psittacus festivus (Amazona festiva), Forte do Rio Branco, Marti; intestino (Diesing).

Status: Synomym of Ascaridia hermaphrodita (Frölich, 1789) Yorke \& Maplestone, 1926.

\section{Dochmius tubaeformis (Zeder, 1800)}

Strongylus tubaeformis, Zeder, 1800: 73.

Dochmius tubaeformis, Dujardin, 1845: 279.

Dochmius tubaeformis, Diesing, 1851: 299.

Dochmius tubaeformis, Molin, 1861a: 487.

Ankylostomum tubaeforme, von Linstow, 1885: 237.

Material: Felis onça (Panthera onca), Matogrosso, Junio; Felis concolor (Puma concolor), Caiçara, Aprili, Junio, Novembri; Felis tigrina (Leopardus tigrinus), Felis mellivora (Herpailurus yagouaroundi), Februario, Aprili, Novembri (Diesing). $2 \hat{\jmath} \overline{0}, 2$ 우, Felis onça (Panthera onca), Matogrosso, 5-vi-1827; 188 $\widehat{\delta}, 355$ 웅, Puma concolor, as follows: 403 exemplars, Caiçara, 4-iv-1826; 38 exemplars, locality and date; 14 exemplar, Registro do Jauru, 19vi-1826; 31 exemplars, Caiçara, 19-xi-1825; 56 exemplars, same locality and date; $1 \hat{0}, 3$ 우, Felis tigrina (Leopardus tigrinus), Ypanema, 17-ix-1821; $13 \hat{\partial} \delta^{\lambda}, 10$ 우, same host and no more data; $54 \widehat{\partial} \widehat{\partial}, 60$ 우, Felis mellivora (Herpailurus yagouaroundi), as follows: 65 exemplars, Caiçara, 19-iv1826; 8 exemplars, same locality and date; 41 exemplars,

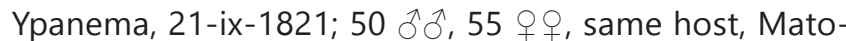
grosso, 22-ii-1929 (Molin).

Status: Ancylostoma tubaeformis (Zeder, 1800) von Linstow, 1885

Heterocheilus tunicatus Diesing, 1839

Lobocephalus heterolobus, Diesing, 1838: 189.

Heterocheilus tunicatus, Diesing, 1839: 230, Taf. XV, fig. 1-8.

Heterocheilus tunicatus, Diesing, 1851: 209.

Heterocheilus tunicatus, Drasche, 1883a: 108, Taf. IV,

fig. 1-5.

Material: Manatus exunguis (Trichechus inunguis), Borba, 13-i-1830, in ventriculo et in intestinis tenuibus.

Status: Heterocheilus tunicatus Diesing, 1839.

Filaria turdi olivascentis Molin, 1858

Filaria turdi olivascentis (palpebralis), Molin, 1858b: 423.

Material: Turdus olivacens (Coccycua minuta), Matogrosso, Octobri, sub membrana nictitante.

Remarks: Species inquirendae in agreement with Molin (1858b), who studied only 2 worms in poor condition.

Status: The species should be considered nomen nudum.

Physaloptera turgida Rudolphi, 1819

Physaloptera turgida, Rudolphi, 1819: 644.

Physaloptera turgida, Diesing, 1851: 233.

Physaloptera turgida, Molin, 1860d: 644.

Spiroptera turgida, Dujardin, 1845: 92.

Physaloptera turgida, Ortlepp, 1922: 1074

Spiroptera didelphis virginiana, Leidy, 1851: 155.

Turgida turgida, Travassos, 1920b: 54.

Material: Didelphis n. 7, intestinis (Rudolphi). Didel-

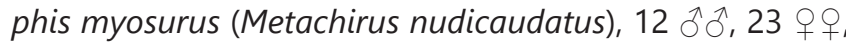
Ypanema, 25-i-1822 and 22-vii-1822; Didelphis cancrivora (Didelphis marsupialis), 3 ôे 9 , 9 우, Ypanema, 3-iii-1819, 8 exemplars, same host and locality, 10-xii-1821; Didelphis azarae (Didelphis aurita), 3 우우, Cuyaba, 20-vii-1824. In ventriculo.

Status: Turgida turgida (Rudolphi, 1819) Travassos, 1920.

Spiroptera umbellifera Molin, 1860

Spiroptera umbellifera, Molin, 1860a: 968.

Spiroptera umbellifera, Drasche, 1883b: 204, Taf. XII,

fig. 8 und 9.

Spiroptera umbellifera, Stossich, 1898: 107.

Sciadiocara umbellifera, Skrjabin, 1916: 520.

Material: Ibis rubra (Endocimus ruber), $1 \hat{0}, 1$, Praja de 
Cujutuba, 22-iii-1835; Totanus melanoleuca (Tringa melanoleuca), 4 우, Praja de Cujutuba, 1835, inter eorum tunicas ventriculi.

Status: Sciadiocara umbellifera (Molin, 1860) Skrjabin, 1916.

Ascaris uncinata Rudolphi, 1819

Ascaris uncinata, Rudolphi, 1819: 661.

Ascaris uncinata, Diesing, 1851: 147.

Paraspidodera uncinata, Travassos, 1914a: 139.

Paraspidodera uncinata, Hartwich, 1965: 124.

Material: Cavia aperea et Cavia pacae (Cuniculus paca), in intestino coeco.

Status: Paraspidodera uncinata (Rudolphi, 1819) Travassos, 1914.

Spiroptera uncinipenis Molin, 1860

Spiroptera uncinipenis, Molin, 1860a: 928.

Cheilospirura uncinipenis, Diesing, 1861: 684.

Cheilospirura uncinipenis, Drasche, 1883b: 211, Taf. XI,

fig. 6, 15, und 16; Taf. XIII, fig. 14.

Spiroptera uncinipenis, Stossich, 1898: 100.

Spirura uncinipenis, Railliet \& Henry, 1911c: 578.

Seurocyrnea uncinipenis, Chitwood \& Wehr, 1933: 296.

Sicarius unicinipenis, Freitas \& Lent, 1947b: 751.

Cyrnea (Procyrnea) unicinipenis, Chabaud, 1958: 490.

Habronema unicipenis, Yamaguti, 1961: 249.

Material: Rhea americana, $100 \hat{\partial} \hat{\partial}, 125 q q$, in Nas Lages, 4-iv-1823, and Cuyaba, 26-iii-1835, inter tunicas ventriculi.

Status: Procyrnea uncinipenis (Molin, 1860) n. comb.

Ascaris unguiculata Rudolphi, 1819

Ascaris unguiculata, Rudolphi, 1819: 653.

Ascaris unguiculata, Diesing, 1851: 151.

Aplectana unguiculata, Miranda, 1924: 46.

Oxysomatium unguiculatum, Harwood, 1930: 68.

Oxysomatium unguiculata, Hartwich, 1964c: 257.

Aplectana unguiculata, Baker, 1987: 60.

Material: Amphisbaenae n. 30, in intestinis (Rudolphi). Siphonops annulatus; Amphisbaena fuliginosa, Martio et Octobri; Glyptoderma vermiculare (Amphisbaena vermicularis), Julio; Lepidosternon microcephalus (Lepidosternon microcephalum), Novembri; Anops kingii, Junio, in intestinis crassis (Diesing).

Remarks: Species inquirenda in Baker (1987) but Hartwich (1964c) in his review of the types in the Zoological Museum of Berlin identify the species as Oxysomatium.

Status: Oxysomatium unguiculata (Rudolphi, 1819) Harwood, 1930.
Spiroptera unialata Molin, 1860

Spiroptera unialata, Molin, 1860a: 960.

Spiroptera unialata, Drasche, 1883b: 202, Taf. XI, fig. 8;

Taf. XIII, fig. 2.

Habronema unilateralis, Seurat, 1914d: 149.

Material: Rhamphastos erythrorhynchus (Rhamphastus tucanus), 2 ôे 11 q 1 , Barra do Rio Negro, 8-vi-1833, 4 $\$ \overline{0}, 23$ 우, same host and locality, in oesophago, in ventriculo et inter ejus tunicas; Ramphastus vitellinus, $2 \hat{\jmath} \widehat{\partial}, 4$ 오, Serra Arimani apud Rio Branco, in initio ventriculi et inter ejus tunicas; same host, $10 \hat{\jmath} \delta, 18+q+$, Barra do Rio Negro, 19-vi-1833, inter tunicas ventriculi.

Remarks: Drasche (1883b) states that this species is identical to Spiroptera unilateralis. That is followed by subsequent authors, but there are differences in the distribution of caudal papillae according to the figures of Drasche (fig. 2 vs. 12), including the presence of a precloacal papilla in Spiroptera unilateralis that is missing in Spiroptera unialata. Without reviewing the original material, the synonymy cannot be established with certainty.

Status: Procyrnea unialata (Molin, 1860) n. comb.

Spiroptera unilateralis Molin, 1860

Spiroptera unilateralis, Molin, 1860a: 945.

Spiroptera unilateralis, Drasche, 1883b: 200, Taf. XI, fig. 9; Taf. XIII, fig. 12.

Spiroptera unilateralis, Stossich, 1898: 103.

Habronema unilateralis, Seurat, 1914d: 151.

Cyrnea (Procyrnea) unilateralis, Chabaud, 1958: 490.

Material: Cephalopterus ornatus, $54 \hat{\partial} \hat{0}, 55 q+$, Egenho do Capo Gama, 23-viii-1823; 27 ôे $\hat{\jmath}, 49$ 우, Matogrosso,

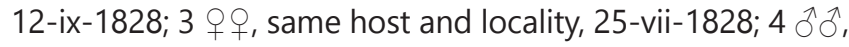
5 우, same host and locality, 10-ix-1828; $4 \hat{\jmath} \delta^{\lambda}, 3$ q $q$, Forte do Principe da Beira, 16-viii-1829, inter tunicas ventriculi.

Status: Procyrnea unilateralis (Molin, 1860) n. comb.

Ascaris valdemucronata Molin, 1860

Ascaris valdemucronata, Molin, 1860e: 339.

Ascaris valdemucronata, Diesing, 1861: 667.

Heterakis valdemucronata, Stossich, 1888: 293.

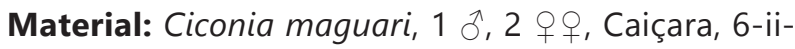
1826, crop and gizzard.

Status: Heterakis valdemucronata (Molin, 1860) Stossich, 1888.

Ascaris veligera Rudolphi, 1819

Ascaris veligera, Rudolphi, 1819: 656.

Ascaris veligera, Diesing, 1851: 173.

Dermatoxys veligera, Schneider, 1866: 123.

Material: Lepus brasiliensis (Sylvilagus brasiliensis), Aprili, in coeco. 
Status: Dermatoxys veligera (Rudolphi, 1819) Schneider, 1866.

Filaria verrucosa Molin, 1858

Filaria verrucosa, Molin, 1858b: 392.

Filaria dubia, Stossich, 1898: 85.

Serratospiculum verrucosum, Skrjabin, 1916: 546.

Serratospiculum verrucosum, Vicente et al., 1996: 95.

Material: Falco swainsonii (Gampsonyx swainsonii), $1+$ Matogrosso, Julio, inter musculos ad maxillam inferiorem.

Remarks: Stossich (1898) renamed this species as Filaria dubia without any reason.

Status: Serratospiculum verrucosum (Molin, 1858), Skrjabin, 1916.

\section{Heteracis verrucosa Molin, 1860}

Heteracis verrucosa, Molin, 1860e: 340.

Heteracis verrucosa, Diesing, 1861: 643.

Heteracis verrucosa, Drasche, 1882a: 120, Taf. VII, fig.

22.

Heterakis verrucosa, Hall, 1916: 105.

Material: Dasyprocta aguti, 5 $\hat{0}, 6$ + $q$, Caiçara, 23-i1826 , in ventriculo.

Status: Heterakis verrucosa (Molin, 1860) Hall, 1916.

\section{Spiroptera verrucosa Molin, 1860}

Spiroptera verrucosa, Molin, 1860a: 964.

Spiroptera verrucosa, Drasche, 1883b: 203, Taf. XI, fig.

18-20; Taf. XIII, fig. 1.

Pygarginema verrucosa, Kadenatsy, 1948: 151.

Pygarginema verrucosa, Vicente et al., 1998: 153.

Material: Cervus nambi (Mazama gouazoubira), 2 oे $\hat{\gamma}$, 1 , , Villa Maria, 16-ix-1825; Cervus dichotomus (Blastocerus dichotomus), $61 \hat{\jmath} \hat{\partial}, 100$ 우요, Registo do Rio Araguay, 22$\mathrm{x}-1823$, in eorum ventriculo.

Status: Pygarginema verrucosa (Molin, 1860) Kadenatsy, 1948.

Heteracis vesicularis (Frölich, 1791)

Ascaris vesicularis, Frölich, 1791: 85.

Ascaris vesicularis, Diesing, 1851: 148.

Heteracis vesicularis, Dujardin, 1845: 223.

Heteracis vesicularis, Molin 1861: 290.

Heterakis gallinae, Freeborn, 1923: 223.

Material: Phasianus gallus, Septembri, Cuyaba.

Status: Synonym of Heterakis gallinae (Schrank, 1788) Freeborn, 1923.

Spiroptera vulturis Molin, 1860

Spiroptera vulturis, Molin, 1860a: 976.

Spiroptera vulturis, Drasche, 1883b: 205, Taf. XI, fig. 26.
Filaria vulturis, Stossich, 1898: 51.

Material: Cathartes papa (Sarcorhamphus papa), 1 exemplar, Egenho do Cap Gama, 2-ix-1826, inter musculos maxillae inferioris.

Remarks: Species inquirenda in agreement with Molin (1860a), who examined only an exemplar in bad condition. According to the description, location, and the figure given by Drasche (1883b), it could be a kind of Thelazia or Oxyspirura (s.l.).

Status: Until the original material is reviewed, it should be considered incertae sedis.

Spiroptera vulvaria Molin, 1860

Spiroptera vulvaria, Molin, 1860a: 978.

Spiroptera vulvaria, Drasche, 1883b: 206.

Material: Momotus levaillanti (Momoti ruficapilli) (Momotus momota), 1 + , Porto do Rio Paraná, May; Icterus lacernulatus (Molothrus oryzivorus), 5 q $q$, Ypanema, 22-ix1822, inter eorum tunicas ventriculi.

Remarks: The name Icterus lacernulatus does not appear in the literature, but according to Molin (1860a) the vial with exemplars is marked as Orioli N. 49, which is in agreement with Pelzeln (1871). This number corresponds to Cassidix ater (= Molothrus oryzivorus), one of the two species of Icteridae collected in Ipanema in Septembri. Species inquirenda in agreement with Molin; he had only females.

Status: Drasche (1883a) pointed out "the lips look like Dispharagus," but no more references to the species are found in the literature, so until the original material has been reviewed, it should be considered incertae sedis.

\section{Spiroptera vulvoinflata Molin, 1860}

Spiroptera vulvoinflata, Molin, 1860a: 969.

Spiroptera vulvoinflata, Drasche, 1883b: 204, Taf. XII, fig. 15 und 16 (not 14 and 15); Taf. XIV, fig. 11.

Spiroptera vulvoinflata, Stossich, 1898: 122.

Serticeps vulvoinflatus, Railliet, 1916: 103.

Vigueira vulvoinflata, Mawson, 1968: 562.

Viguiera vulvoinflata, Vicente et al., 1996: 73.

Material: Trochilus ochropygus (Phaetornis pretrei), 2 ổ $3 \hat{1}, 2$ 우, Cuyaba, 8-x-1824, inter tunicas ventriculi.

Remarks: I agree with Drasche (1883b): "isn't Spiroptera would be Ancyracanthus."

Status: Viguiera vulvoinflata (Molin, 1860) Mawson, 1968.

Rudolphi (1819) and Diesing (1851) included some names in their monographs that correspond to specimens they could not identify and which some authors include as specific names, but all are nomina nuda. These are: 
Ardeae caeruleae Rudolphi, 1819: 711

Colubri Rudolphi, 1819: 712 = Nematoideum Colubri

Diesing, 1851: 337

Falconis albicollis Rudolphi, 1819: 711 = Nematoi-

deum Falconis albicollis Diesing, 1851: 333

Ranae Rudolphi, 1819: 713 = Nematoideum Ranae

Diesing, 1851: 338

Strigis Rudolphi, 1819: 711 = Nematoideum Strigis

Diesing, 1851: 334

Triglae Rudolphi, 1819: 713

Turdi Rudolphi, 1819: 711 = Nematoideum turdi

Diesing, 1851: 334

\section{Discusion}

A total of 323 names were used after studying Natterer's exemplars. From these, 163 are good species, 31 are as they were originally named, 117 have been transferred to another genus in the literature, and 15 were moved to another genus (n. comb.); 84 names are in doubtful position and the original specimens need to be studied, 49 are incertae sedis, 35 are species inquirendae, 76 names must be removed, 35 are synonyms of other species, and 41 are nomen nudum, most of them not described.

\section{Acknowledgments}

To Helmunt Satman from the Collection of Evertebrata Varia of the Naturhistorisches Museum in Vienna for allowing my stay in Vienna, the review of some exemplars collected by Naterrer, and his invaluable help with the original literature, and to Pedro Frade from the same institution for his information about this collection. To Birger Neuhaus from the Museum für Naturkunde in Berlin for help with the samples of Natterer in Berlin and to Sandra Miehlbradt from the same institution for sending me copies of letters and files from Natterer and Olfers. To Bernardo Urbani for his help with the names of monkeys and Gilson Rivas for help with the names of herps. To Scott L. Gardner for his invaluable work carefully reviewing this manuscript.

\section{References}

Abildgaard, P.Ch. 1795. Allgemeine Bemerkungen über Eingeweidewürmer. Skrivter af Naturhistorie-Selskabet Kjöbenhavn. 5: 26-64.

Alicata, J., McIntosh, A. 1933. Ascarops strongylina (Rud., 1819) the correct name for Arduenna strongylina (Rud.,
1819) Railliet et Henry, 1911 and Ascarops minuta Beneden, 1873. Journal of Parasitology 20: 62.

Anderson, R.A. 1959. Preliminary revision of the genus Diplotriaena Henry \& O'zoux, 1909 (Diplotriaenidae: Diplotriaeninae). Parassitologia 1: 195-307.

Anderson, R.C., Diaz-Ungría, C. 1959. Revisión preliminar de las especies de Thelazia Bosc (Spiruroidea Thelaziidae), parásitas de aves. Memoria de la Sociedad de Ciencias Naturales La Salle 52: 37-75.

Bain, O., Petit, G., Rosales-Loesener, L. 1986. Filaires de Singes sud-américains. Bulletin du Muséum national d'Histoire naturelle 4e. série 8 , section A, no. 3: 513-542.

Bain, O., Mutafchiev, Y., Junker, K., Guerrero, R., Martin, C., Lefoulon, E., Uni, S. 2015. Review of the genus Mansonella Faust, 1929 sensu lato (Nematoda: Onchocercidae), with descriptions of a new subgenus and a new subspecies. Zootaxa 3918: 151-193.

Baker, M.R. 1980. Revision of Old World species of the genus Aplectana Railliet \& Henry, 1916 (Nematoda, Cosmocercidae). Bulletin du Muséum national d'Histoire naturelle, 4e. série 8, section A, no. 2: 955-998.

Baker, M.R. 1987. Synopsis of the Nematoda parasitic in Amphibians and Reptiles. Memorial University of Newfoundland Occasional Papers in Biology 11: $1-325$.

Barreto, A.L. de B. 1919. On the Brazilian Species of the Subfamily Subulurinae Travassos, 1914. Memorias do Instituto Oswaldo Cruz 11: 10-70.

Baudin Laurencin, F. 1970. Oncophora melanocephala (Rud. 1819) n. comb., Nématode Camallanidae, parasite du thon albacore Neothunnus albacares. Bulletin du Muséum national d'Histoire naturelle 42: 984-988.

Baylis, H.A. 1920. On the classification of the Ascaridae. (i) Systematic value of certain characters of the alimentary canal. Parasitology 12: 253-264.

Baylis, H.A. 1932. Three notes on parasitic Nematodes. Annals and Magazine of Natural History, Series 10: 497-502.

Baylis, H.A. 1944. Notes on some parasitic Nematodes. Annals and Magazine of Natural History, Series 11: 793-804.

Baylis, H.A., Daubney, R. 1926. A synopsis of families and genera of Nematoda. The Oxford University Press, London. 277 pp.

Biocca, E. 1961a. Estudio di alcune specie di Nematodi descritti da Molin nel 1861, su esemplari ancora esistenti nella collezione elmintologica del Museo di Zoologia di Vienna. Nota 1. Ridescrizione di Ancylostoma (Ancylostoma) mucronatum (Molin, 1861). Parassitologia 3: 1-8.

Biocca, E. 1961b. Estudio di alcune specie di Nematodi descritti da Molin nel 1861, su esemplari ancora esistenti nella collezione elmintologica del Museo di Zoologia 
di Vienna. Nota 2. Osservazioni su Dochmius bidens e Dochmius maxillaris e redescrizione di Uncinaria bidens (Molin, 1961). Parassitologia 3: 9-21.

Blanchard, E. 1849. Recherches sur l'organisation de vers. Annales des Sociences Naturelles, Partie Zoologique 11: 106-202.

Boulenger, Ch.L. 1920. Filariid worms from mammals and birds in the Society's Gardens, 1914-1915. Proceedings of the Zoological Society of London 1920: 491-505.

Bouloenger, Ch.L. 1928. Report on a collection of parasitic nematodes mainly from Egypt. Part V. Filarioidea. Parasitology 20: 32-55.

Bremser, J.G. 1824. Icones helminthum systema Rudolphi entozoologicum illustrantes. Typis Antonii Strauss, Viennae. 12 pp.

Chabaud, A.G. 1957. Revue critique des nématodes du genre Quilonia Lane, 1914 et du genre Murshidia Lane, 1914. Annales de Parasitologie Humaine et Comparée 32: 98-131.

Chabaud, A.G. 1958. Essai de classification des nématodes Habronematinae. Annales de Parasitologie Humaine et Comparée 33: 445-508.

Chabaud, A.G. 1965. Ordre des Spirurida. In: Traite de Zoologie. Pierre Grasse, ed. Tome IV. Masson et Cie. Editors, Paris, pp. 1025-1151.

Chabaud, A.G., Bain, O. 1976. La lignée Dipetalonema. Nouvel essai de classification. Annales de Parasitologie Humaine et Comparée 51: 365-397.

Chabaud, A.G., Bain, O. 1981. Description de Spirobakerus weitzeli n. g., n. sp., et remarques sur les Nematodes Spirocercidae. Annales de Parasitologie Humaine et Comparée 56: 73-80.

Chabaud, A.G., Ball, G.H. 1964. Filaire cardiaque chez un Manchot des Galapagos Spheniscus mendiculus. Annales de Parasitologie Humaine et Comparée 39: 621-626.

Chabaud A.G., Petter, A.J. 1959. Essai de classification des Nematodes Acuariidae. Annales de Parasitologie Humaine et Comparée 34: 331-349.

Chandler, A.C. 1930. Specific characters in the genus Trichuris, with a description of a new species, Trichuris tenuis, from a camel. Journal of Parasitology 16: 198-206.

Chitwood, B.G. 1933. Note on a genus and species of nematode from Lynx canadensis. Journal of Parasitology 20: 63.

Chitwood, B.G., Wehr, E.E. 1933. The value of cephalic structures as characters in nematode classification, with special reference to the superfamily Spiruroidea. Zeitschrift für Parasitenkunde 7: 273-335.

Ciurea, J. 1912. Uber Spiroptera sexalata Molin aus dem Magen des Hausschweines. Zoologische Jahrbücher, Abteilung für Systematik, Geographie und Biologie der Tiere 32: 285-294.
Cram, E. 1927. Bird parasites of the nematode Suborders Strongylata, Ascaridata, and Spirurata. Bulletin of the United States National Museum 140: 1-465.

Creplin, F.C.H. 1825. Observationes de Entozois. Gryphiswaldiae. Sumtibus Mauritii Librarii, Gryphiswaldiae. 86 pp.

Creplin, F.C.H. 1829. Novae observationes de entozois. Apud Ferdinandum Dummlerum, Berlin. 134 pp.

Diaz-Ungría, C. 1963. Nématodes parasites, nouveaux ou interesants du Venezuela. Annales de Parasitologie Humaine et Comparée 38: 893-913.

Diaz-Ungría, C. 1964. Nematodes parásitos de aves venezolanas con descripción de una especie nueva. Boletin de la Sociedad Venezolana de Ciencias Naturales 107: 187-205.

Diesing, K.M. 1835. Tropisurus und Thysanosoma. Zwey neue Gattungen von Binnenwürmern (Entozoen) aus Brasilien. Medicisinche Jahrbücher des k. k. Öesterreichischen Staates 16: 83-116.

Diesing, K.M. 1838. Berichte der XV Versammlung deutscher Naturforscher und Aerzte, Prag. Seite 189.

Diesing, K.M. 1839 (1840). Neue Gattungen von Binnenwürmern nebst einem Nachträge zur Monographie der Amphistomen. Annalen des Wiener Museums der Naturgeschichte 2: 219-242.

Diesing, K.M. 1851. Systema Helminthum. Vol. 2. Apud Wilhelmum Braummüller. Vindobonae. 588 pp.

Diesing, K.M. 1855. Sechzehn Gattungen von Binnenwürmern und ihre Arten. Denkschriften der MathematischNaturwissenschaftliche Classe der Kaiserlichen Akademie der Wissenschaften Besonders Abgedruckt 9: 171-185.

Diesing, K.M. 1857. Sechzehn Arten von Nematoiden. Denkschriften der Mathematisch-Naturwissenschaftliche Classe der Kaiserlichen Akademie der Wissenschaften Besonders Abgedruckt 13: 6-26.

Diesing, K.M. 1861. Revision der Nematoden. Sitzungsberichte der Kaiserlichen Akademie der Wissenschaften, Mathematisch-Naturwissenschaftliche Classe 42: 595-736.

Drasche, R. von. 1882a. Revision der in der NematodenSammlung des k. k. Zool. Hofcabinetes befindlichen Original-Exemplare Diesing's und Molin's. Verhandlungen der kaiserlich-königlichen zoologisch-botanischen Gesellschaft in Wien 32: 117-138.

Drasche, R. von. 1882b. Helminthologische Notizen. Verhandlungen der kaiserlich-königlichen zoologischbotanischen Gesellschaft in Wien 32: 139-142.

Drasche, R. von. 1883a. Revision der in der NematodenSammlung des k. k. Zool. Hofcabinetes befindlichen Original-Exemplare Diesing's und Molin's. Fortsetzung. Verhandlungen der kaiserlich-königlichen zoologischbotanischen Gesellschaft in Wien 33: 107-118. 
Drasche, R. von. 1883b. Revision der in der NematodenSammlung des k. k. Zool. Hofcabinetes befindlichen Original-Exemplare Diesing's und Molin's. Fortsetzung II. Verhandlungen der kaiserlich-königlichen zoologischbotanischen Gesellschaft in Wien 33: 193-218.

Dujardin, F. 1845. Histoire naturelle des Helminthes ou vers intestinaux. Librairie Encyclopédique de Roret, Paris. 645 pp.

Esslinger, J.H. 1986. Redescription of Ochoterenella digiticauda Caballero, 1944 (Nematoda: Filarioidea) from the toad Bufo marinus, with a redefinition of the genus Ochoterenella Caballero, 1944. Proceedings of the Helminthological Society of Washington 53: 210-217.

Fitzinger, L.J. 1833. Frentag ben 28 Sept. Isis von Oken 26: 545-547.

Fitzinger, L.J. 1868. Geschichte des kais. kön. Hof-NaturalienCabinetes zu Wien. III. Abtheilung. Periode unter Kaiser Franz I. von Österreich von 1816 bis zu dessen Tode 1835. Sitzungsberichte der Kaiserlichen Akademie der Wissenschaften. Mathematisch-Naturwissenschaftliche Classe 58: 35-120.

Freeborn, S.B. 1923. The control of the suckered roundworms of poultry. The Cornell Veterinarian 13: 223-231.

Freitas, J.F.T. 1951. Alguns "strongyloidea" parasitos de Procyon cancrivorus (Cuv.) (Nematoda). Revista Brasileira de Biologia 11: 189-202.

Freitas, J.F.T., Lent, H. 1936. O gênero Monopetalonema Diesing, 1861 (Nematoda. Filarioidea). Memorias do Instituto Oswaldo Cruz 31: 747-757.

Freitas, J.F.T., Lent, H. 1937. Sobre Oswaldofilaria brevicaudata (Rodhain and Vuylsteke, 1937) n. comb. (Nematoda. Filarioidea). Memorias do Instituto Oswaldo Cruz 32: 439-442.

Freitas, J.F.T., Lent, H. 1939. Novo gênero de helmintos parasitos de roedores (Nematoda: Filarioidea). Boletim Biologico. 4: 14-18.

Freitas, J.F.T., Lent, H. 1947a. Revisão da subfamilia Deletrocephalinae, Railliet, 1916. Revista Brasileira de Biologia 7: 73-100.

Freitas, J.F.T., Lent, H. 1947b. Spiruroidea parasitos de $<$ Rheiformes > (Nematoda). Memorias do Instituto Oswaldo Cruz 45: 743-760.

Freitas, J.F.T., Rodrigues, H.O. 1964. Notula helmintológica: sobre o material original de "Dochmius bidens" Molin, 1861, atualmente existente no Museu de Viena (Nematoda). Atas da Sociedade Biologica de Rio de Janeiro 8: 48-50.

Frölich, J.A. 1789. Beschreibungen einiger neuen Eingeweidewürmer. Naturforscher 24: 101.

Frölich, J.A. 1791. Beiträge zur Naturgeschichte der Eingeweidewürmer. Naturforscher 25: 52-113.
Frölich, J.A. 1802. Beiträge zur Naturgeschichte der Eingeweidewürmer. Naturforscher 29: 5-96.

Gedoelst, L. 1919. Le genre Histiocephalus et les espèces qui y ont eté rapportées. Comptes Rendus Hebdomadaires de la Société de Biologie 82: 869-872.

Gendre, E. 1921 (1920). Sur une espèce nouvelle de d'Acuaria, parasite de Ptilopachys fusus Vieill. Extraits des Procès-Verbaux des Séances de la Société Linnéenne de Bordeaux 72: 34-39.

Gendre, E. 1922 (1921). Sur un helminthe peu connu Spiroptera conocephala Molin. Extraits des Procès-Verbaux des Séances de la Société Linnéenne de Bordeaux 73: 92-98.

Gendre, E. 1923 (1922). Sur quelques espèces d'Habronema parasites des oiseaux. Extraits des Procès-Verbaux des Séances de la Societé Linnéenne de Bordeaux. 74: 112-133.

Gendre, E. 1928. Nematodes. In: Joyeux, Ch., Gendre, E., Baer, J.G. Recherches sur les helminthes de I'Afrique occidentale française. Collection des Societe de Pathologie Exotic, Tome 2: 55-81. Paris. 120 pp.

Gibson, G.G. 1968. Species composition of the genus Streptocara Railliet et al., 1912 and the occurrence of these avian nematodes (Acuariidae) on the Canadian Pacific coast. Canadian Journal of Zoology 46: 629-645.

Giebel, C.G.A. 1857. Chr. L. Nitzsch's helminthologische Untersuchungen. Zeitschrift für die gesammten Naturwissenschaft 9: 264-269.

Gmelin, J.F. 1790. Systema naturae. Part 6, Vermes. Lipsiae. $3021 \mathrm{pp}$.

Goble, F.C., Kutz, H.L. 1945. The Genus Dispharynx (Nematoda: Acuariidae) in Galliform and Passeriform birds. Journal of Parasitology 31: 323-331.

Goeldi, E. 1896. Johannes von Natterer. Boletim do Mueu Paraense de Historia Natural e Ethnographia 1: 189-217.

Goeze, J.A.E. 1782. Versuch einer Naturgeschichte der Eingeweidewürmer thierischer Körper, Blankenburg. 471 pp.

Guerrero, R., Martin, C., Gardner, S.L., Bain, O. 2002. New and known species of Litomosoides (Nematoda: Filarioidea): Important adult and larval characters and taxonomic changes. Comparative Parasitology 69: 177-195.

Hall, M.C. 1916. Nematode parasites of mammals of the orders Rodentia, Lagomorpha, and Hyracoidea. Proceedings of the United States National Museum 20: 1-259.

Hall, M.C. 1921. Two new genera of nematodes, with a note on a neglected nematode structure. Proceedings of the United States Museum 59: 541-546.

Hartwich, G. 1957. Porrocaecum heteropterum (Diesing, 1851) nov. comb. (Nematoda: Ascaroidea), ein wenig bekannter 
Darmparasit aus Vögeln der Familie Threskiornithidae. Mitteilungen aus dem Zoologischen Museum in Berlin 33: 215-220.

Hartwich, G. 1964a. Revision der vogelparasitischen Nematoden Mitteleuropas. II. Die Gattung Contracaecum Railliet \& Henry, 1912 (Ascaridoidea). Mitteilungen aus dem Zoologischen Museum in Berlin 40: 15-53.

Hartwich, G. 1964b. Die Typen parasitischer Nematoden in der Helminthen-Sammlung des Zoologischen Museum in Berlin. I. Ascaridoidea. Mitteilungen aus dem Zoologischen Museum in Berlin 40: 55-65.

Hartwich, G. 1964c. Die Typen parasitischer Nematoden in der Helminthen-Sammlung des Zoologischen Museum in Berlin. II. Oxyuroidea, Atractoidea und Cosmodercoidea. Mitteilungen aus dem Zoologischen Museum in Berlin 40: 251-257.

Hartwich, G. 1965. Die Typen parasitischer Nematoden in der Helminthen-Sammlung des Zoologischen Museum in Berlin. III. Heterakoidea, Subuluroidea und Seuratoidea. Mitteilungen aus dem Zoologischen Museum in Berlin 41: 121-128.

Hartwich, G. 1966. Die Typen parasitischer Nematoden in der Helminthen-Sammlung des Zoologischen Museum in Berlin. IV. Filarioidea. Mitteilungen aus dem Zoologischen Museum in Berlin 42: 145-151.

Hartwich, G. 1970. Die Typen parasitischer Nematoden in der Helminthen-Sammlung des Zoologischen Museum in Berlin. V. Spiruroidea, Acuaroidea, Thelazioidea, Physalopteroidea, Spirura incertae sedis. Mitteilungen aus dem Zoologischen Museum in Berlin 46: 183-195.

Hartwich, G. 1971. Die Typen parasitischer Nematoden in der Helminthen-Sammlung des Zoologischen Museum in Berlin. VI. Strongyloidea, Trichostrongyloidea, Metastrongyloidea, Strongylida incertae sedis. Mitteilungen aus dem Zoologischen Museum in Berlin 47: 305-314.

Hartwich, G. 1972. Die Typen parasitischer Nematoden in der Helminthen-Sammlung des Zoologischen Museum in Berlin. VII. Rhabditoidea, Camallanoidea, Cucullanoidea, Dracunculoidea, Trichuroidea, Dioctophymatoidea, Mermithoidea und Nachträge zu Teil I-VI. Mitteilungen aus dem Zoologischen Museum in Berlin 48: 183-196.

Harwood, P.D. 1930. A new species of Oxysomatium (Nematoda) with some remarks on the genera Oxysomatium and Aplectana, and Observations on the life history. Journal of Parasitology 17: 61-73.

Hassall, H., Stiles, C.W. 1892. Strongylus rubidus, a new species of nematode, parasitic in pigs. Journal of Comparative Medicine and Veterinary Archives 13: 207-209.

Henry, A., O'zoux, L. 1909. La Filaire du Foudi. Bulletin de la Socieété de Pathologie Exotique 2: 544-547.
Hershkovitz, P. 1987. A History of the Recent Mammalogy of the Neotropical Region from 1942 to 1850 . Fieldiana, Zoology, new series 39: 11-98.

Inglis, W.G. 1958. The comparative anatomy of the subulurid head (Nematoda), with a consideration of its systematic importance. Proceedings of the Zoological Society of London 130: 577-604.

Inglis, W.G. 1965. The Nematodes parasitic in the gizzard of birds: a study in morphological convergence. Journal of Helminthology 39: 207-224.

Jägerskiöld, L.A. 1909: Zur kenntnis der NematodenGattungen Eustrongylides und Hystrichis. Nova Acta Reglae Societatis Scientiarum Upsaliensis. Ser IV. 2: 1-49.

Kadenatsy, A.N. 1948. [Pygarginema skrjabini n. g. n. sp. and Setaria kabargi n. sp. new nematodes of roe deer and musk deer]. Trudy Gelminth Laboratory Akademia Nauk SSSR 1: 149-153 (in Russian).

Leidy, J. 1850. Description of three Filariae. Proceedings of the Academy of Natural Sciences of Philadelphia 5: 117-118.

Leidy, J. 1851. Description of new species of entozoa. Proceedings of the Academy of Natural Sciences of Philadelphia 5: 155-156.

Leidy, J. 1856. A synopsis of entozoa and some of the other ecto-congeners observed by the author. Proceedings of the Academy of Natural Sciences of Philadelphia 8: 42-58.

Leidy, J. 1858. Contribution to helminthology. Proceedings of the Academy of Natural Sciences of Philadelphia 10: 25.

Lent, H., Freitas, J.F.T. 1942a. Contribuição ao conhecimento dos filarídeos de dasipodideos. Revista Brasileira de Biologia 2: 275-280.

Lent, H., Freitas, J.F.T. 1942b. Sobre Dipetalonema spiralis (Molin, 1860) (Nematoda, Filarioidea). Memorias do Instituto Oswaldo Cruz 37: 383-389.

Lent, H., Freitas, J.F.T. 1948. Una coleção de nematodeos parasitos de vertebrados, do Museu de historia natural de Montevideo. Memorias do Instituto Oswaldo Cruz 46: 1-71.

Leuckart, R. 1850. Helminthologische Notizen. Archiv für Naturgeschichte 16: 9-16.

Linnaeus, C. 1758. Systema Nature. Tome I, Holmiae, Impensis Direct, Laurentii Salvii. 847 pp.

López-Neyra, C.R. 1945. Estudios y revisión de la familia Subuluridae, con descripción de especies nuevas. Revista Ibérica de Parasitología 5: 271-329.

López-Neyra, C.R. 1951. Los Ascopsinae (Nematoda: Spirurata). Revista Ibérica de Parasitologia 11: 89-223.

López-Neyra, C.R. 1956. Revisión de la superfamilia Filarioidea (Weinland, 1858). Revista Ibérica de Parasitología 16: 3-225.

Mawson, P. 1968. Habronematinae (Nematoda: Spiruridae) from Australian birds. Parasitology 78: 745-767. 
Mehlis, E. 1831. Novae pbservationes de entozois. Isis von Oken 1831: 68-99.

Miranda, C. 1924. Alguns nematodeos do gênero Aplectana Railliet and Henry, 1916. Memorias do Instituto Oswaldo Cruz 17: 45-54.

Molin, R. 1858a. Spiroptera christoptera. Un nuovo verme intestinale del Tapiro americano. Verhandlungen der Kaiserlich-Königlichen Zoologischen-Botanischen Gesellschaft in Wien 8: 273-276.

Molin, R. 1858b. Versuch einer Monographie der Filarien. Sitzungsberichte der Kaiserlichen Akademie der Wissenschaften. Mathematisch-Naturwissenschaftliche Classe 28: 365-461.

Molin, R. 1858c. Prospectus helminthum, quae in prodromo faunae helminthologicae Venetiae continentur. Sitzungsberichte der Kaiserlichen Akademie der Wissenschaften. Mathematisch-Naturwissenschaftliche Classe 30: 127-158.

Molin, R. 1859a. Nuovi myzelmintha raccolti ed esaminati. Sitzungsberichte der Kaiserlichen Akademie der Wissenschaften. Mathematisch-Naturwissenschaftliche Classe 37: 818-854.

Molin, R. 1859b. Cephalocotylea e Nematoidea. Sitzungsberichte der Kaiserlichen Akademie der Wissenschaften. Mathematisch-Naturwissenschaftliche Classe 38: 7-38.

Molin, R. 1860a. Una monografia del genere Spiroptera. Sitzungsberichte der Kaiserlichen Akademie der Wissenschaften. Mathematisch-Naturwissenschaftliche Classe 38: 911-1005.

Molin, R. 1860b. Una monografia del genere Dispharagus. Sitzungsberichte der Kaiserlichen Akademie der Wissenschaften. Mathematisch-Naturwissenschaftliche Classe 39: 479-506.

Molin, R. 1860c. Una monografia del genere Histiocephalus. Sitzungsberichte der Kaiserlichen Akademie der Wissenschaften. Mathematisch-Naturwissenschaftliche Classe 39: 507-516.

Molin, R. 1860d. Una monografia del genere Physaloptera. Sitzungsberichte der Kaiserlichen Akademie der Wissenschaften. Mathematisch-Naturwissenschaftliche Classe 39: 637-672.

Molin, R. 1860e. Trenta specie di nematoidi. Sitzungsberichte der Kaiserlichen Akademie der Wissenschaften. Mathematisch-Naturwissenschaftliche Classe 40: 331-358.

Molin, R. 1861a. II sottordine degli Acrofalli ordinato scientificamente secondo I risultamenti delle indagini anatomiche ed embriogeniche. Memorie dell'i Reale Istituto Veneto di Scienze, Lettere ed Arti 9: 427-633.

Molin, R. 1861b. Podromus Faunae Helminthologicae Venetae. Adjectis disquisitionibus anatomicis et criticis. Denkschriften der Kaiserlichen Akademie der
Wissenschaften Mathematisch-Naturwissenschaftliche Classe 19: 189-338.

Moravec, F., Justine, J-L. 2020. Erection of Euterranova n. gen. and Neoterranova n. gen. (Nematoda, Anisakidae), with the description of E. dentiduplicata n. sp. and new records of two other anisakid nematodes from sharks off New Caledonia. Parasite 27, 58: 1-13. https://doi.org/10.1051/ parasite/2020053

Morgan, B.B. 1943. The Physalopterinae (Nematoda) of Aves. Transactions of the American Microscopical Society 62: 72-80.

Morgan, B.B. 1946. Host-parasite relationships and geographical distribution of the Physalopterinae (Nematoda). Transactions of the Wisconsin Academy of Sciences, Arts and Letters 38: 273-292.

Mozgovoy, A.A. 1951. [Ascaridata of mammals in the USSR (Anisakoidea)]. Trudy Gel'mintologicheskoy Laboratorii Akademii Nauk SSSR, 5, 14-22 (in Russian).

Mozgovoy, A.A. 1953. In: Skjabin, K.I., Shikhobalova, N.P., Mozgovoy, A.A. [Ascaridata of animals and man and the diseases caused by them]. Osnovy Nematodologii, Osnovy Nematodologii Tom 2. Publishing House of the USSR Academy of Sciences. Moscow, 616 pp. (in Russian).

Müller, O.F. 1780. Zoologicae Danicae seu animalorum Daniae et Norvegiae rariorium ac minus notorum icones. Havniae, 124 pp.

Neiva, A., Cunha, A.M., Travassos, L. 1914. Contribuições Parazitolojicas. Memorias do Instituto Oswaldo Cruz 6: 180-191.

Nitzsch, C.L. 1821. Ascaris. Allgemeine Encyclopadie der Wissensfchaftenf und Künste (Ersch. und Gruber). Arzneikunde-Azzolini, Leipzig. 6: 44-48.

Ortlepp, M.A. 1922. The Nematode Genus Physaloptera Rud. Proceedings of the Zoological Society of London 1922: 999-1107.

Owen. R. 1836. Anatomical descriptions of two species of Entozoa from the stomach of a tiger (Felis tigris Linn.), one of which forms a new genus of Nematoidea, Gnathostoma. Proceedings of the Zoological Society of London 4: 123-126.

Owen, R. 1843. Lectures on the comparative anatomy and physiology of the invertebrate animals. Delivered at the Royal College of Surgeons in 1843. London. 392 pp.

Papavero, N. 1971. Essays on the history of Neotropical Dipterology, with special reference to collectors (17501905). Vol. 1, Chapter V, "Collectors in Brasil." Museu de Zoologia da Universidade de Sao Paulo. Sao Paulo. Pp. 47-103.

Pelzeln, A. von. 1871. Zur Ornithologie Brasiliens. Resultate von Johann Natterers Reisen in den Jahren 1817 bis 1835. Druck und Verlag von A. Pichler's Witwe \& Sohn, Wien. $462 \mathrm{pp}$ 
Pelzeln, A. von. 1883. Brasilische Saugethiere. Resultate von Johann Natterers Reisen in den Jahren 1817 bis 1835. Verhandlungen der kaiserlich-königlichen ZoologischBotanischen Gesellschaft in Wien 33 (supl.): 1-136.

Petter, A.J. 1966. Équilibre des espèces dans les populations de nématodes parasites du colon des tortues terrestres. Mémoires du Muséum National de H'istoire Naturelle, nouvelle séries, Séries A, Zoologie 39: 1-252.

Pinto, R.M., Vicente, J.J., Noronha, D. 1996. Nematode Parasites of Brazilian Piciformes Birds: a General Survey with description of Procyrnea anterovulvata n. sp. (Habronematoidea, Habronematidae). Memorias do Instituto Oswaldo Cruz 91: 479-487.

Quentin, J.C. 1969. Essai de Classification des Nématodes Rictulaires. Mémoires du Muséum National de Histoire Naturelle, nouvelle séries, Séries A, Zoologie 54: 55-115.

Railliet, A. 1895. Traite de Zoologie Médicale et Agricole. 2nd ed. Asselin et Houzeau, Paris. 1303 pp.

Railliet, A. 1898. Rectification de la nomenclature d'après les travaux récents. Recuiel de Médicine Vétérinaire 75 : 171-174.

Railliet, A. 1902. Sur quelques sclerostomiens parasites des ruminants et des porcins. Comptes Rendus Hebdomadaires de la Société de Biologie 54: 107-110.

Railliet, A. 1916. La Famille des Thelaziidae. The Journal of Parasitology 2: 99-105.

Railliet, A., Henry, A. 1909a. Sur la classification des Strongylidae. I. Metastrongylinae. Comptes Rendus Hebdomadaires de la Société de Biologie 66: 86-88.

Railliet, A., Henry, A. 1909b. Sur la classification des Strongylidae. II. Ankylostominae. Comptes Rendus Hebdomadaires de la Société de Biologie 66: 168-171.

Railliet, A., Henry, A. 1909c. In: Henry, A. \& O'Zoux, L. 1909. La Filaire de Foudi. Bulletin Societe Pathologie Exotique 2: 544-547.

Railliet, A., Henry, A. 1910a. Les thélazies nématodes parasites de l'oeil. Comptes Rendus Hebdomadaires de la Société de Biologie 58: 213-216.

Railliet, A., Henry, A. 1910b. Les Onchocerques, Nématodes parasites du tissu conjonctif. Comptes Rendus Hebdomadaries des Société dé Biologie 58: 248-251.

Railliet, A., Henry, A. 1911a. Sur une Filaire péritonéale des Porcins. Bulletin de la Societé de Pathologie Exotique 4: 386-389.

Railliet, A., Henry, A. 1911b. Remarques au sujet des deux Notes de MM. Bauche et Bernard. Bulletin de la Societé de Pathologie Exotique 4: 485-488.

Railliet, A., Henry, A. 1911c. Les helminthes du nandou. Bulletin Societé Natationale d'Acclimatation 58: 573-582.

Railliet, A., Henry, A. 1912. Quelques Nématodes parasites des Reptiles. Bulletin de la Societé de Pathologie Exotique 5: 251-259.
Railliet, A., Henry, A. 1914. Essai de classification des "Heterakidae." IXe. Congrés International de Zoologie Tenu à Monaco. Pp. 674-682.

Railliet, A., Henry, A. 1915a. Sur les nématodes du genre Goezia Zeder. Bulletin de la Societé de Pathologie Exotique 8: 270-275.

Railliet, A., Henry, A. 1915b. Sur les nématodes du genre Camallanus Raill. et Henry, 1915 (Cucullanus auct., non Mueller, 1771). Bulletin de la Societé de Pathologie Exotique 8: 446-452.

Railliet, A., Henry, A. 1916. Nouvelles remarques sur les oxyuridés. Comptes Rendus Hebdomadaires de la Société de Biologie 79: 247-250.

Railliet, A., Henry, A., Sisoff, P. 1912. Sur les affinités des dispharages (Acuaria Bremser), nématodes parasites des oiseaux. Comptes Rendus Hebdomadaires de la Société de Biologie 73: 622-624.

Ransom, B.H: 1904. Manson's eye worm of chickens (Oxyspirura mansoni), with a general review of nematodes parasitic in eyes of birds. United States Deptment of Agriculture, Bureau of Animal Industry Bulletin 60: 1-54.

Rego, A.A. 1960. Redescrição de Artionema bidentata (Molin, 1858) n. comb. (Nematoda, Filarioidea). Memorias do Instituto Oswaldo Cruz 58: 149-156.

Ribeiro, D.J. 1941. Pesquisas helmintológicas realizadas no Estado do Pará. VIII. Camallanus amazonicus n. sp. parasito de Podocnemis expansus (Schw). Memorias do Instituto Oswaldo Cruz 35: 723-728.

Rodhain, P., Vuylsteke, C. 1937. Une filaire nouvelle d'Iguana tuberculata (Breinlia brevicaudata n. sp.). Annales de Parasitologie 15: 225-228.

Rudolphi, C.A. 1801. Beobachtungen über die Eingeweidewürmer. Archiv für Zoolologie und Zootomie 2: 1-65.

Rudolphi, C.A. 1802. Fortsetzung der Beobachtungen über die Eingeweidewürmer. Wiedemanns Archiv für Zoologie und Zootomie (Braunschweig) 2: 1-67.

Rudolphi, C.A. 1803. Neue Beobachtungen über die Eingeweidewürmer. Wiedemanns Archiv fur Zoologie und Zootomie (Braunschweig) 3: 1-32.

Rudolphi, C.A. 1808. Entozoorum sive vermium intestinalium historia naturalis. Vol. 1, Sumtibus Tabernae Librariae et Artium, Amstelaedami. 527 pp.

Rudolphi, C.A. 1809. Entozoorum sive vermium intestinalium historia naturalis. Vol. 2, part 1. Sumtibus Tabernae Librariae et Artium, Amstelaedami. 457 pp.

Rudolphi, C.A. 1819. Entozoorum synopsis cui accedunt Mantissa duplex et indices locupletissimi. Sumtibus Augusti Rücker. Berolini. 811 pp.

Ryzhikov, K.M. 1948. [Phylogenetic relationship of Nematodes of the Family Syngamidae and an attempt to 
reconstruct their systematics]. Docklady. Akademy Nauk SSSR, new series, 62: 733-736 (in Russian).

Saidov, I.S. 1953. [Revision of the Family Rhabdochonidae Skrjabin, 1946 and Subfamily Cyclozoninae Sobolev, 1949]. Rabot Gelminths, 75-Let Akad. Skrjabin. 662-635. Moscow (in Russian).

Sandground, J.H. 1938. On three species of filariid nematodes from sloths. Livro Jubilar Profesor Lauro Travassos, Brasil. pp. 421-428.

Sarmiento, L. 1959. Description of Paratractis hystrix (Diesing, 1851) gen. nov. (Nematoda: Atractidae) from Podocnemis dumeriliana. Journal of Parasitology 45: 65-67.

Schad, G.A. 1962. Studies on the Genus Kalicephalus (Nematoda: Diaphanocephalidae). II. A Taxonomic Revision of the Genus Kalicephalus Molin, 1861. Canadian Journal of Zoology 40: 1035-1165.

Schmutzer, K. 2011. Der Liebe zur Naturgeschichte halber. Johann Natterers Reisen in Brasilien 1817-1836. Veroffentlichungen der Kommission fur Geschichte der Naturwissenschaften, Mathematik und Medizin Nr. 64 Osterreichische Akademie der Wissenschaften, Vienna. 360 pp.

Schneider, A. F. 1866. Monographie der Nematoden. Druck und Verlag von Georg Reimeil, Berlin. 357 pp.

Schrank, F.P. 1788. Verzeichniss der bisher hinlänglich bekannten Eingeweidewürmer, nebst einer. Abhandlung über ihre Anverwandtschaften. München 116 pp.

Schröckinger-Neudenberg, J. von. 1855. Zur Erinnerung an einen österreichischen Naturforscher. Verhandlungen des zoologisch-botanischen Vereins in Wien 5: 727-732.

Seurat, L.G. 1914a. Sur I'Habronema (Spiroptera) leptoptera (Rud.). Comptes Rendus Hebdomadaires de la Société de Biologie 76: 21-23.

Seurat, L.G. 1914b. Sur un nouveau parasite de la perdix rouge. Comptes Rendus Hebdomadaires de la Société de Biologie 76: 390-393.

Seurat, L.G. 1914c. Sur un nouveau Spiroptére des Rapaces. Comptes Rendus Hebdomadaires de la Société de Biologie. 76: 427-430.

Seurat, L.G. 1914d. Sur un nouveau parasite du Perenoptére. Bulletin de la Societé d'Histoire Naturelle de Afrique du Nord 6: 149-153.

Seurat, L.G. 1915. Sur les premières stades évolutifs des spiropteres. Comptes Rendus Hebdomadaires de la Société de Biologie 78: 561-565.

Seurat, L.G. 1916. Sur les oxyures des mammifères. Comptes Rendus Hebdomadaires de la Société de Biologie 79: 64-68.

Siebold, C.Th. 1848. Lehrbuch der vergleichenden Anatomie der Wirbellosen Thiere. Verlag von Veit \& Comp. Berlin. 679 pp.

Skrjabin, K.I. 1915. [filaria in birds of Turkestan. 1.
Serratospiculum turkestanicum n. g., n. sp. 2. Diplotriaena tricuspis Fedtsch., 1894]. Vestnik Obshei Veterinar 4: 131138 (in Russian).

Skrjabin, K.I. 1916. Nématodes des oiseaux du Turkestan russe. Annals Museum de Zoologie de l'Academie Imperial des Sciences Petrograd. 20: 457-557.

Skrjabin, K.I. 1917. Sur quelques nématodes des oiseaux de la Russie. Parasitology 9: 460-481.

Skrjabin, K.I., Sobolev, A.A. 1964. [Spirurata of animals and man and the diseases caused by them. Part 4. Thelazioidea. Principles of Nematology, ed. K.I. Skrjabin, Vol. XII]. Izdatelsvo Nauka, Moscow. 334 pp.

Skrjabin, K.I., Sobolev, A.A., Ivashkin, B.M. 1967. [Spirurata of animals and man and the diseases caused by them. Part 4. Thelazioidea. Principles of Nematology, ed. K.I. Skrjabin, Vol. XVI]. Izdatelsvo Nauka, Moscow. 624 pp.

Smith, A. 1908. Synopsis of studies in Metazoan Parasitology in Momanes Laboratory of Pathology, University of Pennsylvania. University of Pennsylvania Medical Bulletin 20: 262-282.

Sonin, M.D. 1968. [Filariata of animals and man and the diseases caused by them. Part 2. Diplotriaenoidea. Principles of Nematology, ed. K.I. Skrjabin, Vol. XXI]. Izdatelsvo Nauka, Moscow. 388 pp. (in Russian).

Sprent, J.F.A. 1958. Observations on the development of Toxocara canis (Werner, 1782) in the dog. Parasitology 48: 184-209.

Sprent, J.F.A. 1959. The life history and development of Toxascaris leonina (von Linstow, 1902) in the dog and cat. Parasitology 49: 330-371.

Sprent, J.F.A. 1968. Notes on Ascaris and Toxascaris, with a definition of Baylisascaris gen. nov. Parasitology 58: 185-198.

Sprent, J.F.A. 1979a. Ascaridoid nematodes of amphibians and reptiles: Multicaecum and Brevimulticaecum. Journal of Helminthology 53: 91-116.

Sprent, J.F.A. 1979b. Ascaridoid nematodes of amphibians and reptiles: Terranova. Journal of Helminthology 53: 265-282.

Stiles, C.W. 1901. A discussion of certain questions of nomenclature as applied to parasites. Zoologische Jahrbücher, Abteilung für Systematik, Geographie und Biologie der Tiere 15: 157-208.

Stiles, C.W., Hassall, A. 1920. Index-Catalogue of Medical and Veterinary Zoology: Roundworms. United States Public Health Service, Hygienic Laboratory, Bulletin No. 114. 886 pp.

Stossich, M. 1888. II genere Heterakis Dujardin. Glasnika Hrvatakoga Naravoslovnoga Drutzva, Zagreb 2: 277-301.

Stossich, C.W. 1889. II genere Physaloptera Rudolphi. Bollettino della Società adriatica di scienze naturali in Trieste 11: 36-59. 
Stossich, M. 1891a. Elminti Veneti raccolti dal Dr. Alessandro Conte de Ninni. Bollettino della Società adriatica di scienze naturali in Trieste 12: 50-56.

Stossich, M. 1891b. Il genere Dispharagus Dujardin. Bollettino della Società adriatica di scienze naturali in Trieste 13: 81-108.

Stossich, M. 1896. II genere Ascaris Linné. Lavoro Monografico. Bollettino della Società adriatica di scienze naturali in Trieste 17: 9-119.

Stossich, M. 1898. Filarie e spiroptere Lavoro Monografico. Bollettino della Società adriatica di scienze naturali in Trieste 18: 13-162.

Stossich, M. 1899. Strongylidae. Lavoro Monografico. Bollettino della Società adriatica di scienze naturali in Trieste 19: 55-152.

Stossich, M. 1901. Contributo allo studio degli elminti. Bollettino della Società adriatica di scienze naturali in Trieste. 20: 1-9.

Tollitt, M.E. 1987. Case 2604. Dioctophyme Collet-Meygret, 1802 (Nematoda): proposed confirmation of spelling (ClOMS Case No. 7). Bulletin of Zoological Nomenclature 44: 237-239.

Travassos, L. 1914a. Contributions to the study of Brazilian Helmithology. III. A new genus of the family Heterakidas Railliet and Henry. Memorias do Instituto Oswaldo Cruz 6: 137-142.

Travassos, L. 1914b. Contribuções para o conhecimento da fauna helmintologica brazileira. V. Sobre as especies brasileiras do genero Tetrameres Creplin, 1846. Memorias do Instituto Oswaldo Cruz 6: 150-162.

Travassos, L. 1915. Contribuções para o conhecimento da fauna helmintologica brazileira. 3. Sobre as especies brasileiras do genero Capillaria Zeder, 1800. Memorias do Instituto Oswaldo Cruz 7: 146-172.

Travassos, L. 1917a. Trichostrongylinas brazileiras. Brazil Medico 31: 73

Travassos, L. 1917b. Alguns helminthos da colleção do Instituto Bacteriologico de S. Paulo. Brazil Medico 31: 99-100.

Travassos, L. 1918a. Trichostrongylidae brazileiras. Revista da Sociedade de Sciencias do Rio do Janeiro 3: 191-205.

Travassos, L. 1918b. Contribução para o conhocimento da fauna helminthologica Brazileira. VII. Especies brazileiras do genero Thelazia Bosc, 1819. Revista do Museo Paulista 10: 215-230.

Travassos, L. 1920a. Contributions à l'etude de la fauna helminthologique du Brasil. IX. Les espèces du genre Spinicauda n. g. Memorias do Instituto Oswaldo Cruz 12 : 41-50.

Travassos, L. 1920b. Contribução á Sistematica dos Physalopterinae. Revista de Sciencias 4: 54-55.

Travassos, L. 1921. XIII. Essai monographique sur la famille
Trichostrongylidae Leiper, 1909. Memorias do Instituto Oswaldo Cruz 13: 1-135.

Travassos, L. 1923. Informações sobre a fauna helmintologica de Matto Grosso. Oxyuroidea-Heterakoidea. Folha Medica 4: 35-37.

Travassos, L. 1926. Ascaris retusa (Rud., 1819) Boletim Biologico 4: 87-93.

Travassos, L. 1927. Uma nova Capillaria parasita de peixes de agua doce; Capillaria sentinosa n. sp. Boletim Biologico 1: 215-217.

Travassos, L. 1929a. Filaridés des batraciens du Brésil. Comptes Rendus Hebdomadaires de la Société de Biologie 100: 967-968.

Travassos, L. 1929b. Contribucão ao conhecimento des Strongyloidea parasitos do Tapirus americanus. Memorias do Instituto Oswaldo Cruz 22: 135-144.

Travassos, L. 1931. Pesquizas helminthologicas realizadas em Hamburgo. IX. Ensaio monographico da familia Cosmocercidae Trav., 1925. Memorias do Instituto Oswaldo Cruz 25: 237-298.

Travassos, L. 1933. Sobre os filarideos dos crocodilos sulamericanos. Memorias do Instituto Oswaldo Cruz 27: 159-164.

Travassos, L. 1937. Revisão da familia Trichostrongylidae Leiper, 1912. Monographias do Instituto Oswaldo Cruz 1. $512 \mathrm{pp}$.

Travassos, L., Freitas, J.F.T., Lent, H. 1939. Relatório da excursão scientífica do Instituto Oswaldo Cruz, realizada na zona da Estrada de Ferro Noroeste do Brasil em outubro de 1938. II. Pesquisas helmintológicas. Boletim Biologico 4: 221-249.

Vanzolini, P.E. 1993. As viagens de Johann Natterer no Brasil, 1817-1835. Papéis Avulsos de Zoologia 38: 17-60.

Vaz, C., Pereira, C. 1929. Arduenninae de Bradypus tridactylus L. Boletim Biologico 15: 1-15.

Vevers, G.M. 1922. On the Parasitic Nematoda collected from Mammalian Hosts which died in the Gardens of the Zoological Society of London during the Years 1919-21; with a Description of Three New Genera and Three New Species. Proceedings of the Zoological Society, London 1922: 901-919.

Vicente, J.J., Rodrigues, H.O., Gomes, D.C., Pinto, R.M. 1991. Nematóides do Brasil. 2a Parte: Nematóides de Anfíbios. Revista brasileira de Zoologia 7: 549-626.

Vicente, J.J., Rodrigues, H.O., Gomes, D.C., Pinto, R.M. 1993. Nematóides do Brasil. III. Nematóides des Répteis. 10: 19-168.

Vicente, J.J., Rodrigues, H.O., Gomes, D.C., Pinto, R.M. 1995 (1996). Nematóides do Brasil. Parte IV: Nematóides de Aves. Revista Brasileira de Zoologia. 12 (supl. 1): 1-273.

Vicente, J.J., Rodrigues, H.O., Gomes, D.C., Pinto, R.M. 1997 (1998). Nematóides do Brasil. Parte V: Nematóides de 
Mamíferos. Revista Brasileira de Zoologia 14 (supl. 1): 1-452.

von Linstow, O. 1879. Helminthologische Untersuchungen. Jahreshefte des Vereins fur vaterländische Naturkunde in Württemberg 35: 313-342.

von Linstow, O. 1885. Beobachtungen an bekannten und neuen Nematoden und Trematoden. Archiv für Naturgeschichte 51: 235-255.

Walton, A.C. 1927. A revision of the nematodes of the Leidy collections. Proceedings of the Academy of Natural Sciences of Philadelphia 79: 49-163.

Weber, W.A.F. 1955. The filarial parasites of primates: A review. II. Loa, Protofilaria and Parlitomosa, with notes on incompletaly identified adult and larval forms. Annals of Tropical Mededicine and Parasitology 49: 235-249.

Wedl, C. 1856. Ueber die Mundwerkzeuge von Nematoden. Sitzungsberichte der Kaiserlichen Akademie der Wissenschaften. Mathematisch-Naturwissenschaftliche Classe 19: 33-69.

Werner, P.C.F. 1782. Vermium intestinalium brevis expositionis continuatio. Lipsiae. 28 pp.

Wong, P.L., Anderson, R.C., Bartlett, C.M. 1986. Revision of the genus Syncuaria Gilbert, 1927 (Nematoda: Acuarioidea). Canadian Journal of Zoology 64: 1186-1196. https://doi.org/10.1139/z86-177
Wong, P.L., Lankester, M.W. 1985. Revision of the genus Ancyracanthopsis Diesing, 1861 and description of a new genus Molinacuaria n. gen. (Nematoda: Acuarioidea). Canadian Journal of Zoology 63: 1556-1564. https://doi. org/10.1139/z85-231

Yamaguti, S. 1961. Systema Helminthum. Vol. 3, parts 1 and 2. Interscience Publishers, New York. 1261 pp.

Yeh, K.-S. 1957. On a filarial parasite, Deraiophoronema freitaslenti n. sp., from the giant anteater, Myrmecophaga tridactyla from British Guiana, and a proposed reclassification of Dipetalonema and related genera. Parasitology 47: 196-205.

Yorke, W., Maplestone, P.A. 1926. The Nematode Parasites of Vertebrates. J. \& A. Churchill, London. 536 pp.

Zeder, J.G.H. 1800. Erster Nachtrag zur Naturgeschichte der Eingeweidewürmer mit Zufässen und Anmerkungen herausgegeben. Leipzig. 320 pp.

Zeder, J.G.H. 1803. Anleitung zur Naturgeschichte der Eingeweidewürmer. Bamberg. 432 pp. 